\title{
Black hole uniqueness theorems in higher dimensional spacetimes
}

\author{
Stefan Hollands ${ }^{1 *}$ and Akihiro Ishibashi ${ }^{2 \dagger}$ \\ ${ }^{1}$ School of Mathematics, Cardiff University, \\ Cardiff, United Kingdom \\ ${ }^{2}$ Department of Physics, Kinki University, \\ Higashi-Osaka, Japan
}

August 31, 2012

\begin{abstract}
We review uniqueness theorems as well as other general results about higher dimensional black hole spacetimes. This includes in particular theorems about the topology of higher dimensional spacetimes, theorems about their symmetries (rigidity theorem), and the classification of supersymmetric black holes. We outline the basic ideas underlying the proofs of these statements, and we also indicate ways to generalize some of these results to more general contexts, such as more complicated theories.
\end{abstract}

\section{Introduction}

The prediction of the existence of black holes by general relativity is one of the most intriguing ones in all of theoretical physics, and there is now compelling evidence that such objects might indeed exist in Nature. Apart from thus describing, in all likelihood, real astrophysical objects, black holes are also viewed as a theoretical laboratory for ideas about the, not yet completely understood, laws of quantum gravity. These connections arise in particular through the analogy between the "laws of black hole mechanics", and the laws of thermodynamics [6]. They strongly suggest that black holes should have some sort of (quantum) statistical mechanical description analogous to, say, the (quantum) statistical mechanical description of a gas comprised of atoms, see e.g. [131] for a review.

\footnotetext{
*HollandsS@cardiff.ac.uk

†akihiro@phys.kindai.ac.jp
} 
Both in the astrophysical context, as well as in the context of quantum gravity, the striking uniqueness, also referred to as no hair property, of stationary black hole solutions plays an important role: All (regular) stationary, asymptotically flat solutions of the EinsteinMaxwell equations in $D=4$ dimensions are uniquely determined by their mass, angular momentum, and electric charge, and are in fact given by the Kerr-Newman family of solutions. In the astrophysical context, this result is important because one expects that real physical black hole systems, while not being stationary as long as they form, will eventually settle down and thus eventually become stationary. Assuming this to be true, one can then restrict attention to the explicitly known Kerr-Newman family of solutions. Moreover, one can study small non-stationary perturbations of such a system within the well-understood framework of linear perturbation theory, or by Post-Newtonian methods, possibly corrected by radiation-reaction effects, thus eliminating, in a large dynamical range, the need to solve numerically the full set of non-linear Einstein-Maxwell equations. In the quantum gravity context, one also relies in many approaches on the fact that the stationary black hole solutions are functions of only a few conserved charges. While many classical properties of black holes are probably lost in a full quantum regime, one still expects that such charges can be defined as quantum charge operators, that an ensemble of physical quantum states in the Hilbert space, characterized by a definite values of these parameters, can be identified, and that quantities such as entropy can be assigned to such an ensemble.

It is clearly of interest to ask whether a version of the black hole uniqueness theorem still holds under more general assumptions, for example (a) in the presence of other Einsteinmatter fields systems such as Einstein-Yang-Mills, Einstein-scalar fields, Einstein-Euler, Einstein-Vlasov, Einstein-Skyrmions, etc. and (b) in more than four spacetime dimensions.

a) Inclusion of matter fields: As a rule of thumb, if the matter sector without coupling to gravity already contains soliton-like static or stationary solution, then one has to expect that the corresponding theory coupled to Einstein-gravity has hairy black holes. This is, in some sense, not really surprising, so it is generally more interesting to study the uniqueness of systems in which the matter sector does not have soliton solutions in and of itself. An interesting example of this type is Einstein-Yang-Mills theory. That theory does not have any soliton like solutions without coupling to gravity, and neither has the vacuum Einstein theory. Nevertheless, for suitable gauge groups $\left(\pi_{2}(G) \neq 0\right)$, hairy black holes were found, to the surprise of many researches, in the coupled theory. While the solutions that were originally found turned out to be unstable, there do exist stable hairy black holes in other Einstein-matter theories, and furthermore, there are even hairy black holes which do not have any kind of symmetry other than time-translations. Thus, for several Einstein-matter systems, the uniqueness theorems definitely fail to hold. For a detailed discussion of black hole uniqueness in $D=4$, including many references, see the review [66, 21].

b) Higher dimensions: The possibility that there could exist more than the four macroscopically large, observed, dimensions has been pursued almost from the beginning of general relativity theory. One of the main attractive features of this idea, common to most 
approaches, is that matter fields naturally "arise from geometry". In string theory, extra dimensions appear naturally from constraints imposed by the quantum nature of strings propagating in a higher dimensional target space (usually $D=26$, or $D=10$ ). Particular values for the number of dimensions also arise in supergravity theories due to the constraining nature of the supersymmetry algebra. In practice, string theories are often analyzed within the supergravity approximation (especially in the context of the AdS-CFT correspondence and its variants), hence by some sort of classical gravity theory in higher dimensional spacetime.

From a physical viewpoint one has to explain why, if extra dimensions indeed exist, we do not directly observe them. In the standard Kaluza-Klein approach, the extra dimensions are assumed to be extremely small (e.g. tori, or other compact manifolds with special properties, having small volume). Then, any fields with non-trivial dependence in the extra dimensions effectively have a very large mass (small deBroglie wavelength), and thus would decay into lighter fields in a quantum field theoretic description. There are, however, also other theoretical scenarios in which the extra dimensions are not small, but macroscopically large. In this type of model, it is assumed that the four dimensions which we observe effectively constitute some timelike submanifold ("brane") in a higher dimensional spacetime, to which the relevant matter fields (standard model) are confined in some way. In the classical context, one means by this usually that the contribution to the stress tensor by observable matter fields is localized on, or very near to, the brane. Apart from studying black hole solutions with boundary conditions at infinity corresponding to the Kaluza-Klein respectively brane setup, one can also study higher dimensional black holes with standard asymptotically flat boundary conditions, i.e. which are asymptotic to $D$-dimensional Minkowski space (or e.g. $D$-dimensional (Anti-)deSitter spacetime, where appropriate). The latter are motivated particularly in the context of holography, wherein a higher dimensional gravity theory with AdS-boundary describes a quantized field theory on the boundary. Asymptotically flat boundary conditions are not well-motivated physically in higher dimensions. However, they can serve as a clean setup to isolate new phenomena of higher dimensional gravity theories.

In this review article, we will concentrate on uniqueness theorems in higher dimensional gravity theories, (b). Of course, because higher dimensional theories can always be reduced to lower dimensional ones with additional matter fields via the Kaluza-Klein reduction process, (b) includes also many models in (a). Not surprisingly therefore, much less is known about the uniqueness theory of higher dimensional black holes than in 4 dimensions, in much the same way as much less is known about general Einstein-matter systems compared to the vacuum theory in 4 dimensions. Generally speaking, one might expect to be in either of the following three, qualitatively different, situations:

1. ("Best possible case") This would mean that all black hole solutions in the given theory (e.g. vacuum general relativity in $D$ dimensions, or including various special matter fields) would be specified uniquely by a finite set of "conserved charges" that are calculable as surface integrals over a large $(D-2)$-dimensional surface near infinity. For 
example, in pure Einstein-gravity, these would be the conserved ADM-type mass, and the ADM-angular momenta (or more restrictively even, as in $D=4$, the angular momenta corresponding to rotational Killing fields). In theories involving also matter fields such as Maxwell fields, these would include additionally e.g. electric/magnetictype charges etc. It is clear that we cannot to be in this situation for general matter fields, because it is known even in 4 dimensions that black hole uniqueness theorem in this form does not hold e.g. for the Einstein-Yang-Mills system, see (a). It is also clear that we cannot expect to be in this situation even for pure Einstein-gravity in $D$ dimensions, because solutions of 4-dimensional Einstein- $S U(N)$-Yang-Mills theory are solutions of this theory via the Kaluza-Klein reduction process e.g. if the $D$ dimensional manifold is $\mathscr{M} \times S U(N)$, with $\mathscr{M}$ the 4-dimensional spacetime manifold. The latter solutions are not asymptotically flat from the point of view of the higher dimensional spacetime (they are asymptotically Kaluza-Klein, see below), so one might hope that black hole uniqueness in the sense of the "best possible case" might hold e.g. in pure Einstein-gravity in $D$ dimensions, if we restrict to stationary black holes that are asymptotically flat in the $D$-dimensional sense (i.e., asymptotic to $D$-dimensional Minkowski spacetime). However, this-to the surprise of many researchers-also turned out to be false when Emparan and Reall [32] discovered 5-dimensional regular, stationary asymptotically flat black "rings" which had the same mass and angular momenta as the previously known Myers-Perry solutions [107], but were not isometric to these and even had a different horizon topology (explicit forms of many higher dimensional black hole solutions are given e.g. in the review [33]). Thus, we definitely cannot be be in the "best possible case".

2. ("Intermediate situation") Since higher dimensional black holes cannot be uniquely specified by their conserved charges (ADM-mass, ADM-angular momenta, electric charge $(\mathrm{s}), \ldots$ ) alone, one might hope that they might be uniquely specified if one adds to these parameters further ones that somehow specify internal degrees of freedom of the stationary black holes that cannot be read off from the asymptotics at infinity via the conserved charges alone. A hint that this expectation might not be unreasonable comes from other systems of PDE's of geometrical nature admitting soliton-like solutions, but not containing the gravitational field. For example, it is well-known, that the space of self-dual solutions to the Yang-Mills equations $F=\star F$, modulo gauge transformations, on a sufficiently generic, compact, Riemannian 4-manifold $\mathscr{M}$ is itself a manifold $X_{k}$ (see e.g. [5]), whose dimension is given by $\operatorname{dim} X_{k}=8 k-3$ [for $S U(2)$ ], where $k=c_{2}(E)$ is the second Chern number of the associated bundle $E \rightarrow \mathscr{M}$, interpreted as the instanton number. The local coordinates on $X_{k}$ have an interpretation in terms of the relative position, orientation, and size, of the instantons represented by the gauge field. Similarly, the space of $k$-centered BPS monopole solutions modulo gauge transformation on $\mathbb{R}^{3}$ with gauge group $S U(N)$ is known to be a finite-dimensional manifold, see e.g. [4]. The local coordinates of $X_{k}$ again have an 
interpretation in terms of the position, orientation, velocity and magnetic charges of the monopoles. Furthermore, in both examples, the dimension of the space of solutions modulo gauge transformations is related to the index of some differential operator. By analogy with these non-linear systems of PDE's, one may hope that the space of, say black hole solutions with $k$ components of the event horizon of given topology modulo gauge transformations, may be given by a manifold $X_{k}$ (or maybe more generally, a space with certain singularities) whose local coordinates describe the relative position, size, orientation etc. of the black hole horizons, and possibly other data encoding the topology/shape of the ambient manifold surrounding the black holes. One may further hope that the dimension of the space $X_{k}$ may be related to the index of some operator associated with the linearized Einstein equations. The present knowledge of black hole solutions in pure Einstein-gravity does not contradict this scenario, and it seems plausible that this should be the typical situation for supersymmetric solutions (in supergravities). However, there are no general results.

From the classical viewpoint, proving scenario 2) would be no less satisfactory than 1). However, in the context of quantum gravity, the situation is less clear. While the conserved charges should retain their role also in quantum theory as operators whose eigenvalues are the charge quantum numbers of the corresponding quantum states, (as they do in ordinary quantum field theory), the role of the further "internal parameters" in scenario 2) at the quantum level is much less clear.

3. ("Worst case scenario") The most general possibility is that the space of stationary black hole solutions, with, say, a fixed number of horizon components, is not finite dimensional in nature, i.e. that there are "free function(s) worth" of solutions with given asymptotic charges. In this case, there seems to be little point in trying to prove a "uniqueness theorem".

In summary, while it is known that we are not in case 1), it is unknown whether we are in case 2) or 3), even for asymptotically flat, stationary vacuum black holes in $D>4$ dimensions.

Related to the question of black hole uniqueness is that of stability. If a black hole is unstable, then one may expect that the endpoint of the evolution of an instability will be a new kind of black hole-assuming of course that the system settles down at all. Furthermore, stability analyses can in principle detect the existence of new stationary black holes which are perturbatively close to a given family of black holes. To understand the stability issue for a given family of stationary black holes, one may, for simplicity, start by looking at the linearized field equations around that background (although one should emphasize that linear stability does not necessarily imply stability against small finite perturbations). Unfortunately, already in the simplest cases such as a Schwarzschild black hole, the analysis of these equations is still rather involved [135]. (See also [90, 80]. For a more modern treatment of the scalar version of the stability problem based on the method of "energy norms", see 
e.g. [26].) In the four dimensional Kerr case, there is available the Teukolsky formalism [128] which effectively reduces the full set of coupled perturbation equations to that of a single complex variable. Furthermore, that equation can be studied via separation of variables techniques, and this analysis shows that the Kerr black hole is stable "mode-by-mode". Unfortunately, there is no known formalism of comparable power neither in higher dimensions, or in 4 dimensions with more general matter fields. Progress has been made (see [29] and refs. therein) in higher dimensions for certain kinds of stationary black holes which are characterized by a wide separation of scales between various parameters characterizing the black hole, e.g. "long-thin" (highly prolate), or "flat thin" (highly oblate) horizons, partly based on numerical methods. Some of these black holes have thereby been determined to be unstable, and the nature of the instability is qualitatively similar to that known before for the 5-dimensional black string [56]. However, there is no complete picture. There are also other approaches to the black hole stability problem based on variational principles related to "local Penrose inequality", or on the notion of thermodynamic stability, see [40, 76]. Unfortunately, also these methods do not give a complete picture of stability of standard families of higher dimensional black holes.

Linearized analyses can also detect whether a new family of stationary black holes is "branching off" from a given family. Indeed, if a given black hole admits a stationary (nongauge) perturbation, which is not tangent to the original family of solutions, then this is evidence-although not proof-for a new family of solutions. This has also been carried out in some examples [29], and evidence has been found for the existence of a 70-parameter family of new stationary solutions in $D=9$ dimensions branching off from Myers-Perry black holes in a certain parameter range [28]. In particular, evidence has been found for families of solutions with a very small number of Killing vectors (i.e. with the minimum possible symmetry compatible with the rigidity theorem, see below). Evidence in the same direction has also come from an entirely different approximation scheme, wherein the black object is treated as some sort of extended test-object, in much the same way as one can treat small objects as point-particles. This so-called "blackfold approach" [34] likewise indicates the existence of, yet unknown, asymptotically flat solutions with a very small number of Killing vectors. Thus, it appears from a variety of viewpoints that the manifold of black hole solutions in higher dimensions is largely unknown.

The plan of this article is as follows: We first outline, schematically, the proof of the black hole uniqueness theorem for Einstein-Maxwell theory in 4 dimensions. We then critically review to what extent the steps in this analysis generalize to higher dimensional theories, outlining the method of proof of the key intermediate theorems, which are also interesting in their own right as general structure theorems for higher dimensional black holes. We then present black hole uniqueness theorems in higher dimensions in the static, resp. stationary, case in higher dimensions. In the first case a rather complete picture emerges (in particular for supersymmetric solutions in certain supergravity theories), but in the second case, results are currently available only if one imposes more symmetry on the solution than what one has the right to expect in view of the rigidity theorem and also the numerical evidence. We 
will mostly restrict the discussion to either vacuum general relativity or simple extensions thereof, but we will try to indicate in what situation the techniques can be generalized to more general theories. Also, we will restrict attention mostly to asymptotically flat, or asymptotically Kaluza-Klein boundary conditions at infinity, and we will (except in the discussion of supersymmetric solutions) usually assume that the black hole is not extremal.

\section{General structure of uniqueness proof and history in $D=4$}

For comparison with the higher dimensional case, we first give an outline of the proof that the Kerr-Newman black holes [87, 109] are the only stationary, asymptotically flat black hole solutions in Einstein-Maxwell theory in $D=4$. The action is

$$
S=\int_{\mathscr{M}} \frac{1}{2} R \star_{4} 1+F \wedge \star_{4} F
$$

Here, $\mathscr{M}$ is a four dimensional spacetime manifold, with Lorentzian metric ${ }^{1} g$ and the field strength is $F=\mathrm{d} A$. It is assumed that $g, A$ satisfy the asymptotic conditions

$$
g_{\mu \nu}=\eta_{\mu \nu}+O\left(r^{-1}\right), \quad A_{\mu}=O\left(r^{-1}\right)
$$

in a Cartesian coordinate system $x^{\mu}$ (and an appropriate gauge for $A$ ) that is defined in the asymptotic region of $\mathscr{M}$. In particular, the asymptotic region is required to be diffeomorphic to $\mathbb{R}$ times $\mathbb{R}^{3} \backslash B_{r}^{3}$, with $B_{r}^{3}$ a sufficiently large ball of radius $r$ with respect to the radial Cartesian coordinate $r=\sqrt{x_{1}^{2}+x_{2}^{2}+x_{3}^{2}}$. As usual, it is also required that derivatives fall off by a correspondingly higher power. The metric and gauge field are stationary in the sense that there exists a vector field $t$ on $\mathscr{M}$ which is equal to $\partial / \partial x^{0}$ in the asymptotic region, such that

$$
\mathscr{L}_{t} g=0=\mathscr{L}_{t} A
$$

The asymptotic conditions allow one to construct the asymptotic null infinities $\mathscr{I}^{ \pm}$of the spacetime (see the conformal diagram of Schwarzschild below ${ }^{2}$ ), and the black hole may then be defined rigorously as the complement of the past of future null infinity,

$$
\mathrm{BH}=\mathscr{M} \backslash I^{-}\left(\mathscr{I}^{+}\right), \quad \mathrm{WH}=\mathscr{M} \backslash I^{+}\left(\mathscr{I}^{-}\right),
$$

and similarly the white hole. $I^{ \pm}$denote the chronological future/past of a set. It is of course understood that $\mathrm{BH}$ region should not be empty. As we will never be concerned with the interior of the black hole, we will normally use the symbol $\mathscr{M}$ for the exterior of

\footnotetext{
${ }^{1}$ Except in sec. 4.3 , our signature convention is $(-++\ldots)$.

${ }^{2}$ The extended Schwarzschild spacetime has two asymptotic ends, but in the rest of this paper we will restrict attention to one given asymptotic end of the spacetime under consideration.
} 
the black and white hole, also called "domain of outer communication" (for a more precise definition see sec. 3.1). It corresponds to the blue region in the figure. $\mathscr{M}$ has an inner boundary, $\partial \mathscr{M}=\mathscr{H}$, comprised of the union of the future and past horizon $\mathscr{H}^{ \pm}$. We wish to understand what solutions $(A, g)$-subject possibly to further technical assumptions such as analyticity/global causal structure - there can be.

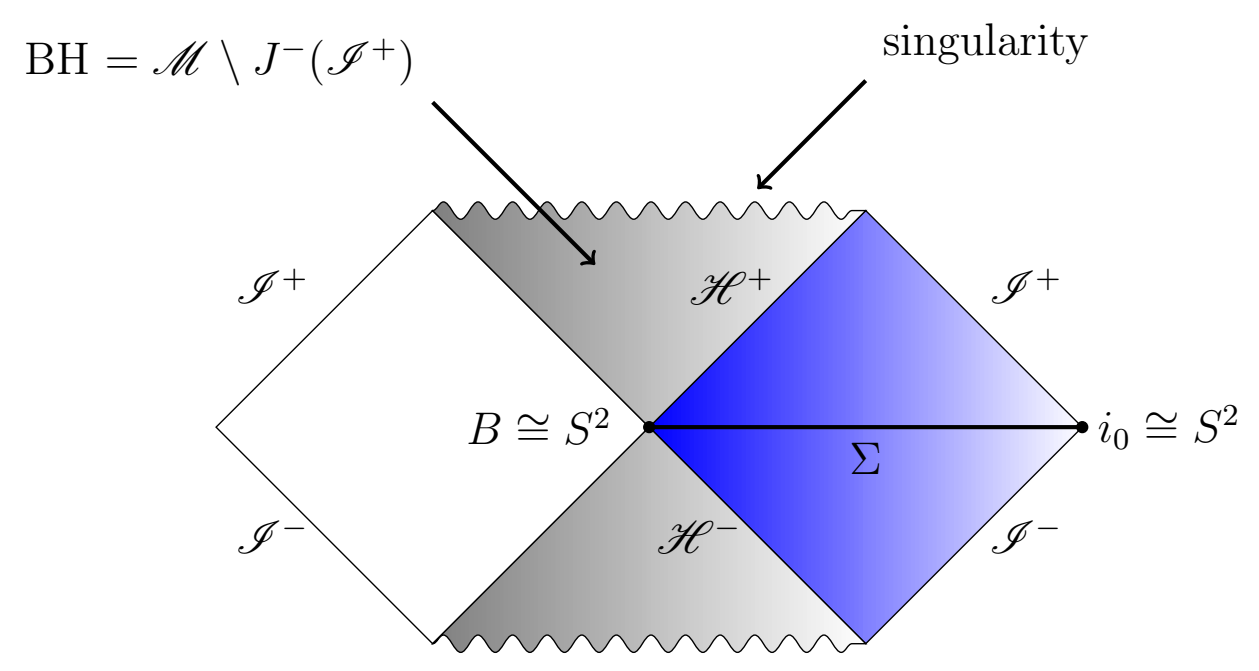

The analysis proceeds in the following steps (we restrict ourselves for simplicity to nonextremal black holes, $\kappa>0$, see eq. (7)):

1. Using the geometric nature of the horizon $\mathscr{H}$, and the field equations, one shows $[62,63]$ that each horizon cross section is topologically a sphere, $B \cong S^{2}$. This is called the "topology theorem". In particular $\mathscr{H}^{ \pm} \cong \mathbb{R} \times S^{2}$.

2. Now consider the restriction of $t$ to the horizon, $\mathscr{H}=\mathscr{H}^{+} \cup \mathscr{H}^{-}$. Then we can be in either of the following cases: (a) The horizon is non-rotating, in the sense that $t$ is null on $\mathscr{H}$, i.e. tangent to the null-generators. Then the "staticity theorem" of [126] implies that the spacetime is not only stationary but even static. This means that there is a foliation by Cauchy surfaces $\Sigma(\tau)$ of $\mathscr{M}$ intersecting the bifurcation surface $B$ such that $t$ is orthogonal to each $\Sigma(\tau)$. In fact, we may chose $\tau$ as one of the coordinates, and then, identifying $\Sigma(\tau)$ with $\Sigma$ along the orbits of $t$, we can write $\mathscr{M}=\mathbb{R} \times \Sigma$,

$$
g=-N^{2} \mathrm{~d} \tau^{2}+h, \quad A=\phi \mathrm{d} \tau, \quad t=\frac{\partial}{\partial \tau}
$$

where $h$ is a Riemannian metric on $\Sigma, N>0$ a smooth function (lapse), and $\phi$ is the electro static potential, all independent of $\tau$.

(b) On the other hand, if $t$ is not tangent to the generators on $\mathscr{H}$, then the horizon is rotating. Using the topology theorem $B \cong S^{2}$, and that a vector field on $S^{2}$ necessarily 
vanishes somewhere, one can then establish the "rigidity theorem" [63], under the assumption of analyticity of the spacetime. This theorem states that there exists a second Killing field $\psi$ on $(\mathscr{M}, g, A)$ (i.e., both $g$ and $A$ are Lie-derived by $\psi$ ) commuting with $t$ such that the flow generated by $\psi$ has closed orbits with period, say, $2 \pi$, and such that

$$
K=t+\Omega \psi,
$$

is tangent to the null-generators of the horizon. In particular, the horizon is a "Killing horizon," whose surface gravity $\kappa>0$, defined through

$$
\nabla_{K} K=\kappa K
$$

can then be shown ${ }^{3}$ to be constant on $\mathscr{H}$ [130]. The constant $\Omega$ is interpreted "angular velocity" of the horizon.

At this stage, the argument branches off, depending on whether one is in the non-rotating (i.e. static) case (a), or the rotating case (b).

3a) Static case: In the static case, there are now several arguments that the solution under consideration must be the (regular, non-degenerate) Reissner-Nordstrom solution, characterized uniquely by its mass $M$ and electric charge $Q$. Traditionally, one proceeds via Israel's theorem [84] which states that the solution must be rotationally symmetric, i.e. invariant under $O(3)$. The rest is a straightforward integration of Einstein's equation subject to staticity and $O(3)$-symmetry, as is done in Schwarzschild. A more modern version is given in $[124]^{4}$ and $[13,100]$. The method of the last paper is particularly elegant and consists of the following steps, where we restrict for simplicity the discussion to the vacuum case, $A=0$. One starts by doubling the spatial slice $(\Sigma, h)$ across the horizon cross section, gluing a copy $\bar{\Sigma}$ onto $\Sigma$ along $B$. One then performs a conformal rescaling from $h$ to $\tilde{h}=\Omega^{2} h$ on the doubled spacetime $\tilde{\Sigma}=\Sigma \cup \bar{\Sigma}$, with $\Omega$ chosen in such a way that (a), the scalar curvature $\tilde{R}$ of $\tilde{h}$ is non-negative, (b) such that $\Omega \rightarrow 1$ near the spatial infinity $i_{0}$ of $\Sigma$, and (c) such that $\Omega \rightarrow 0$ near the spatial infinity $\bar{i}_{0}$ of $\bar{\Sigma}$, in such a way that $\bar{\Sigma}$ is effectively compactified, or "capped off". (d) The mass $\tilde{M}$ of $(\tilde{\Sigma}, \tilde{h})$ is zero. Then the positive mass theorem is applied to $(\tilde{\Sigma}, \tilde{h})$, implying that $\tilde{h}$ is in fact the flat Euclidean metric on $\tilde{\Sigma}=\mathbb{R}^{3}$. The rest of the proof then proceeds by showing that $\Omega$ must be precisely so that $\Omega^{-2} \delta=h$ is equal to the spatial part of the Schwarzschild metric in isotropic coordinates, from which it then easily follows that the spacetime itself is Schwarzschild. We will give a more detailed account of this argument, taken from [52] and valid also for higher dimensions and various matter fields, in sec. 3.8.

\footnotetext{
${ }^{3}$ The proof of this statement only requires that the Einstein equation holds with a stress tensor satisfying the dominant energy condition.

${ }^{4}$ Note that his argument contained a gap.
} 
3b) Rotating case: Here one proceeds as follows (again we restrict attention for simplicity to $A=0)$. First, using Einstein's equation, one shows that the metric on $\mathscr{M}$ can be written globally in Weyl-Papapetrou form, meaning that globally, coordinates $r>$ $0, z, \tau$, together with a $2 \pi$-periodic coordinate $\varphi$ can be chosen, in such a way that

$$
g=-\frac{r^{2} \mathrm{~d} \tau^{2}}{f}+\mathrm{e}^{-\nu}\left(\mathrm{d} r^{2}+\mathrm{d} z^{2}\right)+f(\mathrm{~d} \varphi+w \mathrm{~d} \tau)(\mathrm{d} \varphi+w \mathrm{~d} \tau), \quad \psi=\frac{\partial}{\partial \varphi}, \quad t=\frac{\partial}{\partial \tau} .
$$

The remaining components of the metric, $f, \nu, w$ are functions of $r>0, z$ only, and they obey equations that follow from the original Einstein equations. Assume now that there were two solutions with equal values of the mass $M$ and angular momentum $J$ relative to the Killing field, defined as

$$
J=\frac{1}{8 \pi} \int_{\infty} \star_{4} \mathrm{~d} \psi
$$

where the integral is over a large sphere in the asymptotic region. Then, one derives, using the Weyl-Papapetrou form that both metrics must satisfy, a partial differential equation in $r, z$ for a quantity measuring the "difference" between non-trivial metric components of the two metrics. The particular form of this identity, called "Robinson identity" [119] allows one to prove that this "difference" actually has to be zero, and hence that the metrics actually have to coincide. It was originally not clear how to generalize the Robinson identity to the case of Einstein-Maxwell theory in 4 dimensions, but this problem was later solved by [101] and [12]. Their work in particular showed that the Robinson identity can be understood from the point of view of certain nonlinear sigma-models. It is this viewpoint that proves useful also for other theories, and in higher dimensions, and we will review it in some more detail below in sec. 3.5.

\section{Higher dimensions}

In this section, we will critically investigate the various steps described above in 4 dimensional Einstein Maxwell theory, and see to what extent they can be generalized to higher dimensions. We will then explain what kinds of uniqueness theorems are presently available. For simplicity, we will mostly restrict to vacuum general relativity, and to non-extremal black holes [i.e. non-vanishing surface gravity, see eq. (17)], but we will also comment on more general cases, mostly in sec. 4.

\subsection{Asymptotic conditions}

Let $(\mathscr{M}, g)$ be a $D$-dimensional, stationary black hole spacetime $D \geq 4$. The asymptotically timelike Killing field is called $t=t^{\mu} \partial_{\mu}$, so $\mathscr{L}_{t} g=0$. Depending on the theory under consideration, or the nature of the solutions that one is interested, one may wish to impose 
different asymptotic conditions on the metric and corresponding conditions on the matter fields, if those are present in the theory. Standard asymptotic conditions on the metric are:

1. Asymptotically flat boundary conditions: The metric approaches $D$-dimensional Minkowski spacetime $\mathbb{R}^{D-1,1}$ at large distances as in eq. (2), with $O\left(r^{-1}\right)$ replaced by $O\left(r^{-(D-3)}\right)$, or at late advanced/retarded time ("null infinity"). Note that the asymptotic symmetry group of an asymptotically flat spacetime is $S O(D-1,1) \times \mathbb{R}^{D}$ (semi-direct product). In particular, the number $N$ of commuting Killing fields with circular orbits must correspond to a subgroup $U(1)^{N} \subset S O(D-1,1)$ of the Cartan subalgebra. Hence,

$$
N \leq\left\lceil\frac{D-1}{2}\right\rceil .
$$

Here $\lceil x\rceil$ is by definition the largest integer $n \leq x$.

2. If the theory has a negative cosmological constant, or scalar fields with a minimum of the potential that effectively provides this, then asymptotically Anti-deSitter (AdS) boundary conditions are appropriate, see e.g. [67], or many other references. These boundary conditions can be viewed as saying that a suitably conformally rescaled spacetime $\Omega^{2} g$ has a timelike conformal boundary. Similarly, if the theory has a positive cosmological constant, then it is reasonable to consider asymptotically deSitter "boundary" conditions. In that case, one has a spacelike conformal boundary. We will not discuss here either type of theory, although they are of considerable interest and various exact black hole solutions have been found. However, especially in the AdS case, recent investigations indicate that the manifold of black holes may be very complicated $[27,9]$.

3. Asymptotically Kaluza-Klein (KK) boundary conditions (see below): The spacetime is asymptotically $\mathbb{R}^{s, 1} \times Y^{D-s-1}$, where $Y^{D-s-1}$ is a compact Riemannian manifold $(D-s-$ 1 extra-dimensions). Alternatively, one can consider the asymptotics $A d S_{s+1} \times Y^{D-s-1}$ or $d S_{s+1} \times Y^{D-s-1}$. Again, if we have $N$ commuting circular Killing fields, then $N$ is restricted by the number of circular symmetries that $\mathbb{R}^{s, 1} \times Y^{D-s-1}$ has, and similarly in the $d S$ and $A d S$-cases. The maximum $N=D-3$ can be achieved if $s=1,2,3,4$, and $Y^{s-D-1} \cong \mathbb{T}^{D-s-1}$. We will exclusively deal with this case in this review.

4. Other variants of the above asymptotic conditions can also be considered. For example, one might replace the asymptotically flat condition by "locally asymptotically flat". This means that the spacetime is not asymptotically $\mathbb{R}^{D-1,1}$, but instead $\mathbb{R}^{D-1,1} / \Gamma$, where $\Gamma \subset O(D-1)$ is some discrete subgroup of the spatial rotations. For example, in $D=5$, we could take a cyclic subgroup $\Gamma=\mathbb{Z}_{p} \subset O(4)$. Then the large spheres $S^{3}$ near spatial infinity are replaced by a quotient $S^{3} / \mathbb{Z}_{p}$. Of course, one can consider also other discrete subgroups, KK-quotients, etc. Black hole spacetimes of this nature have been given e.g. by [18]. 
5. One may also study "braneworld boundary conditions"; such black holes have recently been found numerically $[38,39]$. We will not consider them here.

Asymptotically KK-boundary conditions are in more detail as follows: We assume that a subset of $\mathscr{M}$ is diffeomorphic to the cartesian product of $\mathbb{R}^{s}$ with a ball removed - corresponding to the asymptotic region of the large spatial dimensions - and $\mathbb{R} \times \mathbb{T}^{D-s-1}$ —corresponding to the time-direction and small dimensions. We will refer to this region as the asymptotic region and call it $\mathscr{M}_{\infty}$. The metric is required to behave in this region like

$$
g=-\mathrm{d} \tau^{2}+\sum_{i=1}^{s} \mathrm{~d} x_{i}^{2}+\sum_{i=1}^{D-s-1} \mathrm{~d} \varphi_{i}^{2}+O\left(R^{-s+2}\right),
$$

where $O\left(R^{-\alpha}\right)$ stands for metric components that drop off at least as fast as $R^{-\alpha}$ in the radial coordinate $R=\sqrt{x_{1}^{2}+\ldots+x_{s}^{2}}$, with $k$-th derivatives in the coordinates $x_{1}, \ldots, x_{s}$ dropping off at least as fast as $R^{-\alpha-k}$. These terms are also required to be independent of the coordinate $\tau$, which together with $x_{i}$ forms the standard cartesian coordinates on $\mathbb{R}^{s, 1}$. The remaining coordinates $\varphi_{i}$ are $2 \pi$-periodic and parameterize the torus $\mathbb{T}^{D-s-1}$. The timelike Killing field is assumed to be equal to $\partial / \partial \tau$ in $\mathscr{M}_{\infty}$. We call spacetimes satisfying these properties asymptotically Kaluza-Klein spacetimes ${ }^{5}$.

The domain of outer communication is defined by

$$
\langle\langle\mathscr{M}\rangle\rangle=I^{+}\left(\mathscr{M}_{\infty}\right) \cap I^{-}\left(\mathscr{M}_{\infty}\right),
$$

where $I^{ \pm}$denote the chronological past/future of a set. The black hole region $B$ is defined as the complement in $\mathscr{M}$ of the causal past of the asymptotic region, and its boundary $\partial B=\mathscr{H}$ is called the (future) event horizon. Since we will never be concerned with the interior of the black hole, we will simply write $\mathscr{M}$ again for the domain of outer communication.

In this paper, we also sometimes assume the existence of $D-3$ further linearly independent Killing fields, $\psi_{1}, \ldots, \psi_{D-3}$, so that the total number of Killing fields is equal to the number of spacetime dimensions minus two. These are required to mutually commute, to commute with $t$, and to have periodic orbits. The Killing fields $\psi_{i}$ are referred to as "axial" by analogy to the four-dimensional case, even though their zero-sets are generically higher dimensional surfaces rather than "axis" in $D>4$, see the discussion below in sec. 3.4. We also assume that, in the asymptotic region $\mathscr{M}_{\infty}$, the action of the axial symmetries is given by the standard rotations in the cartesian product of flat Minkowski spacetime $\mathbb{R}^{s, 1}$ times the standard flat torus $\mathbb{T}^{D-s-1}$. In other words, $\psi_{i}=\partial / \partial \varphi_{i}$ or $^{6} \psi_{j}=x_{2 j-1} \partial_{x_{2 j}}-x_{2 j} \partial_{x_{2 j-1}}$ for $j=1, \ldots,\lceil s / 2\rceil$ in $M_{\infty}$. The group of isometries is hence $G=\mathbb{R} \times K$, where $\mathbb{R}$ corresponds to the flow of $\tau$, and where $K=\mathbb{T}^{D-3}$ corresponds to the commuting flows of the axial Killing fields.

\footnotetext{
${ }^{5}$ For the axisymmetric spacetimes considered in this paper, we will derive below a stronger asymptotic expansion, see [74]

${ }^{6}$ The notation $\lceil x\rceil$ means the largest integer $n$ such that $n \leq x$.
} 
Unfortunately, in order to make many of the arguments in the following sections in a consistent way, one has to make certain further technical assumptions about the global nature of $(\mathscr{M}, g)$ and the action of the symmetries. Our assumptions are in parallel to those made by Chruściel and Costa in their study [20] of 4-dimensional stationary black holes. The requirements are (a) that $\mathscr{M}$ contains an acausal, spacelike, connected hypersurface $\Sigma$ asymptotic to the $\tau=0$ surface in the asymptotic region $\mathscr{M}_{\infty}$, whose closure has as its boundary $\partial \Sigma=B$ a cross section of the horizon. We always assume $B$ to be compact but we allow for multiple components. (b) We assume that the orbits of $t$ are complete. (c) We assume that the horizon is non-degenerate. (d) We assume that $\mathscr{M}$ is globally hyperbolic. We will also occasionally assume (e) that the spacetime, the metric, and the group action are analytic, rather than only smooth.

\subsection{Rigidity theorem}

The rigidity theorem is not only a key ingredient in the black hole uniqueness theorems, but also important on its own right. This is because it shows that every stationary black hole horizon is, in fact, a Killing horizon, i.e. that there is a Killing field $K$ tangent to the generators of $\mathscr{H}$ commuting with $t$, or coinciding with it. That in turn implies [130] the constancy of the surface gravity, which otherwise would not even be defined. Because the constancy of the surface gravity is physically interpreted as the constancy of the temperature of the black hole (zeroth law of black hole mechanics), this result is of fundamental physical importance, and it is also the basis for the other laws of black hole mechanics. The precise statement of the rigidity theorem $[72,103]$ is as follows:

Theorem 1. ("Rigidity theorem") Let $(\mathscr{M}, g)$ be an asymptotically flat, analytic stationary black hole solution to the vacuum Einstein equations. Assume further that the event horizon, $\mathscr{H}$, of the black hole is analytic and is topologically $\mathbb{R} \times B$, with $B$ compact and connected, and that the average surface gravity $\langle\kappa\rangle \neq 0$ (see eq. (17) below). Then there exists a Killing field $K$, defined in a region that covers $\mathscr{H}$ and the entire domain of outer communication, such that $K$ is normal to the horizon and $K$ commutes with $t$.

The rigidity theorem has the following consequence [72, 103]:

Theorem 2. Under the same assumptions made in Theorem 3.1 above, if $t$ is not tangent to the generators of $\mathscr{H}$, then there exist mutually commuting Killing fields $\psi_{1}, \ldots, \psi_{N}$ (with $\left\lceil\frac{D-1}{2}\right\rceil \geq N \geq 1$ ) with closed orbits with period $2 \pi$ which are defined in a region that covers $\mathscr{H}$ and the entire domain of outer communication. Each of these Killing fields commutes with $t$, and

$$
t=K+\sum_{i=1}^{N} \Omega_{i} \psi_{i}
$$

for some constants $\Omega_{i} \neq 0$, all of whose ratios are irrational. 
The proof of both results relies heavily on the Einstein equations and also on some global results such as topological censorship, see below. First, one shows that near $\mathscr{H}$, the spacetime $\mathscr{M}$ is doubly foliated by a 2-parameter family $B(u, r)$ of compact cross sections, such that the metric takes the "Gaussian null form" [112, 102]

$$
g=2 \mathrm{~d} u\left(\mathrm{~d} r-r \alpha \mathrm{d} u-r \beta_{a} \mathrm{~d} x^{a}\right)+\gamma_{a b} \mathrm{~d} x^{a} \mathrm{~d} x^{b} .
$$

Here, $x^{a}$ are local coordinates on $B$, and $\alpha, \beta=\beta_{a} \mathrm{~d} x^{a}, \gamma=\gamma_{a b} \mathrm{~d} x^{a} \mathrm{~d} x^{b}$ are a scalar field, 1-form, and Riemannian metric on each of the spheres $B(u, r)$ that are parameterized by $u, r$. The horizon $\mathscr{H}$ is at $r=0$. The form of the metric implies that $l=\partial / \partial r$ and $n=\partial / \partial u$ are commuting vector fields. $l$ is a null vector field transverse to $\mathscr{H}$ which is also tangent to a congruence of null geodesics. $n$ is null on $\mathscr{H}$, and on $\mathscr{H}$, tangent to a congruence of null-geodesics, therefore

$$
\nabla_{n} n=\alpha n \quad \text { on } \mathscr{H} .
$$

"Gaussian null coordinates" are adapted particularly well to the geometry near a null surface. The Ricci tensor of $g$ can be expressed in terms of the fields $\alpha, \beta, \gamma$; one obtains (see e.g. [72]):

$$
\begin{aligned}
& R_{a b}=-\mathscr{L}_{l} \mathscr{L}_{n} \gamma_{a b}-\alpha \mathscr{L}_{l} \gamma_{a b}+R_{a b}(\gamma)-\delta^{c}{ }_{(a} \delta^{d}{ }_{b)} D_{c} \beta_{d}-\frac{1}{2} \beta_{a} \beta_{b}+O(r) \\
& R_{u a}=-D_{a} \alpha+\frac{1}{2} \mathscr{L}_{n} \beta_{a}+\frac{1}{4} \beta_{a} \gamma^{b c} \mathscr{L}_{n} \gamma_{b c}-\delta_{[a}^{d} \delta_{b]}{ }_{b]} D_{d}\left(\gamma^{b c} \mathscr{L}_{n} \gamma_{c e}\right)+O(r) \\
& R_{u r}=-2 \mathscr{L}_{l} \alpha+\frac{1}{4} \gamma^{c a} \gamma^{d b}\left(\mathscr{L}_{n} \gamma_{c d}\right) \mathscr{L}_{l} \gamma_{a b}-\frac{1}{2} \gamma^{a b} \mathscr{L}_{l} \mathscr{L}_{n} \gamma_{a b}-\frac{1}{2} \alpha \gamma^{a b} \mathscr{L}_{l} \gamma_{a b}-\frac{1}{2} \beta_{a} \beta^{a}+O(r)
\end{aligned}
$$

Here we have not written out for simplicity terms of order $O(r)$ that vanish on $\mathscr{H}$, but the form of these terms is needed in the proof of the rigidity theorem (see e.g. [72]). The uu, ar and $r r$ components are also not written, but are likewise needed. If $\mathscr{H}$ was already known to be a Killing horizon, then we could choose the foliation $B(u, r)$ in such a way that $n=K$, and hence, in view of eq. (7), that $\alpha=\kappa=$ cst. Even though we do not know this at this stage, the average surface gravity may be defined by

$$
\langle\kappa\rangle=\frac{1}{\operatorname{vol}(B)} \int_{B} \alpha \mathrm{d} S,
$$

where $\mathrm{d} S$ is the volume element associated with $\gamma$, and where $B$ is any of the surfaces $B(u, r=0)$. The average surface gravity turns out to be independent of $u$, because $\alpha$ and in fact any of the tensor fields $r, u, \alpha, \beta, \gamma$ is Lie-derived by $t$.

The desired Killing vector field, $K$, is constructed in two steps.

1. First it is constructed locally in a neighborhood of $H$, in the sense that the 'Taylor expansion' around $\mathscr{H}$ satisfies

$$
\underbrace{\mathscr{L}_{l} \mathscr{L}_{l} \cdots \mathscr{L}_{l}}_{m \text { times }}\left(\mathscr{L}_{K} \Phi\right)=0, \quad m=0,1,2, \ldots \quad \text { on } \mathscr{H},
$$

where $\Phi$ denotes either one of the fields $\alpha, \beta, \gamma$. 
2. Then $K$ is extended to the domain of outer communication. For the purpose of Step 2 ), we assume that the spacetime metric and matter fields be real analytic. The fact that $\mathscr{M}$ is simply connected (see sec. 3.3) implies that the extensions are single valued tensor fields on $\mathscr{M}$.

Thus, if we assume analyticity, the main work of the proof is step 1 ). The key idea of the proof is to choose $K:=n$ for a suitable choice of the foliation $B(u, r)$-to be determined!such that the metric takes Gaussian null form. Note that, since $u, r$ are affine parameters by construction, the foliation is determined uniquely once we give an arbitrary member $B=B(u, r=0)$, and on $B$, we choose $n, l$ subject to $1=g(n, l)$.

To start, one shows that automatically $\mathscr{L}_{n} \gamma=0$, for any foliation of the type described. This is proved via the Raychaudhuri equation

$$
\frac{\mathrm{d}}{\mathrm{d} \lambda} \theta_{n}=-\frac{1}{D-2} \theta_{n}^{2}-\sigma_{n}^{2}-8 \pi T_{u u}
$$

where we mean the expansion $\theta_{n}$ and shear $\sigma_{n}$ of the congruence generated by $n$. The stress energy term $T_{u u}$ actually vanishes due to the vacuum Einstein equations. With the help of this equation, and the global structure of $\mathscr{H}$, one now derives, as in the area theorem, that $\theta_{n}=0=\sigma_{n}$, and because these tensor fields are on $\mathscr{H}$ the trace and trace-free parts of $\mathscr{L}_{n} \gamma$, we are done. However, from the $u a$ component of Einstein's equations written out in Gaussian null-coordinates (3.2) we then get:

$$
\frac{1}{2} \mathscr{L}_{n} \beta=\mathrm{d} \alpha, \quad \text { on } B
$$

and because $\alpha$ is not constant on $B$ unless we choose a very special foliation, we see that $\mathscr{L}_{n} \beta \neq 0$. Thus, for a general foliation, condition (18) already fails for $m=0$, in the case when $\Phi=\beta$. We wish to find a special foliation where this does not happen.

For a given foliation, we may decompose $t=n+s$, where $s$ is tangent to each $B(u, r)$. A key point is that, while the Killing vector field $t$ is given, $n \rightarrow \tilde{n}$ and $s \rightarrow \tilde{s}$ individually change if we change the foliation to $\tilde{B}(\tilde{u}, \tilde{r})$. It turns out that to find the desired foliation $\tilde{B}(\tilde{u}, \tilde{r})$, with corresponding tensor fields $\tilde{u}, \tilde{r}, \tilde{\alpha}, \tilde{\beta}, \tilde{\gamma}$, one has to integrate along the orbit of $s$ two ordinary differential equations on $B$, for the quantities $f=\mathrm{d} \tilde{u} / \mathrm{d} u$ respectively $\tilde{u}$ on $B$. These differential equations are

$$
\mathscr{L}_{s} \log f=\alpha, \quad \mathscr{L}_{s} \tilde{u}=1-\frac{1}{f} .
$$

It furthermore turns out that, once $\tilde{u}$, hence $\tilde{r}, \tilde{\alpha}, \tilde{\beta}, \tilde{\gamma}$, have been determined through (21), the remaining conditions (18) are automatically satisfied for all $m$. The non-obvious proof of this uses Einstein's equations in the form (3.2), but expanded to all orders in $r$. Thus, what remains to be solved is eqs. (21), both of which are schematically of the form

$$
\mathscr{L}_{s} \Psi=J .
$$


When solving this equation with respect to $\Psi$, the spacetime dimension and the topology of $B$ plays a crucial role.

For 4-dimensions, the cross-section $B$ must be topologically a 2-sphere due to Hawking's topology theorem [62]. It then immediately follows that the flow of $s$ on $B$ must have a fixed point, $p$, as the Euler characteristic of $B \cong S^{2}$ is non-zero.

Now the (infinitesimal) action of $s$ on any 2-vector, $v$, on the tangent space at the fixed point $p$ (where $s=0)$, is $\left(\mathscr{L}_{s} v\right)^{a}=-v^{b}\left(D_{b} s^{a}\right)$. Since $D_{b} s^{a}$ is a linear map on the tangent space $T_{p} B$, which is anti-symmetric with respect to $\gamma_{a b}$, the action of $s$ describes an infinitesimal 'rotation' on the tangent space. Therefore all the orbits of $s$ must be closed with a certain period $P$. Then, by integrating Eq. (22) along a closed orbit of $s$ one can always find a well-defined solution $\Psi$ which determines uniquely our desired foliation $\tilde{B}(\tilde{u}, \tilde{r})$. Putting $K=\tilde{n}$, one can furthermore see inductively that eqs. (18) are satisfied for any number of Lie derivatives by taking multiple $\mathscr{L}_{l}$ derivatives of $(3.2)^{7}$. Thus, one has completed step 1). Step 2) is accomplished, as already mentioned, by analytic continuation.

In higher dimensions $D>4$, however, cross-sections $B$ of the event horizon can admit non-trivial topology, and there is no reason that the isometries of $B$ generated by $s$ need to have closed orbits even if $s$ vanishes at some point $p \in \Sigma$. (This would be the case even in 4-dimensions if the horizon topology could be e.g. a torus). An example is supplied by considering a 5-dimensional Myers-Perry black hole solution [107], whose event horizon cross-section is topologically $B \cong S^{3}$. The solution admits two rotational Killing fields, $\psi_{1}, \psi_{2}$ and their linear combination provides $s=\Omega_{1} \psi_{1}+\Omega_{2} \psi_{2}$ on $B$. If we choose two rotational parameters in the linear combination so that their ratio becomes incommensurable-i.e. $\Omega_{1} / \Omega_{2} \notin \mathbb{Q}$-then the orbits of $s$ are not closed on $B$. In this case, the argument which works in $D=4$ simply does not work as stated in higher dimensions.

Let us see in more detail what is the potential issue with eq. (22), and how we can overcome it. First, it turns out that also in higher dimensions, we can also in general always decompose $s$ as $s=\sum_{i} \Omega_{i} \psi_{i}$ for $N$ commuting Killing vector fields $\psi_{i}$ on $(B, \gamma)$ with closed orbits, and corresponding flow (an action of the torus $\mathbb{T}^{N}$ on $B$ ) $F_{\underline{\tau}}$, with $\underline{\tau}=\left(\tau_{1}, \ldots, \tau_{N}\right.$ ) a vector of parameters corresponding to the commuting Killing fields. For each fixed $x \in B$, consider the Fourier transform (with $\underline{m} \in \mathbb{Z}^{N}$ ) in the variables $\tau_{i}$ :

$$
\widehat{J}(x, \underline{m})=\frac{1}{(2 \pi)^{N}} \int_{\mathbb{R}^{N}} J\left[F_{\underline{\tau}}(x)\right] \mathrm{e}^{i \underline{\tau} \cdot \underline{m}} \mathrm{~d}^{N} \tau .
$$

Then a formal solution $\Psi$ to (22) is given by

$$
\Psi(x)=i \sum_{m_{1}, \ldots, m_{N} \in \mathbb{Z} \backslash \underline{0}} \frac{\widehat{J}(x, \underline{m})}{\underline{m} \cdot \underline{\Omega}}=i \sum_{m_{1}, m_{2} \in \mathbb{Z} \backslash \underline{0}} \frac{\widehat{J}\left(x, m_{1}, m_{2}\right)}{m_{1} \Omega_{2}} \cdot\left(\frac{\Omega_{1}}{\Omega_{2}}-\frac{m_{2}}{m_{1}}\right)^{-1} .
$$

\footnotetext{
${ }^{7}$ Here one also has to use the explicit form of the $O(r)$ terms, as well as the remaining Einstein equations.
} 
In the second line, we have restricted to the case $N=2$, to illustrate the potential problem with this expansion. The point is that any irrational number, $\Omega_{1} / \Omega_{2} \notin \mathbb{Q}$, can be approximated by rational numbers $m_{2} / m_{1} \in \mathbb{Q}$ arbitrarily closely, by taking $m_{1}, m_{2}, \rightarrow \infty$ in a suitable manner. This implies that the denominator of the right side of the above equation can become arbitrarily small and therefore that the series for $\Psi$ might not be convergent.

Nevertheless, this difficulty has been overcome for non-extremal black holes (i.e., the case in which $\langle\kappa\rangle \neq 0$ ) by employing a novel approach, and the rigidity result has thereby been generalized to higher dimensions by Refs. [72, 103]. A key new idea employed in Ref. [72] is to apply basic results from ergodic theory (see e.g. [132]) to the flow $F_{\underline{\tau}}$ under consideration. To be able to apply these results, it is important here that $B$ is compact, and that the flow is isometric, i.e. in particular area preserving. When combined with Einstein's equations, the results from ergodic theory enable one to solve the desired differential equations (21) without appealing to the explicit series solution above, and hence avoiding the potential 'small denominator problem'.

Unfortunately, for the extremal black hole case $\langle\kappa\rangle=0$, these methods using ergodic theory do not seem to generalize in a straightforward manner. But if we additionally require (in the example of $N=2$-dimensional torus above), that there exists a $q>0$ such that

$$
\left|\frac{\Omega_{1}}{\Omega_{2}}-\frac{m_{2}}{m_{1}}\right|>\frac{1}{m_{1}^{q}},
$$

then one can show that the formal series solution, $\Psi$, to (22), given by eq. (24), is convergent and therefore well-defined, and that it gives the desired (analytic) solution to our equation. Condition (25) - called Diophantine condition - is known to be satisfied for some $q$ except when $\Omega_{1} / \Omega_{2}$ happens to be in a special set of irrational numbers of measure zero. Therefore, we can virtually always solve eq. (22) [69]. A similar condition can also be formulated for $D \geq 6$, see [69]. Again, this generalized Diophantine condition holds for all $\underline{\Omega} \in \mathbb{R}^{N}$ except for a set of Lebesgue measure zero.

With the additional condition of the type (25), the rigidity theorems above have been extended to include extremal black holes in Theorems 1 and 2 of Ref. [69]. Note that when $N=1$, the Diophantine condition is automatically satisfied. But when $N>1$-which can happen only in higher dimensions - the condition is non-trivial. In this sense, the theorems for the extremal black hole case are weaker than the theorems for the non-extremal case.

A few remarks on the rigidity theorems for both extremal and non-extremal cases are in order:

1. Theorems 1 and 2 above, and those corresponding to the extremal case in Ref. [69] hold also true for stationary black holes coupled to matter fields in a fairly general class of theories that include scalar fields taking values in some Riemannian target space $(X, \mathcal{G})$, Abelian gauge fields with or without a Chern-Simons type term in the action, 
as well as cosmological constant, described by an action of the form

$$
S=\int_{\mathscr{M}} \frac{1}{2} R \star_{D} 1+\frac{1}{2} \mathcal{G}_{a b}(\phi) \mathrm{d} \phi^{a} \wedge \star_{D} \mathrm{~d} \phi^{b}+\mathcal{V}(\phi) \star_{D} 1+\mathcal{H}_{A B}(\phi) F^{A} \wedge \star_{D} F^{B},
$$

plus possibly a Chern-Simons term. The key requirements on $\mathcal{H}, \mathcal{V}$ are essentially that the stress tensor component in the Raychaudhuri equation satisfies the energy condition $T_{u u} \geq 0$, which means that $\mathcal{H}_{A B}$ and $\mathcal{G}_{a b}$ should be positive definite. Thus, the above theorems in particular apply to stationary, asymptotically (anti-)de Sitter black holes as well.

2. The theorems apply not only to a black hole horizon but also to any horizon defined as a "boundary" of the causal past of a complete orbit of some Killing vector field, such as a cosmological horizon if it exists.

3. One can partially remove the analyticity assumption for the black hole interior, following the strategy of Refs. $[44,114]$. For the non-extremal case, the event horizon is isometric to a portion of some bifurcate Killing horizon [116, 117]. Then one can use the bifurcate horizon as an initial data surface for $K$ defined in a neighborhood of $\mathscr{H}$. Then, applying a characteristic initial value formulation to extend $K$ into the interior of the black hole. This type of characteristic initial value problem is ill-defined towards the black hole exterior region and therefore would not appear to be applicable to extend $K$ into the domain of outer communication. Nevertheless, remarkable progress has recently been made along this direction $[82,83]$ in $D=4$. Unfortunately, these methods depend at the moment on the consideration of a tensor field ("Mars Simon tensor" [98]) which has remarkable properties in $D=4$, but which has no obvious generalization to higher dimensions. Thus, it seems that their approach also does not have an obvious generalization to $D \geq 5$.

\subsection{Topology theorems}

We next summarize what is known about the topology of higher dimensional black hole spacetimes, and what the differences to 4 dimensions are. Of interest are:

1. The topology of the event horizon cross sections, $B$.

2. The topology of the domain of outer communications, $\mathscr{M}$.

1) Horizon Topology: The topological (actually $C^{\infty_{-}}$) invariant that is known to characterize the topology of the horizon $B$ is the so-called "Yamabe number". This is defined as follows. Let $\gamma$ be any Riemannian metric on $B$, not necessarily that induced from the 
spacetime metric $g$. Define the number $Y[\gamma, B]$, depending on the conformal class of $\gamma$ by

$$
Y[\gamma, B]:=\inf _{\varphi>0, \varphi \in C^{\infty}} \frac{\int_{B} R(\tilde{\gamma}) \mathrm{d} \tilde{S}}{\left(\int_{B} \mathrm{~d} \tilde{S}\right)^{\frac{D-4}{D-2}}}
$$

where $\tilde{\gamma}=\varphi^{2} \gamma$ is the conformally transformed metric, and $\mathrm{d} \tilde{S}$ its integration element. The Yamabe invariant is given by $Y[B]=\sup _{[\gamma]} Y[B, \gamma]$, where the sup is over all conformal classes. In $D=4$, where $B$ is 2-dimensional, $Y[B]$ is up to a constant equal to the Euler characteristic of $B$.

Einstein's equations give constraints on the Yamabe invariant of horizon cross sections, and in fact also on more general $D-2$-dimensional compact surfaces $B \subset \mathscr{M}$ satisfying certain geometrical conditions. These geometric conditions are that $B$ is a "stably marginally outer trapped surface" (MOTS). This notion is defined as follows, see e.g. [1] for discussion. We suppose that, on $B$, we have defined a pair of null vector fields $n, l$, orthogonal to $B$, such that $g(n, l)=1$. $n$ is chosen future pointing, and is extended to generate a congruence of affine null geodesics, i.e. a null sheet $\mathscr{N} . l$ is chosen "outward pointing", parallel transported along $\mathscr{N}$, and extended off $\mathscr{N}$ by demanding that it be tangent to another congruence of null geodesics. $n, l$ are completely fixed once they have been defined on $B$, and at each point $p$ of $B$, they are uniquely fixed up to a rescaling keeping $g(n, l)=1$. Letting $\theta_{n, l}$ be the respective null expansions, it is demanded that there exists a choice of $n, l$, such that

$$
\theta_{n}=0, \quad \mathscr{L}_{l} \theta_{n} \geq 0, \quad \text { on } B
$$

The first condition states that the expansion on $\mathscr{N}$ in the $n$ direction vanishes, and the second one is satisfied if $\theta_{n}$ is non-negative slightly outside of $\mathscr{N}$. An example of a MOTS is the event horizon $\mathscr{H}$ of a black hole (see [99] (proposition 3) and also [76]), see the following picture, so the following arguments in particular apply to that case. 


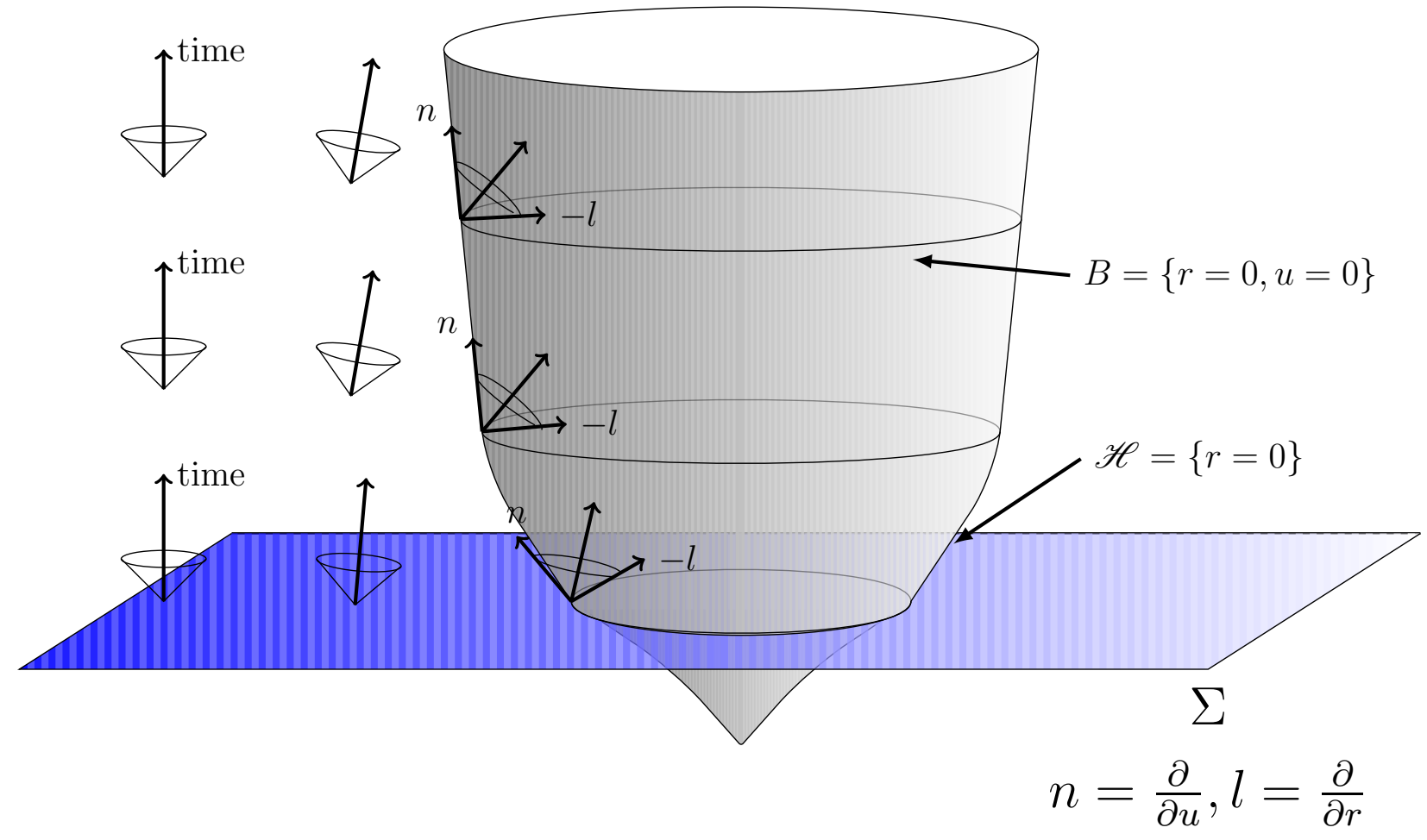

A more pedestrian way of stating the MOTS condition is that, near $\mathscr{N}$, the metric $g$ takes Gaussian null form (14), with $\mathscr{N}$ defined by $r=0, B$ defined by $u=0=r$, and with $n=\partial / \partial u, l=\partial / \partial r$. (Since we take $u$ to be an affine parameter, the metric coefficient of $\alpha$ in (14) is now in fact $r^{2}$ rather than only $r$.) The MOTS conditions are equivalent to $\gamma^{a b} \partial_{u} \gamma_{a b}=0$ and $\partial_{r}\left(\gamma^{a b} \partial_{u} \gamma_{a b}\right) \geq 0$. The theorem is [46, 47], see also [64] for antecedents:

Theorem 3. ("Horizon topology theorem") If $B$ is a stably marginally outer trapped surface, and if the stress tensor satisfies the dominant energy condition, then $B$ is of positive Yamabe type, $Y[B]>0$.

A particularly simple proof of this theorem was given by [115]. To combine the geometric conditions on $B$, and the conditions on the stress tensor, i.e. in effect, the Einstein equations, one first derives from the $u r$ and $a b$ components of the Einstein equations in Gaussian null coordinates (14):

$$
\mathscr{L}_{l} \theta_{n}+\theta_{n} \theta_{l}=T_{u r}+\frac{1}{2}\left[R(\gamma)+D^{a} \beta_{a}-\frac{1}{2} \beta^{a} \beta_{a}\right] .
$$

Then, multiplying both sides by a testfunction $\varphi$, using the MOTS conditions, using $T_{u r} \geq 0$, applying the elementary inequality

$$
\varphi^{2} D^{a} \beta_{a}=D^{a}\left(\varphi^{2} \beta_{a}\right)-2 \varphi\left(D^{a} \varphi\right) \beta_{a} \leq D^{a}\left(\varphi^{2} \beta_{a}\right)+2\left(D^{a} \varphi\right) D_{a} \varphi+\frac{1}{2} \varphi^{2} \beta^{a} \beta_{a}
$$


and integrating over $B$, one gets for $D \geq 5$ :

$$
\frac{\int_{B}\left(4 \frac{D-3}{D-4}\left(D^{a} \varphi\right) D_{a} \varphi+R(\gamma) \varphi^{2}\right) \mathrm{d} S}{\left(\int_{B} \varphi^{\frac{2(D-2)}{D-4}} \mathrm{~d} S\right)^{\frac{D-4}{D-2}}} \geq 0 .
$$

This inequality is known to imply $Y[B, \gamma] \geq 0$, hence $Y[B] \geq 0$ also. For $D=4$, one simply integrates eq. (29) over $B$ and uses Gauss theorem to get rid of the total divergence term. This immediately gives $\int_{B} R(\gamma) \mathrm{d} S \geq 0$, i.e. $B \cong S^{2}$ or $T^{2}$ by the Gauss-Bonnet-theorem. If one assumes the strict MOTS condition $\left(\mathscr{L}_{l} \theta_{n}>0\right.$ somewhere on $\left.B\right)$, one obtains the claim $Y[B]>0$. Actually, one can even show that $Y[B]>0$ without the strict MOTS condition, by another argument $[46,47]$.

The condition that $Y[B]>0$ imposes a restriction on the possible topologies of $B$. For example:

1. $D=4: Y[B]>0$ implies that the Euler characteristic of $B$ is positive, hence $B \cong S^{2}$. Thus, one recovers Hawking's topology theorem [62].

2. $D=5$ : In this case $B$ is a closed compact 3 -manifold. It is known that $Y[B]>0$ implies that $B$ is a connected sum

$$
B \cong \#_{i}\left(S^{3} / \Gamma_{i}\right) \quad \# k \cdot\left(S^{2} \times S^{1}\right)
$$

where $\Gamma_{i} \subset O(4)$ are discrete subgroups (possibly trivial).

3. In dimensions $D>5$, the positive Yamabe condition becomes less and less restrictive.

Further restrictions arise e.g. in $D=5$ in the case of stationary black holes, if we combine these techniques with the topological censorship theorem (see below) and the rigidity theorem (see above). In that case, the rigidity theorem guarantees that the spacetime metric has a $U(1)$ symmetry in addition to being stationary. In particular, $B$ has an action of $U(1) \cong S^{1}$, and so is a special case of a Seiffert 3 -manifold. One can consider the factor space $\hat{B}=B / U(1)$, which in general is not a manifold, but an "orbifold" (possibly with boundaries), characterized by singular points with deficit angles $2 \pi / p_{i}$, where $p_{i} \in \mathbb{Z}_{+}$. Then, using Einstein's equation, one derives that the genus of $\hat{B}$ satisfies

$$
\chi_{\text {orbifold }}(\hat{B})=2-2 \operatorname{genus}(\hat{B})-\sum_{i}\left(1-\frac{1}{p_{i}}\right)>0,
$$

if $\hat{B}$ is closed, and one also derives constraints from the topological censorship theorem if $\hat{B}$ has a boundary. The quantity on the left side is known as an invariant called "orbifold Euler characteristic" of the Seiffert fibration $B \rightarrow \hat{B}$. For the case of positive orbifold Eulercharacteristic, a complete classification of such fibrations is available in the mathematics literature, and this leads to [68]: 
Theorem 4. ("Refined topology theorem") For a stationary black hole in $D=5$ in a theory for which the topological censorship theorem and rigidity theorem hold, we must have

$$
B \cong\left\{\begin{array}{l}
S^{3} / \Gamma \\
\# k \cdot\left(S^{2} \times S^{1}\right) \quad \#_{i} L\left(p_{i}, q_{i}\right)
\end{array}\right.
$$

where the list of possible subgroups $\Gamma \subset O(4)$ is given in [68], and where each $L(p, q)$ is a lens space.

The lens space corresponds to a particular quotient, but $\Gamma$ in the first line can be more general, and $S^{3} / \Gamma$ includes also e.g. prism spaces, Poincare homology spheres etc.

Yet further restrictions arise in $D=5$ and higher dimensions if one assumes that the spacetime carries an isometric action not just of a single copy of $U(1)$, generic in view of the rigidity theorem, but instead of multiple copies of $U(1)$, most likely non-generic. The most stringent restrictions are obtained if the isometry group contains $U(1)^{D-3}$. Then it can be shown that each horizon component must be one of the following [74]:

$$
B \cong\left\{\begin{array}{l}
S^{3} \times \mathbb{T}^{D-5} \\
S^{2} \times \mathbb{T}^{D-4} \\
L(p, q) \times \mathbb{T}^{D-5}
\end{array}\right.
$$

In particular, in $D=5$ we only have the possibilities $S^{3}, S^{2} \times S^{1}, L(p, q)$. In particular, more exotic quotients of $S^{3}$ other than the lens space are ruled out if the symmetry group contains $U(1)^{2}$.

b) Domain of outer communication: Much less is known about the possible topology of the domain of outer communication in general, except for static black holes (see sec. 3.8), where the topology of the Cauchy surface $\Sigma$ connecting the horizon(s) and spatial infinity is flat space minus one ball per black hole horizon, $\mathbb{R}^{D-1} \backslash \cup_{k} B_{k}^{D-1}$. For stationary, but non-static, no comparable result is known, even though this seems to be the situation for most known black holes. For example, for Kerr the Cauchy surface $\Sigma$ also has topology $\Sigma \cong \mathbb{R}^{3} \backslash B^{3}$. The analogous statement is true for Myers-Perry black holes. Similarly, for the black ring in 5 dimensions, $\Sigma \cong \mathbb{R}^{4} \backslash\left(S^{1} \times B^{2}\right)$.

A simple general statement, which already follows from the requirement of global hyperbolicity without even using Einstein's equations, is that $\mathscr{M} \cong \mathbb{R} \times \Sigma$. Another general statement, which uses Einstein's equations, is the "topological censorship theorem" [43, 45, 22], already alluded to above. In the most general form, it can be stated as:

Theorem 5. ("Topological censorship theorem") Let Einstein's equation hold with a stress tensor satisfying the null convergence condition $T_{k k} \geq 0$ for any null vector $k$. If the spacetime is asymptotically flat or asymptotically Kaluza-Klein, then any curve $c$ in $\mathscr{M}$ (or in $\Sigma$ ) with beginning and endpoint in the asymptotic region can be continuously deformed to a curve $\tilde{c}$ that is entirely within the asymptotic region. 
In $D=4$, this theorem implies that $\Sigma$ is simply connected, and in particular furnishes an independent proof that the horizon topology $B \cong S^{2}$ : Indeed, suppose $B$ had handles. Consider a curve with endpoints at infinity that is threaded through one of the handles of $B$. Such a curve clearly could not be smoothly deformed to a curve that is entirely in the asymptotic region, a contradiction with the topological censorship theorem.

In any dimension $D \geq 5$, the topological censorship theorem implies that $\mathscr{M} \cong \mathbb{R} \times \Sigma$ (hence $\Sigma$ ) is simply connected in the asymptotically flat case. This fact was already used in the proof of the rigidity theorem, but it does not restrict the topology of $\Sigma$ very much in $D \geq 5$ dimensions. Tighter restrictions arise if we also use the $U(1)$ symmetry of the spacetime, which is guaranteed by the rigidity theorem.

1. As before, the restriction is most stringent in $D=5$, in which case $\Sigma$ is a simply connected, asymptotically Euclidean, 4-manifold with inner boundary $B$. Then, using results from topology $[110,111,41]$, it was shown in [68] that

Theorem 6. For a stationary asymptotically flat spacetime in a theory in which the assumptions of the rigidity and topological censorship theorem are satisfied, we have

$$
\Sigma \cong\left[\# m \cdot\left(S^{2} \times S^{2}\right) \quad \# m^{\prime} \cdot \mathbb{C} P^{2} \# m^{\prime \prime} \cdot \overline{\mathbb{C} P^{2}} \# \mathbb{R}^{4}\right] \backslash \mathrm{BH}
$$

where $B H$ is a compact 4 -manifold with boundary $B$.

If the spacetime is spin, then the complex projective spaces are absent in the decomposition. In all known solutions, the handles $S^{2} \times S^{2}$ are also absent. This result should maybe be contrasted with the decomposition of general 4-manifolds. As is wellknown, with any compact oriented 4-manifold $X$ one can associate a canonical pairing $Q_{X}: H_{2}(X, \mathbb{Z}) \times H_{2}(X, \mathbb{Z}) \rightarrow \mathbb{R}$. That pairing associates with a pair of 2-cycles $C, C^{\prime}$ the intersection number $\#\left(C \cap C^{\prime}\right)$, where each intersection point is counted with a \pm sign determined by the relative orientations. $Q_{X}$ defines a quadratic form with entries in $\mathbb{Z}$. A deep theorem in topology [42] states that $Q_{X}$ can be decomposed up to a similarity transformation as

$$
Q_{X}=m \cdot\left(\begin{array}{ll}
0 & 1 \\
1 & 0
\end{array}\right) \oplus I_{m^{\prime}} \oplus\left(-I_{m^{\prime \prime}}\right) \oplus m^{\prime \prime \prime} \cdot E_{8}
$$

for some $m, \ldots, m^{\prime \prime \prime} \in \mathbb{Z}_{+}$, where $E_{8}$ is the Cartan matrix of the exceptional Lie algebra with the same name, and $I_{m}$ the $m$-dimensional identity matrix. Furthermore, $X$ is determined topologically by its $Q_{X}$ uniquely. If the handle decomposition of $X$ only has $S^{2} \times S^{2}$ 's (corresponding to $m$ ) and complex projective spaces $\mathbb{C} P^{2}$ (corresponding to $m^{\prime}$ ) and $\overline{\mathbb{C} P^{2}}$ (corresponding to $m^{\prime \prime}$ ), then one can see $[110,111]$ that $m^{\prime \prime \prime}=0$, and the $E_{8}$-factors are absent. (If the manifold is spin, the the complex projective spaces are also absent, $m^{\prime}=0=m^{\prime \prime}$.) This result cannot be applied directly to $\Sigma$, because it 
is not a compact manifold. However, we can glue in a ball at infinity, and a suitable manifold at the horizon (see [74] for details) to obtain from $\Sigma$ a compact 4-manifold in a canonical way, which admits again an action of $U(1)^{2}$. If we call by abuse of notation the quadratic form of this manifold by $Q_{\Sigma}$, then we have:

Theorem 7. For a rotating stationary black hole $\mathscr{M} \cong \mathbb{R} \times \Sigma$, in $D=5$, the decomposition of $Q_{\Sigma}$ does not contain $E_{8}$ 's.

2. In higher dimensions $D \geq 6$, the topological censorship theorem combined with the $U(1)$ symmetry from the rigidity theorem, does not seem to give a lot of restriction. However, assume that we even have an action of $U(1)^{D-3}$. We conjecture:

Conjecture 1. Assume that $(\mathscr{M}, g)$ is spin, asymptotically flat or KK, has a Riccitensor satisfying the null-convergence condition, and admits an action of $U(1)^{D-3}$. Then the Cauchy surface $\Sigma$ can be decomposed as

$$
\Sigma \cong\left[\#_{k=2}^{D-3} m_{k} \cdot\left(S^{k} \times S^{D-1-k}\right) \quad \#(\text { asymptotic region })\right] \backslash \mathrm{BH},
$$

where the asymptotic region depends on the precise boundary conditions; e.g. in the standard KK setup $\mathbb{R}^{3} \times \mathbb{T}^{D-4}$.

\subsection{Weyl-Papapetrou coordinates and orbit space analysis}

An important step in the uniqueness proof of the Kerr-Newman metric in $D=4$ is to bring the metric into the Weyl-Papapetrou form. In 4 dimensions, the Weyl-Papapetrou form follows from the existence of the commuting Killing fields $t, \psi$ and certain regularity assumptions about the global causal structure alone, whereas the existence of $\psi$ in turn follows form the rigidity theorem, and $t$ is assumed to start with. Thus, the Weyl-Papapetrou form of the metric for stationary black essentially (i.e., modulo the technical assumptions described in sec. 3.1) does not imply any loss of generality for stationary metrics. However, in $D \geq 5$, the Weyl-Papapetrou form only seems to follow if one makes the assumption that the number $N$ of rotational Killing fields is $N=D-3$. The rigidity theorem only guarantees $N \geq 1$ rotational Killing fields, and this might well be the generic case. Thus, it is likely that the Weyl-Papapetrou form is not generic in higher dimensions, even in vacuum Einsteingravity. Nevertheless, if one does assume the existence of $D-3$ commuting axial Killing fields, (i.e. an isometric action of $U(1)^{D-3}$ on $(\mathscr{M}, g)$ ), then the generalized Weyl-Papapetrou can be shown to follow:

Theorem 8. ("Weyl-Papapetrou-form") If the spacetime is asymptotically Kaluza-Klein with 3, 4, 5 asymptotically flat ("large") dimensions, satisfies the vacuum Einstein equations, if there are commuting Killing fields $t, \psi_{1}, \ldots, \psi_{D-3}$ generating the isometry group $G=$ 
$\mathbb{R} \times U(1)^{D-3}$, and if the spacetime satisfies satisfies a causal regularity condition mentioned in sec. 3.1, then the metric can be brought into Weyl-Papapetrou form

$$
g=-\frac{r^{2} \mathrm{~d} \tau^{2}}{\operatorname{det} f}+\mathrm{e}^{-\nu}\left(\mathrm{d} r^{2}+\mathrm{d} z^{2}\right)+f_{i j}\left(\mathrm{~d} \varphi^{i}+w^{i} \mathrm{~d} \tau\right)\left(\mathrm{d} \varphi^{j}+w^{j} \mathrm{~d} \tau\right)
$$

globally, but away from the horizon $\mathscr{H}$ and any axis of rotation.

The notation for the coordinates in the Weyl-Papapetrou form is analogous to that given before in 4 dimensions: $\varphi^{i}$ are $2 \pi$-periodic coordinates such that the rotational Killing fields $\psi_{1}, \ldots, \psi_{D-3}$ take the form $\psi_{i}=\partial / \partial \varphi^{i}$, and $\tau$ is a coordinate such that the timelike Killing field takes the form $t=\partial / \partial \tau$. In other words, the metric functions $f_{i j}, w^{i}, \nu$ are independent of $\tau, \varphi^{1}, \ldots, \varphi^{D-3}$, and only depend on $z \in \mathbb{R}, r>0$. The argument establishing (39) is outlined below.

It is important to emphasize that the Weyl-Papapetrou form does not reflect in a very transparent way either the global nature of $\mathscr{M}$, nor the global nature of the action of the symmetry group on $\mathscr{M}$ in $D \geq 5$. Both have to be taken into account and understood to appreciate the meaning of the coordinates, and to actually derive the form of the metric. Broadly speaking, the points labeled by $r=0$ describe points in $\mathscr{M}$ which are either (i) on the horizon $\mathscr{H}$, or (ii) lie on an "axis of rotation". By the latter one means in higher dimensions points such that a non-trivial linear combination of the rotational Killing fields $\psi_{i}$ vanishes. In case (i), this follows from the fact that $t=\partial / \partial \tau$ has zero norm on $\mathscr{H}$. For (ii), this can be seen from the fact that, for such points $f_{i j}$ fails to have full rank, hence $\operatorname{det} f=0$, hence from the first term in $g, r=0$. This will be described in more detail when we come to the proof of (39). In particular, we will describe how non-trivial topologies of $\mathscr{M}$ are compatible with (39). We also note that the relation between $r, z$, and the asymptotically Cartesian (spatial) coordinates $x_{1}, \ldots, x_{s}$ in the asymptotically KK-region (see sec. 3.1) is

$$
(r, z) \sim \begin{cases}\left(\sqrt{x_{1}^{2}+x_{2}^{2}}, x_{3}\right) & \text { if } s=3 \\ \left(\sqrt{\left(x_{1}^{2}+x_{2}^{2}\right)\left(x_{3}^{2}+x_{4}^{2}\right)}, \frac{1}{2}\left(x_{1}^{2}+x_{2}^{2}-x_{3}^{2}-x_{4}^{2}\right)\right) & \text { if } s=4\end{cases}
$$

The theorem 8 can be proved also for more general Einstein-matter systems, see sec. 4 . The proof of this result is rather more non-trivial than in $D=4$, due to new global considerations about the topology of $\mathscr{M}$ and the nature of the action of the isometry group $G=\mathbb{R} \times U(1)^{D-3}$. Therefore we will outline it here, for details see [74]. For definiteness, we will now outline the proof in the case of vacuum general relativity.

Because the symmetry group has $(D-2)$ dimensions, the metric will, in a sense, depend non-trivially only on two remaining coordinates, and the Einstein equations will consequently reduce to a coupled system of PDE's in these variables $(r, z$ above, but we need to explain how exactly we choose them!). However, before one can study these equations, one must understand more precisely the nature of the two remaining coordinates, or, mathematically speaking, the nature of the orbit space $\mathscr{M} /\left[\mathbb{R} \times U(1)^{D-3}\right]$. The quotient by $\mathbb{R}$ simply gets rid 
of a global time coordinate, so one is left with the quotient of a spatial slice $\Sigma$ by $U(1)^{D-3}$. To get an idea about the topological properties of this quotient, we consider the following two simple, characteristic examples in the case $\operatorname{dim} \Sigma=4$, i.e. $D=5$.

The first example is $\Sigma=\mathbb{R}^{4}$, with one factor of $U(1) \times U(1)$ acting by rotations in the 12 -plane and the other in the 34-plane. Introducing polar coordinates $\left(R_{1}, \varphi_{1}\right)$ and $\left(R_{2}, \varphi_{2}\right)$ in each of these planes, the group shifts $\varphi_{1}$ resp. $\varphi_{2}$, and the quotient is thus given simply by the first quadrant $R_{1}>0, R_{2}>0$, which is a 2-manifold with two boundary components, i.e. the semi-axis, and the corner where the two axis meet. The first boundary component corresponds to places in $\mathbb{R}^{4}$ where the Killing field $\psi_{1}=\partial / \partial \varphi_{1}$ vanishes, whereas the second boundary component to places where $\psi_{2}=\partial / \partial \varphi_{2}$ vanishes. On the corner, both Killing fields vanish and the group action has a fixed point. The second example is the cartesian product of a plane with a 2-torus, $\Sigma=\mathbb{R}^{2} \times \mathbb{T}^{2}$. Letting $\left(x_{1}, x_{2}\right)$ be cartesian coordinates on the plane, and $\left(\varphi_{1}, \varphi_{2}\right)$ angles on the torus, suppose the group action is generated by the vector fields $\psi_{1}=\partial / \partial \varphi_{1}$ and by $\psi_{2}=\alpha \partial / \partial \varphi_{2}+\beta\left(x_{1} \partial / \partial x_{2}-x_{2} \partial / \partial x_{1}\right)$, where $\alpha, \beta$ are integers. These vector fields do not vanish anywhere, but there are discrete group elements leaving certain points invariant. The quotient is now a cone with deficit angle $2 \pi / \alpha$.

The general case turns out to be locally the same as in these examples $[110,111](D=5)$ [73] (general $D$ ). In fact, one can show that the quotient $\Sigma /[U(1) \times U(1)]$ is a 2-dimensional conifold with boundaries and corners. Each boundary segment is characterized by a different pair $(p, q)$ of integers such that $p \psi_{1}+q \psi_{2}=0$ at corresponding points of $\Sigma$, see fig. 1 .

For subsequent boundary segments adjacent on a corner labeled by $\underline{v}_{i}=\left(p_{i}, q_{i}\right)$ and $\underline{v}_{i+1}=\left(p_{i+1}, q_{i+1}\right)$, we have the condition

$$
\operatorname{det}\left(\begin{array}{cc}
p_{i} & p_{i+1} \\
q_{i} & q_{i+1}
\end{array}\right)= \pm 1
$$

Each conical singularity is characterized by a deficit angle, i.e. another integer. In higher dimensions, there is a similar result; now a boundary segment is e.g. characterized by $(D-3)$ tuple of integers $\underline{v}_{i}=\left(v_{1 i}, \ldots, v_{(D-3) i}\right)$ ("winding numbers"), and the compatibility condition at the corners is somewhat more complicated, see the theorem below.

In the case where $\Sigma$ is the spatial section of a black hole spacetime, there are further constraints coming from Einstein's equations, and the orientability of the spacetime. The topological censorship theorem is seen to imply, using various methods in algebraic topology, that the 2-dimensional orbit space $\hat{\Sigma}=\Sigma / U(1)^{D-3}$ cannot have any conifold points, nor holes, nor handles, and therefore has to be diffeomorphic to an upper half plane $\hat{\Sigma} \cong\{(r, z) \mid$ $r>0\}$. The boundary segments correspond to intervals on the boundary $(r=0)$ of this upper half plane and are places ("axis") in the manifold $M$ where a linear combination of the rotational Killing fields vanish, or to a horizon. Here the Killing fields do not vanish except where an "axis" meets a horizon. Furthermore, with each boundary segment $\left(z_{J}, z_{J+1}\right)$, one can associate its length $l_{J} \geq 0$ via the metric $g$ (see below). 


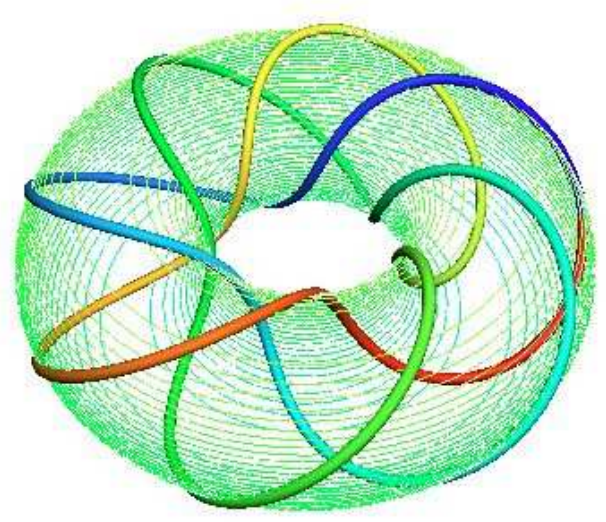

Figure 1: The numbers $(p, q)$ may be viewed as winding numbers associated with the generators of the 2-torus generated by the two axial Killing fields. In such a torus, an $U(1)$-orbit winds around the first $S^{1}$ generator $n$-times as it goes $p$-times around the other $S^{1}$-direction. Here $(q n \equiv 1 \bmod p)$. The figure shows the situation for $p=3, n=7$.

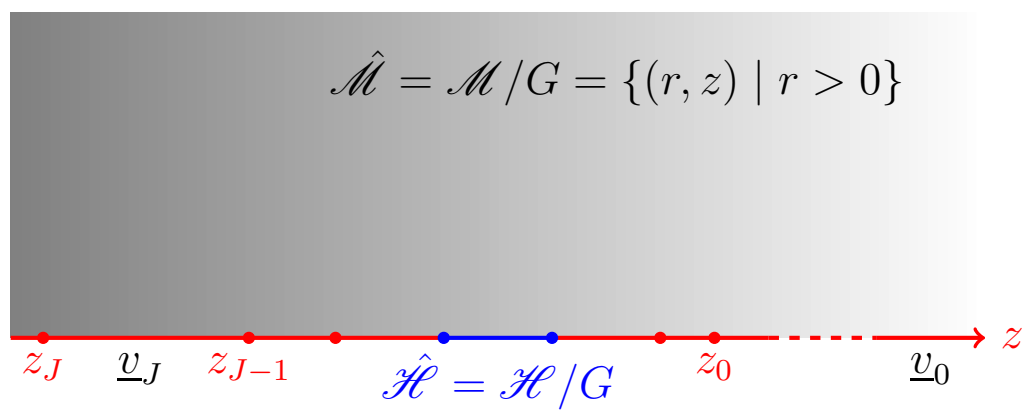

Thus, in summary, one has [74]:

Theorem 9. ("Orbit space theorem") Let $(\mathscr{M}, g)$ be asymptotically flat or KK, with Ricci tensor satisfying the null-convergence condition. If one assumes the isometry group $G=$ $\mathbb{R} \times U(1)^{D-3}$, then the orbit space $\hat{\mathscr{M}}=\mathscr{M} / G$ is homeomorphic to an upper half plane $\{(r, z) \mid r>0\}$. Furthermore, the boundary $r=0$ can be thought of as divided up into 
a collection of intervals $\left(-\infty, z_{1}\right),\left(z_{1}, z_{2}\right), \ldots,\left(z_{n},+\infty\right)$, each of which either represents the orbit space $\hat{\mathscr{H}}=\mathscr{H} / G$ of the horizon (one interval per horizon component, if multiple horizons are present), or an axis in the spacetime where a linear combination $\sum_{i} v_{i J} \psi_{i}$ of the rotational Killing fields vanishes. The quantity $\underline{v}_{J}=\left(v_{1 J}, \ldots, v_{(D-3) J}\right) \in \mathbb{Z}^{D-3}$ is a vector associated with the $J$-th interval which necessarily has integer entries ${ }^{8}$. For adjacent intervals $J$ and $J+1$ (not including the horizon), there is a compatibility condition stating that the collection of minors $Q_{k l} \in \mathbb{Z}, 1 \leq k<l \leq D-3$ given by

$$
Q_{k l}=\left|\operatorname{det}\left(\begin{array}{cc}
v_{k(J+1)} & v_{k J} \\
v_{l(J+1)} & v_{l J}
\end{array}\right)\right|
$$

have greatest common divisor g.c.d. $\left\{Q_{k l}\right\}=1$

Some examples in $D=5$ are summarized in the following tables

\begin{tabular}{|c|c|c|c|}
\hline & Interval Lengths & Vectors (Labels) & Horizon \\
\hline Myers-Perry & $\infty, l_{1}, \infty$ & $(1,0),(0,0),(0,1)$ & $S^{3}$ \\
\hline Black Ring & $\infty, l_{1}, l_{2}, \infty$ & $(1,0),(0,0),(1,0),(0,1)$ & $S^{2} \times S^{1}$ \\
\hline Black Saturn & $\infty, l_{1}, l_{2}, l_{3}, \infty$ & $(1,0),(0,0),(0,1),(0,0),(0,1)$ & $S^{3}$ and $S^{2} \times S^{1}$ \\
\hline Black String & $\infty, l_{1}, \infty$ & $(1,0),(0,0),(1,0)$ & $S^{1} \times S^{2}$ \\
\hline Black Di-Ring & $\infty, l_{1}, l_{2}, l_{3}, l_{4}, \infty$ & $(1,0),(0,0),(1,0),(0,0),(1,0),(0,1)$ & $2 \cdot\left(S^{1} \times S^{2}\right)$ \\
\hline Orth. Di-Ring & $\infty, l_{1}, l_{2}, l_{3}, l_{4}, \infty$ & $(1,0),(0,0),(1,0),(0,1),(0,0),(0,1)$ & $2 \cdot\left(S^{1} \times S^{2}\right)$ \\
\hline Minkowski & $\infty, \infty$ & $(1,0),(0,1)$ & - \\
\hline
\end{tabular}

The explicit form of the metric may be found in [107] (Myers-Perry), [113, 32] (Black ring), [35] (Saturn), [79] (Di-Ring), and [81] (Orthogonal Di-Ring). The following interval structure would represent a "Black Lens", respectively an exotic spherical black hole embedded in an ambient space containing a factor of $\mathbb{C} P^{2}$, if such solutions would exist:

\begin{tabular}{|l|c|c|c|}
\hline & Interval Lengths & Vectors (Labels) & Horizon Topology \\
\hline Black Lens & $\infty, l_{1}, l_{2}, \infty$ & $(1,0),(0,0),(1, p),(0,1)$ & $L(p, 1)$ \\
\hline Exotic MP & $\infty, l_{1}, l_{2}, l_{3}, l_{4}, \infty$ & $(1,0),(0,0),(0,1),(1,1),(1,0),(0,1)$ & $S^{3}$ \\
\hline
\end{tabular}

In these tables, the interval $(0,0)$ corresponds to a horizon. If $\underline{v}_{h-1}$ resp. $\underline{v}_{h+1}$ represent the vectors adjacent to a horizon interval $\left(z_{h}, z_{h+1}\right)$ then the parameter $p=\left|\operatorname{det}\left(\underline{v}_{h-1}, \underline{v}_{h+1}\right)\right|$ is related to the different horizon topologies by:

Note also that the first and last vector $\underline{v}_{0}, \underline{v}_{N}$ in the above solutions is always $(1,0)$ resp. $(0,1)$. This corresponds to the fact that these 5-dimensional solutions are asymptotically flat in all 5 directions. For 5 -dimensional solutions which are asymptotically Kaluza-Klein with one compactified extra dimension, the first and last vectors would be equal, e.g. $(0,1)$ and $(0,1)$. In general, the relationship between $\underline{v}_{0}, \underline{v}_{N}$ and the asymptotic conditions is as follows. First we form the parameter $p=\left|\operatorname{det}\left(\underline{v}_{0}, \underline{v}_{N}\right)\right|$. Then

\footnotetext{
${ }^{8}$ It is unique up to sign if we impose, as we will, that g.c.d. $\left(v_{1 J}, \ldots, v_{(D-3) J}\right)=1$.
} 


\begin{tabular}{c|c}
$p$ & Topology of $\mathscr{H}$ \\
\hline 0 & $S^{2} \times S^{1}$ \\
\pm 1 & $S^{3}$ \\
other & $L(p, q)$
\end{tabular}

Figure 2: The invariant $p=\left|\operatorname{det}\left(\underline{v}_{h-1}, \underline{v}_{h+1}\right)\right|$ characterizes the different horizon topologies.

\begin{tabular}{c|c}
$p$ & Asymptotic conditions \\
\hline 0 & $\mathbb{R}^{3,1} \times \mathbb{T}($ Kaluza-Klein $)$ \\
\pm 1 & $\mathbb{R}^{4,1}$ (Minkowskian) \\
other & $\mathbb{R}^{4,1} / \mathbb{Z}_{p}$ (locally Minkowskian)
\end{tabular}

Figure 3: The invariant $p=\left|\operatorname{det}\left(\underline{v}_{0}, \underline{v}_{N}\right)\right|$ characterizes the different possible asymptotic behaviors.

The last item in the table means that the 4-dimensional spatial slice in the asymptotic region is locally asymptotically Euclidean, i.e. a large sphere $S^{3}$ near spatial infinity is replaced by the quotient $S^{3} / \mathbb{Z}_{p}$ of $S^{3}$ by a discrete cyclic group. Trivial examples of such spacetimes are $g=-\mathrm{d} \tau^{2}+\mathrm{d} s_{\text {Instanton, }}^{2}$ where the spatial part is a suitable 4-dimensional gravitational instanton. These static spacetimes of course do not contain a black hole, but black hole spacetimes of this nature have been constructed by [18].

The numbers $\left\{l_{J}\right\}$ and the assignment of the labels $\left\{\underline{v}_{J}\right\}$ were also considered from a local perspective by [61], [59]. However, we note that, in these references, neither the condition that the components be integers, nor the determinant conditions for adjacent interval vectors and their relation to the horizon topology were obtained. Furthermore, the interval vectors considered in [59] have $D-2$ components, rather than $D-3$.

So far our considerations have been essentially topological; in particular we have not said how exactly the coordinates $(r, z)$ on $\hat{\mathscr{M}}=\mathscr{M} / G$ are to be chosen relative to the metric $g$. The key trick, generalized from [17], is now to make a special choice using the Einstein equations. First, $r$ is defined by

$$
r^{2}=-\operatorname{det}\left(\begin{array}{cc}
g(t, t) & g\left(t, \psi_{i}\right) \\
g\left(\psi_{i}, t\right) & g\left(\psi_{i}, \psi_{j}\right)
\end{array}\right)
$$

That the right side is positive, or equivalently, that the span of $t, \psi_{1}, \ldots, \psi_{D-3}$ is a timelike subspace in each tangent space $T_{x} \mathscr{M}$, is actually rather non-obvious, and global arguments are required to demonstrate it [20]. However, it then immediately follows that $\hat{\mathscr{M}}$ inherits a Riemannian metric, $\mathrm{d} \hat{s}_{2}^{2}$, and it furthermore follows from the vacuum Einstein equations that $r$ is a harmonic function on $\hat{\mathscr{M}}$ w.r.t. this Riemannian metric. It can be seen from this fact, together with an application of the maximum principle and the uniformization theorem [20], that $r$ is a well-defined coordinate on $\hat{M}$, i.e. that its gradient cannot vanish. 
We may supplement $r$ by a second, globally defined, conjugate harmonic coordinate $z$ on $\hat{\mathscr{M}}$

$$
\mathrm{d} r=\star_{2} \mathrm{~d} z .
$$

Together, $(r, z)$ then provide the desired geometrically preferred coordinate system on $\hat{\mathscr{M}}=$ $\{(r, z) \mid r>0\}$. The interval lengths $l_{J}$ are defined by $l_{J}=z_{J+1}-z_{J}$ in this special coordinate system. The induced metric on $\hat{\mathscr{M}}$ can be written by construction as $\mathrm{d} \hat{s}_{2}^{2}=\mathrm{e}^{-\nu(r, z)}\left(\mathrm{d} z^{2}+\mathrm{d} r^{2}\right)$ for some function $\nu$, because $(r, z)$ is a conjugate pair.

The $(r, z)$ coordinates can then complemented by coordinates $\tau \in \mathbb{R}$, and $2 \pi$-periodic coordinates $\varphi^{1}, \ldots, \varphi^{D-3}$ in such a way that the Killing fields are $t=\partial / \partial \tau, \psi_{i}=\partial / \partial \varphi^{i}$. The final step is to show that the family of subspaces $\operatorname{span}\left(t, \psi_{1}, \ldots, \psi_{D-3}\right)^{\perp} \subset T \mathscr{M}$ is integrable. This is shown via Frobenius' theorem, in combination with Einstein's equation. In other words, we see in this way that the metric does not have any cross terms between $\left(\tau, \varphi^{1}, \ldots, \varphi^{D-3}\right)$, respectively $(r, z)$, and the Weyl-Papapetrou form of the metric (39) then follows.

In view of generalizations to more general systems of equations, it is maybe useful to show exactly how integrability is proven. By the "differential form version" of Frobenius' theorem, we need to show that $\mathrm{d} \xi_{I}=\sum_{J} \alpha_{I J} \wedge \xi_{J}$, where $\xi_{I}$ collectively denotes the $D-2$ Killing fields, identified with 1 -forms using the metric. This is equivalent to

$$
\xi_{1} \wedge \cdots \wedge \xi_{D-2} \wedge \mathrm{d} \xi_{J}=0 \text { for all } J
$$

Taking a Hodge-dual of this equation and applying d, gives, using Einstein equations, standard identities for Killing vectors, and the commuting nature of the Killing fields,

$$
\mathrm{d} \star_{D}\left(\xi_{1} \wedge \cdots \wedge \xi_{D-2} \wedge \mathrm{d} \xi_{J}\right)=-8 \pi \star_{D}\left[\xi_{1} \wedge \cdots \wedge \xi_{D-2} \wedge T\left(\xi_{J}\right)\right]
$$

where $T\left(\xi_{J}\right)$ is the 1-form obtained by dotting the vector $\xi_{J}$ into one of the indices of the stress tensor. The right hand side of course vanishes automatically in the vacuum, but also e.g. for the class of theories described by (26) (assuming all the matter fields are Lie-derived by the $\left.\xi_{I}\right)$. Thus, we see that $\star_{D}\left(\xi_{1} \wedge \cdots \wedge \xi_{D-2} \wedge d \xi_{J}\right)$ is a constant. But we also know from the above analysis of the orbit space that at least one linear combination of the $\xi_{I}$ must vanish somewhere on $\mathscr{M}$, hence it is zero, so we are done.

\subsection{Non-linear sigma-model reduction}

The Weyl-Papapetrou form considerably constrains the metric, but it is still not well-suited either to the proof of uniqueness, or the generation of new solutions from old ones. For this, yet another set of ideas is required, namely the embedding of dimensionally reduced gravity models into certain non-linear sigma-models. (The oldest manifestation of this fundamental idea is the discovery by Ehlers [30] of a "hidden" $S L(2)$ symmetry of the stationary axisymmetric Einstein equations.) This embedding is best described if we forget, again, for 
the moment about the asymptotically time-like Killing vector. Then the metric (39) can be written in standard Kaluza-Klein form,

$$
g=(\operatorname{det} f)^{-1} g_{3}+f_{i j}\left(\mathrm{~d} \varphi^{i}+A^{i}\right)\left(\mathrm{d} \varphi^{j}+A^{j}\right)
$$

where $g_{3}$ is the induced line-element on the orbit space and time, $\hat{\mathscr{M}} \times \mathbb{R}$ (parameterized by the coordinates $(\tau, r, z)$ ), and where each $A^{i}$ is a one-form on this space. Explicitly,

$$
g_{3}=-r^{2} \mathrm{~d} \tau^{2}+\operatorname{det} f \mathrm{e}^{-\nu}\left(\mathrm{d} r^{2}+\mathrm{d} z^{2}\right), \quad A^{i}=w^{i} \mathrm{~d} \tau .
$$

One now takes this metric and plugs it into the field equations; for simplicity we will focus on the vacuum case, but other cases will be discussed below in sec. 4. This gives the "Maxwell equation"

$$
\mathrm{d}\left(\operatorname{det} f \cdot f_{i j} \star_{3} \mathrm{~d} A^{j}\right)=0
$$

implying locally the existence of potentials $\chi_{i}$ on $\mathbb{R} \times \hat{\mathscr{M}}$ satisfying

$$
\mathrm{d} \chi_{i}=2 \operatorname{det} f \cdot f_{i j} \star_{3} \mathrm{~d} A^{j} .
$$

These so-called "twist potentials" are in fact defined globally [74]. Following Maison [97], one next defines the matrix valued function $\Phi$ by

$$
\Phi=\left(\begin{array}{cc}
(\operatorname{det} f)^{-1} & -(\operatorname{det} f)^{-1} \chi_{i} \\
-(\operatorname{det} f)^{-1} \chi_{i} & f_{i j}+(\operatorname{det} f)^{-1} \chi_{i} \chi_{j}
\end{array}\right) .
$$

The matrix $\Phi$ is symmetric, positive definite, and $\operatorname{det} \Phi=1$. It turns out that the vacuum field equations are equivalent to a coupled system of equations for $g_{3}=\mathrm{d} s_{3}^{2}$ and the matrix field $\Phi$, which are precisely the Euler-Lagrange equations of the action

$$
I=\int \frac{1}{2} R_{3} \star_{3} 1+\frac{1}{8} \operatorname{Tr}\left(\Phi^{-1} \mathrm{~d} \Phi \wedge \star_{3} \Phi^{-1} \mathrm{~d} \Phi\right) .
$$

Here, $R_{3}$ is the Ricci-scalar for the 3 -dimensional Lorentzian metric $g_{3}$ on $\mathbb{R} \times \hat{\mathscr{M}}$. This is the action of a 3-dimensional gravitating sigma-model. Since $\Phi$ is a real, unimodular, symmetric, positive definite $(D-2)$-dimensional matrix, it can be written as $\Phi=S^{T} S$ for some real matrix $S$ of determinant 1, i.e. an $S L(D-2)$ matrix. This matrix is defined up to $S \rightarrow R S$ for some rotation $R \in S O(D-2)$, so in this sense, $\Phi$ may be thought of as taking its values in the coset manifold $X=S L(D-2) / S O(D-2)$. The trace term in the action is precisely the standard kinetic term for the standard Riemannian metric $\mathcal{G}$ on $X$.

The equations of motion for the $\Phi$-field are equivalent to the conservation laws of the $\operatorname{dim} S L(D-2)$ currents $\mathcal{J}^{I}=\operatorname{Tr}\left(T^{I} \Phi^{-1} \mathrm{~d} \Phi\right)$, where $T^{I}$ are the generators of the Lie algebra $\mathfrak{s l}(D-2)$. We may at this stage remember that we have another Killing vector, $t$, or equivalently, that the metric $g_{3}$ does not depend on the coordinate $\tau$. Using the explicit 
form of this metric then allows one to write the equation of motion for the matrix field $\Phi$ as an equation on $\hat{\mathscr{M}}$, with metric $\mathrm{d} \hat{s}_{2}^{2}=\mathrm{e}^{-\nu}\left(\mathrm{d} r^{2}+\mathrm{d} z^{2}\right)$, i.e. an equation in $r, z$ alone. Explicitly, this is

$$
\frac{1}{r} \mathrm{~d}\left(r \star_{2} \mathcal{J}^{I}\right)=0 \quad \Longleftrightarrow \quad \partial_{z}\left(\Phi^{-1} \partial_{z} \Phi\right)+\frac{1}{r} \partial_{r}\left(r \Phi^{-1} \partial_{r} \Phi\right)=0 .
$$

The remaining Einstein equations for the sigma model action $I$ for the metric $g_{3}$ then give an equation for $\nu$. Since the equations for the currents are independent of $\nu$, it follows that the sigma-model equations are decoupled from this equation. The two sets of equations can hence be solved successively. Finally, once we have $\nu, \Phi$, we can get $A^{j}=w^{j} \mathrm{~d} \tau$ by inverting eq. (50). In this way, we can in principle solve for all unknown functions in the Weyl-Papapetrou form (39).

The sigma-model formulation of the Einstein equations has several important uses. For us, the most important one is that one can obtain "divergence identities" that play a key role in the proof of the uniqueness theorem, see the next subsection. However, another important consequence of the sigma model formulation is the possibility to generate new solutions from old ones. A simple but powerful way to do this is to apply to a $\Phi$ representing a given solution a similarity transformation $R \Phi R^{-1}$, where $R$ is any constant rotation matrix. Since such a transformation is a symmetry of the action, we get a new solution, which, because the $f_{i j}$ and $\chi_{i}$ components of $\Phi$ are mixed, will in general differ non-trivially from the original one. This type of transformation gives a group action of $S O(D-2)$ on the space of solutions. A less obvious fact is that this group can be enhanced to to the infinite-dimensional "Gerochgroup". For 4-dimensions, a the sigma-model perspective on this construction [51] may be found in [15], but the same arguments should go through in $D$-dimensional vacuum gravity as well. A related feature of the equations (53) is that solutions may be generated by the same sort of techniques ("inverse scattering method") developed originally for the Sine-Gordonand KdV-equations. For a detailed explanation how to apply such techniques, we refer to the textbook [8]. While such techniques have been widely and successfully applied to obtain highly non-trivial higher dimensional metrics, an unpleasant feature of all solution generating techniques is that, starting from a given regular, say asymptotically flat, stationary black hole solution, the newly generated solution may neither be regular, nor asymptotically flat, nor even describing a black hole.

To see more clearly the relationship between regularity and the global considerations about the orbit space made in the previous subsection, in particular the "interval structure", suppose we have a given single black hole solution characterized by a general interval structure:

\begin{tabular}{|c|c|}
\hline Interval Lengths & Vectors (Labels) \\
\hline$\infty, l_{1}, \ldots, l_{h}, \ldots, l_{N}, \infty$ & $\underline{v}_{0}, \underline{v}_{1}, \ldots, \underline{0}, \ldots, \underline{v}_{N}, \underline{v}_{N+1}$ \\
\hline
\end{tabular}

Here, $l_{h}$ corresponds to the horizon interval $\left(z_{h}, z_{h+1}\right)$ on the boundary of $(r, z)$-space. Now, recall that the integer vectors $\underline{v}_{J}$ tell us which linear combination $\sum v_{J i} \psi_{i}=0$ vanishes at 
the axis represented by the respective interval. Hence, we must have at the $J$-th interval $z \in\left(z_{J}, z_{J+1}\right)$ :

$$
\sum_{i} f_{i j}(r=0, z) v_{i J}=0
$$

Also, one can see that the potentials $\chi_{i}$ are constant along the boundary $r=0$, except at the horizon interval. The change of $\chi_{i}$ across the horizon interval can be shown to be given by

$$
\left.\frac{1}{8}(2 \pi)^{D-4} \chi_{i}(r=0, z)\right|_{z_{h}} ^{z_{h+1}}=J_{i}
$$

where $J_{i}$ is the angular momentum for the rotational plane of the $i$-th rotational Killing field $\psi_{i}$, expressible as

$$
J_{i}=\frac{1}{8 \pi} \int_{\infty} \star_{D} \mathrm{~d} \psi_{i}
$$

These conditions on $f_{i j}, \chi_{i}$, and hence $\Phi$, can be thought of as Dirichlet type boundary conditions at $r=0$. However, these boundary conditions alone do not guarantee that the corresponding spacetime metric (39) is smooth along the axis or the horizon. For this, one needs further conditions. On each interval $z \in\left(z_{J}, z_{J+1}\right)$ representing an axis, and can relatively easily see that it is required that

$$
\lim _{r \rightarrow 0} r^{-2} \mathrm{e}^{\nu(r, z)} \sum_{i, j} f_{i j}(r, z) v_{i J} v_{j J}=1
$$

to avoid a conical singularity ("strut") in the spacetime metric (39). On the horizon interval $z \in\left(z_{h}, z_{h+1}\right)$, we need instead (see Appendix of [74]):

$$
\lim _{r \rightarrow 0} \mathrm{e}^{\nu(r, z)} \operatorname{det} f(r, z)^{-1}=\kappa^{2},
$$

where $\kappa$ is the surface gravity of the horizon, again, to avoid a conical type singularity. These conditions may be thought of, coarsely speaking, as constraining the "normal derivative" of the fields $f_{i j}, \chi_{i}$, hence $\Phi$, on the boundary $r=0$. Since one is normally not free to impose both Dirichlet type and Neumann type boundary conditions simultaneously, this explains intuitively the difficulty in obtaining regular solutions via the solution generating techniques. Also note that, even if we do satisfy these conditions, they will not imply that the metric is smooth, but only that the curvature is continuous. To get smoothness, one would effectively have to control the full asymptotic expansion of the fields $\Phi$ near $r=0$. Another, related, difficulty is to control the asymptotic conditions for $r \rightarrow \infty$ in order to obtain a metric $g$ with the desired asymptotic behavior near infinity.

\subsection{Divergence identities}

The last step towards black hole uniqueness in the stationary case are "divergence identities". The idea behind such identities is actually quite simple, and is very easily explained for the 
toy example of a single scalar field, $\phi$, with strictly convex potential, $\mathcal{V}(\phi)$, on a bounded set $\Omega$ in $N$-dimensional Euclidean space $\mathbb{R}^{N}$. Such a field is described by the action

$$
S=\int_{\Omega}\left(\frac{1}{2}\left(\partial^{i} \phi\right) \partial_{i} \phi+\mathcal{V}(\phi)\right) \mathrm{d}^{N} x
$$

Consider two solutions $\phi_{1}, \phi_{2}$ to the corresponding Euler Lagrange equations and define the "current" $j^{i}=\left(\phi_{1}-\phi_{2}\right) \partial^{i}\left(\phi_{1}-\phi_{2}\right)$. Taking a divergence of the current and using the equations of motion, one has

$$
\partial_{i} j^{i}=\partial_{i}\left(\phi_{1}-\phi_{2}\right) \partial^{i}\left(\phi_{1}-\phi_{2}\right)+\left(\phi_{1}-\phi_{2}\right)\left(\mathcal{V}^{\prime}\left(\phi_{1}\right)-\mathcal{V}^{\prime}\left(\phi_{2}\right)\right) \geq 0,
$$

where the key $\geq 0$ relation follows from the fact that $\mathcal{V}^{\prime}\left(\phi_{1}\right)-\mathcal{V}^{\prime}\left(\phi_{2}\right)$ has the same sign as $\phi_{1}-\phi_{2}$ for a convex potential. Now let $n^{i}$ be the normal to $\partial \Omega$, and assume that $\phi_{1}=\phi_{2}$ on $\partial \Omega$ (Dirichlet conditions), or alternatively, that $\partial_{n} \phi_{1}=\partial_{n} \phi_{2}$ on $\partial \Omega$ (Neumann conditions). Either condition implies that $n^{i} j_{i}=0$ on $\partial \Omega$, so if we integrate the above equation over $\Omega$ and use Gauss' theorem, we get the relation

$$
0=\int_{\Omega} \partial_{i}\left(\phi_{1}-\phi_{2}\right) \partial^{i}\left(\phi_{1}-\phi_{2}\right)+\left(\phi_{1}-\phi_{2}\right)\left(\mathcal{V}^{\prime}\left(\phi_{1}\right)-\mathcal{V}^{\prime}\left(\phi_{2}\right)\right) \mathrm{d}^{N} x
$$

However, the integrand is $\geq 0$, so it must be equal to zero. Thus, we conclude that $\phi_{1}-\phi_{2}$ is a constant, and since $\mathcal{V}$ is strictly convex, that constant must vanish. Thus, the solution is unique with either Dirichlet- or Neumann boundary conditions.

In our case, we do not have a single scalar field with a convex potential, but we have a field $\Phi$ without potential valued in the curved target space $X=S L(D-2) / S O(D-2)$, satisfying the sigma-model equations given in the last section. What replaces the convexity condition on the potential $\mathcal{V}$ in this case is that the metric $\mathcal{G}$ on the target space $X$ has negative sectional curvature. There are actually several ways to take advantage of this and thereby to generalize the above divergence identity idea to this case, but they all rely, directly or indirectly, on this fact. The first construction, the so called "Mazur identity" [101], is the more explicit one, and relies on the coset structure of the target space $X$. It works as follows.

a) Mazur identity: We first define an expression representing the "difference" between the two metrics $g_{1}, g_{0}$ in the form (39), represented by the symmetric, positive, uni-modular matrices $\Phi_{1}, \Phi_{0}$. One possibility is to choose

$$
\sigma:=\operatorname{Tr}\left(\Phi_{0}^{-1} \Phi_{1}-I\right)
$$

Let $\mathcal{J}_{i}=\Phi_{i}^{-1} \mathrm{~d} \Phi_{i}$ be the matrix currents as in the previous section, and let $N$ be the matrix valued 1 -forms defined by

$$
N=S_{1}^{-1}\left(\mathcal{J}_{1}-\mathcal{J}_{0}\right)^{T} S_{0},
$$


where $S_{i}$ are real matrices such that $\Phi_{i}=S_{i} S_{i}^{T}$. Then a calculation using the equations of motion for $\Phi_{0}, \Phi_{1}$ gives Mazur's identity

$$
\frac{1}{r} \star_{2} \mathrm{~d}\left(r \star_{2} \mathrm{~d} \sigma\right)=\operatorname{Tr}\left(N^{T} \cdot N\right) \geq 0
$$

which is a divergence identity of the same nature as above, having again - very importantlya non-negative term on the right side. The domain in question is the upper half plane $\Omega=\hat{\mathscr{M}}=\{(r, z) \mid r>0\}$, with $\star_{2}$ referring to the standard flat Riemannian metric on this space. The divergence identity may now be exploited, in principle, in the same way as for the scalar field case above, by integrating it over $\Omega$. The aim is to show that the boundary term arising from the left side vanishes identically, which then immediately gives $N=0$ in $\Omega$. The last fact is furthermore relatively easily seen to give $\Phi_{1}=\Phi_{0}$, which, as is described in sec. 3.7, in turn implies that the metrics $g_{1}$ and $g_{0}$ are the same.

However, there are a number of important differences to the simple scalar field example that need to be taken into account here. These have to do with the facts that (a) the domain $\Omega$ is not compact, and (b) the singular nature of the boundary conditions to be considered at $\partial \Omega=\{r=0\}$. For point (a), one needs to understand more precisely the asymptotic conditions satisfied by $\Phi_{i}$ for $r, z \rightarrow \infty$-here asymptotic expansions need to be made - and for (b) one has to understand the behavior of $\Phi_{i}$ near the boundary $r=0$ [74]. We will discuss these issues in the next section 3.7.

Another approach, used by Bunting [12], exploits the properties of harmonic maps in negatively curved target spaces.

b) Bunting's method: Unlike Mazur's identity, this method does not use the explicit representation of $X$ as a coset manifold, but it uses only that the metric $\mathcal{G}=-\operatorname{Tr}\left(\Phi^{-1} \mathrm{~d} \Phi \otimes\right.$ $\left.\Phi^{-1} \mathrm{~d} \Phi\right)$ on $X$ is Riemannian and has negative sectional curvature. By this, one means the following. Let $\mathcal{R}_{A B C D}$ be the Riemann tensor of $\mathcal{G}_{A B}$. The Riemann tensor is always anti-symmetric in $A B$ and $C D$, and symmetric under the exchange of $A B$ with $C D$. Thus, it can be viewed as a symmetric, bilinear map Riem : $\wedge^{2} T_{\Phi} X \times \wedge^{2} T_{\Phi} X \rightarrow \mathbb{R}$ in each tangent tangent space of $X$. We say that $(X, \mathcal{G})$ has negative sectional curvature (or simply, is "negatively curved") if this bilinear form only has negative eigenvalues. In other words, there is a $\lambda>0$ such that, for any anti-symmetric 2-tensor $\omega$ we have $\operatorname{Riem}(\omega, \omega) \leq-\lambda\|\omega\|^{2}$, or in components,

$$
\mathcal{R}_{A B C D} \omega^{A B} \omega^{C D} \leq-\lambda \mathcal{G}_{A C} \mathcal{G}_{B D} \omega^{A B} \omega^{C D}
$$

This condition replaces the condition of strict convexity for the potential in the above scalar field example. It implies, but is much more stringent than, that the scalar curvature $\mathcal{R}<0$. Let $d: X \times X \rightarrow \mathbb{R}$ be the geodesic distance function on $X$, and define $d(x)=d\left(\Phi_{1}(x), \Phi_{0}(x)\right)$, $x \in \hat{\mathscr{M}}=\{(r, z) \mid r>0\}$. Then from the equations of motion (53) for $\Phi_{0}, \Phi_{1}$, there follows the inequality

$$
\frac{1}{r} \star_{2} \mathrm{~d}\left(r \star_{2} \mathrm{~d} d\right) \geq 0
$$


which is a version of the known [85] fact that the distance function between harmonic maps into a negatively curved target space is sub-harmonic. The origin of this identity can be motivated as follows. Suppose $\Phi_{t}: \Omega \rightarrow X$ is a family of solutions to the equations of motion (53), and let $\delta \Phi=\frac{\partial}{\partial t} \Phi_{t}: \Omega \rightarrow \Phi_{t}^{*} T X$ be the linearization at any fixed $t$, interpreted as the infinitesimal displacement of the 2-dimensional "worldsheet" in $X$ swept out by $\Phi_{t}$. Then, one can derive from the sigma-model field equation satisfied by each $\Phi_{t} \equiv \Phi$ the following equation for $\delta \Phi_{t} \equiv \delta \Phi$ :

$$
\frac{1}{r} \star_{2} \nabla\left(r \star_{2} \nabla \delta \Phi^{A}\right)=\mathcal{R}_{B C D}^{A}(\Phi)\left(\mathrm{d} \Phi^{B}\right) \cdot\left(\mathrm{d} \Phi^{D}\right) \delta \Phi^{C} .
$$

Here $\nabla$ is the natural derivative operator in the bundle $\Phi^{*} T X \rightarrow \Omega$ that is inherited from the derivative operator of the metric $\mathcal{G}$ on $X$. This equation may be viewed as the generalization of the "geodesic deviation equation" in $X$ from curves to surfaces, with $t \mapsto \Phi_{t}$ playing the role of a 1-parameter family $c_{t}(\lambda)$ of geodesic curves in $X$ interpolating between two curves $c_{0}, c_{1}$, with $\delta \Phi_{t}$ playing the role of the displacement vector field $v_{t}=\delta c_{t}=\frac{\partial}{\partial t} c_{t}$ of the family, with $\mathrm{d} \Phi_{t}$ playing the role of $\mathrm{d} c_{t} / \mathrm{d} \lambda$, and with $\Omega$ playing the role of the proper length parameterization of the curve $c(\lambda)$ :

$$
\frac{\mathrm{d}^{2}}{\mathrm{~d} \lambda^{2}} v^{A}=\mathcal{R}_{B C D}^{A}(c) \frac{\mathrm{d} c^{B}}{\mathrm{~d} \lambda} \frac{\mathrm{d} c^{D}}{\mathrm{~d} \lambda} v^{C}
$$

Now assume that $[0,1] \ni t \mapsto \Phi_{t}$ connects $^{9}$ two given solutions $\Phi_{0}, \Phi_{1}$ of the sigma model equations. Define

$$
s\left(\Phi_{0}, \Phi_{1}\right)(x):=\int_{0}^{1}\left(\mathcal{G}_{A B}\left(\Phi_{t}\right) \delta \Phi_{t}^{A} \delta \Phi_{t}^{B}\right)(x) \mathrm{d} t .
$$

Then it is straightforward to derive from eq. (67) the following formula

$$
\begin{aligned}
& \frac{1}{r} \star_{2} \mathrm{~d}\left(r \star_{2} \mathrm{~d} s\right) \\
= & \int_{0}^{1} \mathrm{~d} t\left(\mathcal{G}_{A B}\left(\Phi_{t}\right) \nabla \delta \Phi_{t}^{A} \cdot \nabla \delta \Phi_{t}^{B}-\mathcal{R}_{A B C D}\left(\Phi_{t}\right)\left(\delta \Phi_{t}^{A} \mathrm{~d} \Phi_{t}^{C}\right) \cdot\left(\delta \Phi_{t}^{B} \mathrm{~d} \Phi_{t}^{D}\right)\right) \\
\geq & \int_{0}^{1} \mathrm{~d} t\left(\mathcal{G}_{A B} \nabla \delta \Phi_{t}^{A} \cdot \nabla \delta \Phi_{t}^{B}+\lambda \mathcal{G}_{A B} \mathcal{G}_{C D}\left(\delta \Phi_{t}^{[A} \mathrm{d} \Phi_{t}^{C]}\right) \cdot\left(\delta \Phi_{t}^{[B} \mathrm{d} \Phi_{t}^{D]}\right)\right) \geq 0,
\end{aligned}
$$

where to get the key $\geq 0$ relations we have used (a) that the target space is Riemannian, and (b) that it is negatively curved. Note the close analogy between this equation, and the Mazur identity (64), and (66). Either identity can be used to show that the solutions $\Phi_{0}, \Phi_{1}$ are equal, by integrating over $\Omega$. Then, if one can show that the surface term from the left side vanishes, so does $\nabla \delta \Phi_{t}^{A}$, from which one then concludes that $\delta \Phi_{t}=0$, i.e. $\Phi_{t}$ is independent of $t$ and hence $\Phi_{0}=\Phi_{1}$.

\footnotetext{
${ }^{9}$ The implicit assumption is that the space of solutions is connected. This is not required for (66).
} 
However, note that the method based on (66) is potentially more generally applicable than Mazur's identity because it only uses that $X$ is a negatively curved manifold, whereas Mazur's identity essentially uses the fact that $X=S L(D-2) / S O(D-2)$ is a symmetric space of "non-compact type" ${ }^{10}$. While this is a sufficient condition for $X$ to be negatively curved by general results of symmetric spaces of non-compact type [65], it is not a necessary condition. It can be very complicated, and requires considerable creativity, to find a cosetspace parameterization of this nature for the target space $X$ (see below sec. (4)) in more general Einstein-matter systems. On the other hand, it is essentially a mechanical task to check whether or not the target space metric is negatively curved. Thus, it appears that Bunting's method has some potential advantage of Mazur's method in this regard.

\subsection{Uniqueness theorems for rotating stationary black holes}

We can now combine the results in the previous subsections and state a uniqueness theorem for higher dimensional rotating, stationary black holes in vacuum general relativity. To prove this uniqueness theorem, one needs to assume the existence of $D-3$ Killing fields $\psi_{1}, \ldots, \psi_{D-3}$ generating an isometric action of $U(1)^{D-3}$ on the spacetime. Note that this is very likely non-generic; the rigidity theorem is consistent with having only a single $U(1)$ factor. One also assumes that the spacetime is asymptotically Kaluza-Klein, in the sense described in sec. 3.1.

Theorem 10. ("Uniqueness theorem for rotating Kaluza-Klein black holes" [74]) There can be at most one stationary, single horizon, vacuum, non-extremal, asymptotically KaluzaKlein spacetime $(\mathscr{M}, g)$ with $s=3,4$ or 5 large dimensions, with $D-3$ axial Killing fields, satisfying the technical assumptions stated in sec. 3.1, for a given interval structure $\left\{\underline{v}_{I}, l_{I}\right\}$ and a given set of angular momenta $\left\{J_{i}\right\}, i=1, \ldots, D-3$.

This theorem is essentially the only uniqueness theorem of this generality known for stationary black hole solutions in higher dimensions, although the argument can presumably be generalized to other theories of the type described below in sec. 4. For antecedents of the theorem in 5-dimensions see [106] (trivial topology and horizon $S^{3}$ ) and [73] (arbitrary interval structure). It is not hard to generalize the theorem to black holes with multiple horizons. In that case, one has to specify separately the angular momenta $J_{i}\left(B_{j}\right)=1 / 8 \pi \int_{B_{j}} \star_{D} \mathrm{~d} \xi_{i}$ for each connected component of the horizon.

In $D=4$ with no extra dimensions, the only non-trivial interval structure for a single black hole spacetime is given by the intervals $\left(-\infty,-z_{0}\right],\left[-z_{0}, z_{0}\right],\left[z_{0}, \infty\right)$. The middle interval corresponds to the horizon, while the half-infinite ones to the axis of the rotational Killing field. The interval vectors $\left\{\underline{v}_{I}\right\}$ are 1-dimensional integer vectors in this case and

\footnotetext{
${ }^{10}$ By this one means a triple $(G, H, \tau)$, where $\tau$ is an involution on the non-compact Lie-group $G$, and where $H$ is a maximally compact subgroup invariant under $\tau$. In the present example, $\tau$ is given by $\tau(g)=\left(g^{-1}\right)^{T}$. Mazur's identity can be generalized to any such symmetric space, as described in the original paper [101].
} 
hence trivial. For each $z_{0}>0$ and for each angular momentum $J$, there exists precisely one solution given by the appropriate member of the Kerr-family of metrics. Thus, one finds that the Kerr metrics exhaust all possible stationary, axially symmetric single black hole spacetimes (satisfying the technical assumptions stated in sec. 3.1). This is of course just the classical uniqueness theorem for the Kerr-solution [12, 17, 101, 119, 62], see [20, 21] for a coherent exposition straitening several technical issues [see also [21]]. The mass $m$ of the non-extremal Kerr solution characterized by $z_{0}, J$ is related to these parameters by $z_{0}=\sqrt{m^{2}-J^{2} / m^{2}}>0$. Hence the uniqueness theorem could be stated equivalently in terms of $m$ and $J$, which is more commonly done. Note that the length of the horizon interval, $l_{h}=2 z_{0}$ tends to zero in the extremal limit, in accordance with (71).

In higher dimensions, one may similarly derive relations between the interval structure and angular momenta on the one side, and the other invariants on the other side for any given solution. For example, the relation between the length of the horizon interval $l_{h}$, the horizon area $A_{h}$, and the surface gravity is always [74]

$$
(2 \pi)^{D-3} l_{h}=\kappa A_{h}
$$

Other formulae of this nature are provided for the Myers-Perry or black-ring solutions e.g. in [59], but they are not expected to be universal. Of course, for most interval structures it is not known whether there actually exists a solution, so in this sense much less is known in higher dimensions than in $D=4$.

Outline of proof of thm. 10: We have already presented individually most of the pieces of the argument, and we now put them together. Suppose one has two spacetimes $\left(\mathscr{M}_{1}, g_{1}\right),\left(\mathscr{M}_{2}, g_{2}\right)$ as in the theorem. Since the interval structures determine the topology as well as the nature of the action of the isometries by the "orbit space theorem", these must coincide for both spacetimes, so $\mathscr{M}_{1}=\mathscr{M}_{2}$. Next, by the "Weyl-Papapetrou theorem", one can introduce coordinates (39) in both spacetimes, which give rise to sigma model fields $\Phi_{1}, \Phi_{2}$ on the upper half plane $\{(r, z) \mid r>0\}$. Next one uses the Mazur identity (64). It is technically convenient to rewrite this equation as follows. Introduce an auxiliary $\mathbb{R}^{3}$ with coordinates $(x, y, z)$, related to the upper half space coordinates by $(x, y, z)=(r \cos \varphi, r \sin \varphi, z)$. Then, view $\Phi_{i}$ as cylindrically symmetric functions on this $\mathbb{R}^{3} \backslash\{r=0\}$. The Mazur identity implies

$$
\left(\partial_{x}^{2}+\partial_{y}^{2}+\partial_{z}^{2}\right) \sigma \geq 0
$$

The key point is now to understand the behavior of $\sigma$, eq. (62), near the "z-axis" $\{r=0\}$ of $\mathbb{R}^{3}$. Here, one uses again the interval structure, together with the boundary conditions (54), and the information about the angular momenta, together with the jump relation (55). One can show from this that $\sigma$ is uniformly bounded. That part of the analysis is actually by far the most tedious one, since $\sigma$ can have a priori very direction-dependent limits along the $z$-axis, due to the fact that the Weyl-Papapetrou coordinates $r, z$ give a very distorted perspective on the true spacetime geometry there. Therefore these limits, and that at infinity, 
need to be investigated with a certain amount of care, but in the end one finds that $\sigma$ is uniformly bounded. It also follows from the definition (62) that $\sigma \geq 0$. Now one can apply Weinstein's lemma [133], which is a version of the maximum principle:

Lemma 1. ("Weinstein's lemma") Let $\sigma(x, y, z) \geq 0$ be a continuous function on $\mathbb{R}^{3} \backslash\{r=0\}$ which is a solution to $\left(\partial_{x}^{2}+\partial_{y}^{2}+\partial_{z}^{2}\right) \sigma \geq 0$, in the distributional sense, and which is uniformly bounded by a constant. Then $\sigma=0$.

Once we know that $\sigma=0$, we can derive that $\Phi_{1}=\Phi_{2}$, and that the remaining functions $\nu, w^{i}$ in the Weyl-Papapetrou form also coincide for both metrics.

\subsection{Uniqueness for non-rotating (static) black holes}

While the uniqueness theorem for rotating higher dimensional black holes given in the previous subsection required by-hand assumptions about the isometry group which are most likely not generic, the situation is much better with regard to non-rotating stationary black holes, as we will explain in this subsection for the case of vacuum relativity.

First, in the non-rotating case, one can apply the staticity theorem of Sudarsky and Wald [126], which straightforwardly generalizes to any dimension:

Theorem 11. ("Staticity theorem") Let $(\mathscr{M}, g)$ be a smooth, asymptotically flat nonrotating, non-extremal black hole satisfying the vacuum Einstein equations. Then $(\mathscr{M}, g)$ is in fact static, i.e. the Killing vector field $t$ is surface orthogonal $t \wedge \mathrm{d} t=0$.

Remark: Versions of the staticity theorem also exist in other theories such as EinsteinMaxwell-Dilaton [123], and also to Einstein-Yang-Mills theory under certain assumptions about the electric/magnetic charges of the fields [126].

Once one knows that the spacetime is static, one can analyze its uniqueness properties by a method that is entirely different from reduction to a sigma-model described in sec. 3.5. This method is more powerful-albeit restricted to the static case-in that one does not have to know, a priori, that the spacetime has any other symmetries apart from time-translations, and one can also completely bypass the topology and rigidity arguments that are a prerequisite in the stationary case. The theorem is $[77,54,53]$ :

Theorem 12. ("Uniqueness of static black holes") An asymptotically flat (in the standard sense) static, vacuum black hole with non-degenerate event horizons in any dimensions $D \geq 4$ is isometric to the Schwarzschild-Tangherlini metric.

Remark: In 4-dimensions, one can remove the condition that the event horizon has no degenerate components [24]. For the higher dimensional case, the non-existence of such a static, vacuum degenerate black holes has been discussed [24] but not proven yet. 
The theorem has been established first in $D=4$ by Israel [84]. Later, Bunting and Masoodul-Alam [13], invented an ingenious method based on a clever use of the positive mass theorem. However, both proofs used some geometric properties that hold only in 4-dimensions; the proof by [84] applies the Gauss-Bonnet theorem, while the proof of [13] uses special properties of the Weyl-Bach tensor in low dimensions. It is therefore not a straightforward task to generalize the proof of either [84] and/or [13] to higher dimensions. The proof by [77, 52] is based on [13], and it bypasses the use of the Weyl-Bach tensor using properties of special surfaces in $\mathbb{R}^{n}$.

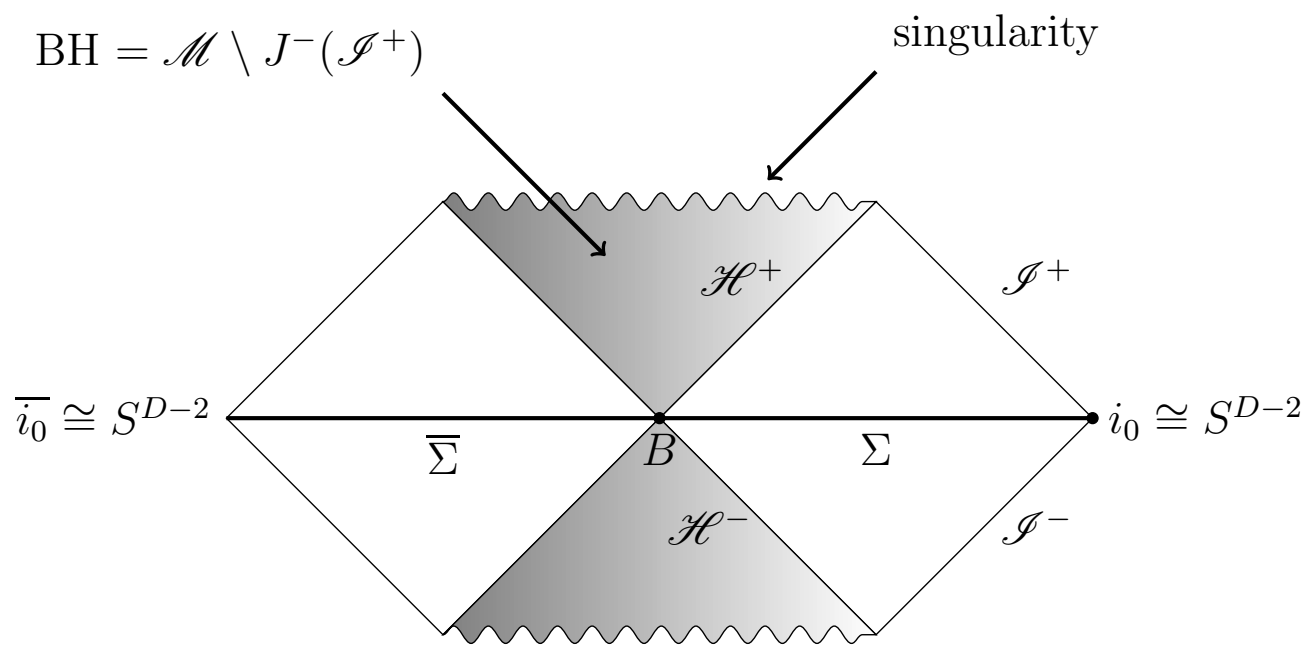

That proof may be summarized as follows (see also [78]):

1. First, one constructs a complete Riemannian manifold $\tilde{\Sigma}$ with an asymptotically flat metric $\tilde{h}$. This is done by taking a spatial slice $(\Sigma, h)$ of the spacetime, eq. (5), orthogonal to the orbits of the Killing vector field, $t=\partial / \partial \tau$, with $\partial \Sigma=B$, and then doubling $(\Sigma, h)$ across the horizon cross section $B$ and gluing a copy $\bar{\Sigma}$ onto $\Sigma$ along $B$. Then, one performs a conformal rescaling from $h$ to $\tilde{h}=\Omega^{2} h$ on the doubled spacetime $\tilde{\Sigma}=\Sigma \cup \bar{\Sigma}$, with $\Omega$ chosen in such a way that $\Omega \rightarrow 1$ near the spatial infinity $i_{0}$ of $\Sigma$, and $\Omega \rightarrow 0$ near the spatial infinity $\bar{i}_{0}$ of $\bar{\Sigma}$, and adds a single point to $\bar{\Sigma}$ to cap off $\bar{i}_{0}$. Hence, the slice $\tilde{\Sigma}$ only has one asymptotic end, $i_{0}$, see the above figure.

2. The next step is to show that $(\tilde{\Sigma}, \tilde{h})$ is flat Euclidean space. This is done by an appropriate choice of the conformal factor $\Omega$, the asymptotic flatness of the original metric $h$, and the use of the positive energy theorem. To make this work, $\Omega$ must be chosen to make the scalar curvature $\tilde{R}$ of $\tilde{h}$ non-negative. To start, note that the condition of the asymptotic flatness implies:

$$
\begin{aligned}
N & =1-\frac{M}{r^{D-3}}+O\left(r^{-(D-2)}\right) \\
h & =\left(1+\frac{2}{D-3} \frac{M}{r^{D-3}}\right) \delta+O\left(r^{-(D-2)}\right)
\end{aligned}
$$


as in eq. (5). Now, choose the conformal factor $\Omega=\left\{\Omega_{+}\right.$on $\Sigma, \Omega_{-}$on $\left.\bar{\Sigma}\right\}$ by

$$
\Omega_{ \pm}=\left(\frac{1 \pm N}{2}\right)^{2 /(D-3)} .
$$

Then, asymptotic flatness, eq. (74), translates in terms of the rescaled metric $\tilde{h}$ into

$$
\tilde{h}=\delta+O\left(r^{-(D-2)}\right)
$$

implying, very importantly, that the mass $\tilde{M}$ of $(\tilde{\Sigma}, \tilde{h})$ is zero. One can also check that with the choice eq. (75), the scalar curvature of $\tilde{h}$ on $\tilde{\Sigma}$ is non-negative, and that it is sufficiently regular across $B$ in order to be able to apply the positive mass theorem [134, 125, 57], which states that:

Theorem 13. Consider an $n$-dimensional, complete, $\operatorname{spin}^{11}$, Riemannian manifold, $(\tilde{\Sigma}, \tilde{h})$ with asymptotically flat end, i.e., Euclidean outside a compact set and the metric decays as in eq. (74). If the scalar curvature of $\tilde{h}$ is non-negative, then the ADM mass of $(\tilde{\Sigma}, \tilde{h})$ is also non-negative. Furthermore, if the ADM mass vanishes, then $(\tilde{\Sigma}, \tilde{h})$ is isomorphic to flat space $\left(\mathbb{R}^{n}, \delta\right)$.

By the positive mass theorem, we conclude that $\tilde{h}$ is flat, and hence that $h$ is conformally flat, i.e. $h=\Omega^{-2} \delta$, and we also conclude that $\tilde{\Sigma} \cong \mathbb{R}^{D-1}$, i.e., $\Sigma \cong \mathbb{R}^{D-1} \backslash \mathrm{BH}$, where $\mathrm{BH}$ is a compact manifold with boundary $B$. We also know by construction that the lapse function $N$ is determined by the conformal factor $\Omega$ via eq. (75). Thus, $(\Sigma, h, N)$ will be unique if we can show that $\Omega$ is uniquely specified.

3. One shows that $\Omega$ is unique. Introducing $u_{ \pm}:=\Omega_{ \pm}^{-1}$, one shows that the Laplace equation holds on the flat base space $\mathbb{R}^{D-1}$,

$$
\Delta u_{ \pm}=0
$$

where $\Delta$ is the ordinary flat space Laplacian for the metric $\delta$. The boundary condition at infinity $r \rightarrow 0$ follows from the conditions of asymptotic flatness on $(\Sigma, N, h)$, eq. (74). The boundary condition at $B$ follows from the fact that $B$ represents a horizon, eq. (75), which gives $N=0$ on $B$. These conditions together with the Laplace equation uniquely determine $u_{ \pm}$, hence $\Omega$, once we know exactly what the nature of the boundary $B$ in our flat base space $\mathbb{R}^{D-1}$ is.

\footnotetext{
${ }^{11}$ The requirement that $\tilde{\Sigma}$ be spin is not needed in dimensions $n=3$ because the technique of [134] does not require spinors. A generalization of that argument to dimensions less than $n=8$ is also available, so up to that dimensions, we do not need a spin structure. In higher dimensions, the proof of the theorem is based on the Witten spinor method, and therefore only works for spin manifolds.
} 
4. The final step is to show that the inner boundary $B$ is a spherically symmetric hypersurface in the base space $\mathbb{R}^{D-1}$. It is then straightforward to show that the unique solutions $h$ and $N$ coming from eq. (77), which also must respect the spherical symmetry, in fact correspond to the lapse function and the spatial part of the Schwarzschild metric in isotropic coordinates. At this point, it becomes relevant what the spacetime dimension is. In the 4-dimensional case, spherical symmetry can be shown using the fact that Weyl-tensor of 3-dimensional space vanishes. This argument does not work in the case in $D-1 \geq 4$. However, in any dimension, the vacuum Einstein equation implies that the lapse function $N$ is harmonic on $(\Sigma, h)$, from which one can argue, using the maximum principle, that it is possible to take $N$ as a coordinate, so that

$$
h=\rho^{2} \mathrm{~d} N^{2}+\gamma_{a b} \mathrm{~d} x^{a} \mathrm{~d} x^{b}
$$

where the function $\rho$ has been introduced so that the one-form $\rho \mathrm{d} N$, normal to each $N=$ const. level surface with the metric $\gamma_{a b}$, is normalized with respect to the metric $h$, and the trajectory of the coordinates $x^{a}$ on level sets of $N$ are orthogonal to each level set. The level set $N=0$ corresponds to the bifurcation surface $B$ of the horizon. The regularity of $g$ and the bifurcate surface property implies that $B$ must be totally geodesic in $\Sigma$, i.e., the extrinsic curvature $k$ of $B$ as an embedded $(D-2)$-hypersurface in $\Sigma$ must be zero. Using this result, one can show that the conformally transformed

$B$ viewed as a surface in $(\tilde{\Sigma}, \delta)$ must be totally umbillic, i.e., $\tilde{k}_{a b} \propto \tilde{\gamma}_{a b}$. Then, one can appeal to a well-known mathematical result that a totally umbilical embedding of $(n-1)$-surface into $n$-dimensional Euclidean space is spherical [88]. Thus, each connected component of the horizon $B$ has been shown to be spherically symmetric. One can also show that $B$ must be connected, therefore $B$ is metrically a sphere in $\left(\mathbb{R}^{D-1}, \delta\right)$. As we have already seen, this implies that $(N, h)$ coincide with the corresponding quantities in the Schwarzschild spacetime.

\subsection{Extremal black holes and their near horizon geometries}

The uniqueness theorems discussed so far make the assumption that all components of the event horizon be non-degenerate. There are, however, a number of known exact solutions with degenerate (extremal) event horizon. It is interesting to know to what extent these solutions are unique.

a) Rotating case : The uniqueness proof for rotating black holes proceeds, as in the non-

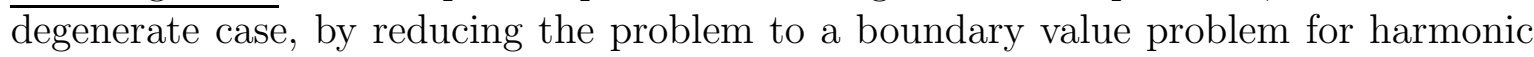
maps, using either Mazur's or Bunting's method. As in the non-degenerate case, the last step is to analyze boundary conditions for those harmonic maps on the twodimensional orbit space $\hat{\mathscr{M}}=\{(r, z) \mid r>0\}$. One of the boundary conditions is imposed at the horizon interval on the boundary $r=0$. However, a new feature arises when the horizon is the degenerate type, because the corresponding interval shrinks 
to a single point according to (71). This means basically that the Weyl-Papapetrou coordinate system is very ill-adapted to resolve the geometry near the horizon, and some sort of "blow up" is required at this point in order to analyze the boundary behavior for the harmonic maps there.

In 4-dimensions, uniqueness theorems for extremal Kerr and extremal charged Kerr black holes have been shown recently by [23, 3, 37]. The key new element in these proofs is a uniqueness result $[58,96,92]$ that the near-horizon geometry for a degenerate Killing horizon of any stationary axisymmetric vacuum spacetimes with given mass and angular momentum must agree with that of the extremal Kerr metric [7]. The near-horizon geometry is obtained by an infinite scaling of the horizon neighborhood, which, loosely speaking, achieves the desired blow up at the horizon, and which makes it possible to understand the relevant boundary conditions for the sigma-model fields at the degenerate horizon. (A more detailed description of this scaling is given below.) A similar uniqueness result also holds for the extremal electrovacuum black hole case. It is possible also in $D \geq 5$ dimensions to classify the near horizon geometries under the type of symmetry assumption as in our uniqueness theorem 10, see below. However, these results alone do not suffice for a proof of an analogue of our uniqueness theorem 10 in general dimension, because it is unclear in general dimension how the parameters of the weighted orbit space, $\left\{l_{J}\right\}$ and $\left\{\underline{v}_{J}\right\}$, are related to the parameters of the near horizon geometries. A result in this direction in $D=5$ is Theorem 2 of Ref. [37], stating that the interval structure can be used to uniquely determine extremal vacuum black holes in 5-dimensions, (as well as near-horizon geometries) under similar assumptions of the uniqueness theorem of [73]. In general dimensions, however, this remains an open problem.

b) Static case : As shown in $[19,24]$, there are no asymptotically flat, static vacuum black holes with degenerate components of the event horizon. This analysis has been generalized to include Maxwell field [25] by using properties of the near-horizon geometry. It was shown that the Majumdar-Papapetrou spacetime is the only asymptotically flat, static electro-vacuum black hole spacetime with degenerate components of the event horizon [24]. See also Ref. [121, 122] for related work. Static near-horizon geometries in 5-dimensional Einstein-Maxwell theory with Chern-Simons term have been classified [93].

c) Supersymmetric black holes are extremal and their classification is discussed below in sec. 4.

As we have explained in (a), the concept of near horizon geometry has a potential significance in proving uniqueness of higher dimensional black holes in a situation wherein one has the amount of symmetry described in our uniqueness theorem 10. However, as we have also emphasized several times already, these symmetry assumptions are most likely non-generic 
(other than in $D=4$ ). Therefore, as a stepping stone towards a classification of extremal black holes having less symmetry, one might at first look at the corresponding classification of their near horizon geometries, which are simpler, i.e., more symmetric. Let us therefore describe what a near horizon geometry actually is, what the corresponding equations are, etc.

Near Horizon Geometries: Let $(\mathscr{M}, g)$ be an extremal vacuum black hole spacetime with Killing horizon $\mathscr{H}$, and Killing vector field $K$ tangent to the null-generators of $\mathscr{H}$. (In the non-extremal case, the existence of $K$ automatically follows from the stationarity assumption by the rigidity theorem, in the extremal case, the rigidity theorem is not quite as powerful as yet, see the discussion in sec. 3.2.) Now write the metric near $\mathscr{H}$ in Gaussian null-coordinates as in eq. (14). As argued there, we may assume that, in Gaussian null coordinates, $K=$ $\partial / \partial u=n$. Then, none of the tensor fields $\alpha, \beta, \gamma$ in (14) depend on $u$, but only on $r$ and the coordinates $x^{a}$ on the horizon cross section $B$. From eq. (15), and the definition of the surface gravity, eq. (7), it follows that $\alpha=0$ on $\mathscr{H}$ in the extremal case. Hence, $\alpha$ can be written as $r$ times a smooth function, which by abuse of notation we shall call $\alpha$ again. In summary, in the extremal case, the metric in an open neighborhood of a Killing horizon $(r=0)$ can be assumed to take the form

$$
g=2 \mathrm{~d} u\left(\mathrm{~d} r-r^{2} \alpha \mathrm{d} u-r \beta_{a} \mathrm{~d} x^{a}\right)+\gamma_{a b} \mathrm{~d} x^{a} \mathrm{~d} x^{b} .
$$

None of the tensor fields depend on the coordinate $u$. Consider now for small $\epsilon>0$ the diffeomorphism $\phi_{\epsilon}$ mapping a point with coordinates $\left(r, u, x^{a}\right)$ to the point with rescaled coordinates $\left(\epsilon r, u / \epsilon, x^{a}\right)$. The near horizon limit $g_{0}$ is defined by the limit, as $\epsilon \rightarrow 0$, of the family of metrics $g_{\epsilon}=\phi_{\epsilon}^{*} g$. It is concretely given by the same formula as (79), but with the tensor fields $\alpha, \beta, \gamma$ replaced by their restriction to the horizon cross section $B$, i.e. $r=0$. In other words, these tensor fields now no longer depend on $r$ (and not on $u$ either). By construction, the near horizon limit is a vacuum solution. The infinite rescaling means that Einstein's equations reduce to the lowest order in $r$ contribution to eqs. (3.2) (and the remaining components). Concretely, they are in the vacuum

$$
\begin{aligned}
& 0=R_{a b}(\gamma)-\frac{1}{2} \mathscr{L}_{\beta} \gamma_{a b}-\frac{1}{2} \beta_{a} \beta_{b}, \\
& 0=2 \alpha+\frac{1}{2} \beta_{a} \beta^{a}+\frac{1}{2} D_{a} \beta^{a} .
\end{aligned}
$$

Thus, the near horizon geometry limit has drastically reduced the complexity of the field equations: we 'just' have to solve the above two equations for the tensor fields $\alpha, \beta, \gamma$ on the compact $(D-2)$-dimensional horizon cross section manifold $B$. Furthermore, $\alpha$ is absent from the first equation, which we may therefore solve first, and then trivially use the second equation to determine $\alpha$. 
Unfortunately, it is still by no means a simple task to solve the near horizon equations, and a complete classification of solutions for an arbitrary compact manifold $B$ (or at least a class of such manifolds) is not available at present. Besides the known solutions that can be obtained from concrete extremal black holes such as Myers-Perry, an infinite class of solutions was recently obtained by [94] for even dimensions $D$. These authors use an ansatz for $\gamma, \beta$ in terms of a $(D-4)$-dimensional compact Kähler-Einstein base space (inspired by the concrete form of the near horizon Myers-Perry solution), which is the basic input into their metrics. The near-horizon equations can then be solved by integrating certain ordinary differential equations. Since a wide variety of compact, even-dimensional Kähler-Einstein manifolds is known, this produces a correspondingly wide class of solutions to the near horizon equations (80), some of which have a very low amount of symmetry (the authors also admit a cosmological constant, suppressed in (80)). Consequently, for most of their solutions, it is not known whether they actually arise from a full-fledged extremal black hole, or not.

Another approach to the classification of near horizon geometries is to assume, from the outset, a comparable amount of symmetry as in our uniqueness theorem for non-extremal black holes, thm. 10. Thus, we assume by hand the existence of $D-3$ additional Killing fields $\psi_{1}, \ldots, \psi_{D-3}$ generating an isometric action of $U(1)^{D-3}$ on spacetime. We may assume that these Killing fields are tangent to the cross section manifold $B$, so $\alpha, \beta, \gamma$ are Lie-derived by these Killing fields. What is the total isometry group of such a near horizon metric? From the outset, we assumed the Killing field $K=\partial / \partial u$, but, after the near horizon limit, we obtain the additional Killing field $X=-u \partial / \partial u+r \partial / \partial r$ which generates the rescalings $\phi_{\epsilon}$. Together, $K, X$ generate a 2 -dimensional Lie-group $G_{2}$ which is isomorphic to the semi-direct product of translations and dilatations of the real line. So, the total, manifest, isometry group of (79) with our assumptions is $G_{2} \times U(1)^{D-3}$.

It is surprising that Einstein's equations imply that the full isometry group is actually even bigger and in fact enhanced to $S L(2) \times U(1)^{D-3}$, where $S L(2)$ contains $G_{2}$ [91]. The origin of this symmetry enhancement can be explained as follows. Letting $f_{i j}=g\left(\psi_{i}, \psi_{j}\right)$ as above, a coordinate $-1 \leq x \leq 1$ is introduced by demanding the metric $\gamma$ can be written as

$$
\gamma=\frac{1}{C^{2} \operatorname{det} f} \mathrm{~d} x^{2}+f_{i j}(x) \mathrm{d} \varphi^{i} \mathrm{~d} \varphi^{j}
$$

where $C>0$ is a constant. Geometrically, $x$ is a coordinate parameterizing the orbit space $B / U(1)^{D-3} \cong[-1,1]$. The $2 \pi$-periodic coordinates $\varphi^{i}$ have also been introduced in such a way that $\psi_{i}=\partial / \partial \varphi^{i}$. It is next shown that there is a function $\lambda$ on $B$ (effectively of $x$ ) such that

$$
\beta=\mathrm{d} \lambda+C \mathrm{e}^{\lambda} k_{i} \mathrm{~d} \varphi^{i}
$$

where we have introduced the scalar functions

$$
k_{j}:=C^{-1} \mathrm{e}^{-\lambda} i_{\psi_{j}} \beta .
$$


The next coordinate, $\rho$, is defined by

$$
\rho:=r \mathrm{e}^{\lambda},
$$

and we keep $u$ as the last remaining coordinate. The coordinates $\left(\varphi^{i}, \rho, x, u\right)$ are the desired geometrical coordinates. The Einstein equations now impose relations between the various functions $k_{j}, \alpha, \lambda, f_{i j}$, [91]. Namely, one finds that $k^{i}=f^{i j} k_{j}$ are simply constants, and that $2 \alpha \mathrm{e}^{-\lambda}-\mathrm{e}^{\lambda} k_{i} k^{i}$ is a negative ${ }^{12}$ constant, which one may choose to be $-C^{2}$ after a suitable rescaling of the coordinates $\rho, u$ and the constants $k^{i}$, and by adding a constant to $\lambda$. The Einstein equations are seen to further imply that $\mathrm{e}^{-\lambda}=\left(1-x^{2}\right)(\operatorname{det} f)^{-1}$. These conditions then imply together that the near horizon metric is given by

$$
g_{0}=\frac{1-x^{2}}{\operatorname{det} f(x)}\left(2 \mathrm{~d} u \mathrm{~d} \rho-C^{2} \rho^{2} \mathrm{~d} u^{2}\right)+\frac{\mathrm{d} x^{2}}{C^{2} \operatorname{det} f(x)}+f_{i j}(x)\left(\mathrm{d} \varphi^{i}+\rho C k^{i} \mathrm{~d} u\right)\left(\mathrm{d} \varphi^{j}+\rho C k^{j} \mathrm{~d} u\right)
$$

where $k^{i}, C$ are constants. The $S L(2)$ symmetry now arises because the first term in parenthesis is just the metric of $A d S_{2}$, with isometry group $S L(2)$ [91]. The $A d S_{2}$-factor plays a crucial role in string theory analyses of black hole entropy of extremal black holes.

The functions $f_{i j}(x)$ and their relationship to the constants $k^{i}, C$ still have not been determined, but it turns out that this can also be done, making use of the sigma-model formulation explained in sec. 3.5 (the coordinates $x, \rho$ turn out to be closely related to the Weyl-Papapetrou coordinates $r, z$ in thm. 8). This final step was carried out in $D$-dimensions in [70], using earlier work of refs. [92, 93, 36].

To state the result, we must remember that, by the orbit space theorem 9 , at each point $x= \pm 1$, there exists an integer linear combination of the vectors $\psi_{i}$ which vanishes. The coefficients in these two integer linear combinations make up two vectors $\underline{a}_{+}:=\underline{v}_{h-1}, \underline{a}_{-}:=$ $\underline{v}_{h+1} \in \mathbb{Z}^{D-3}$, which are the "winding numbers" of the intervals adjacent to the horizon interval in the orbit space theorem. The classification theorem is then [70]:

Theorem 14. All smooth, non-static, near horizon metrics with $D-3$ commuting Killing fields generating $U(1)^{D-3}$ are parametrized by real parameters $c_{ \pm}, \mu_{i}, s_{I i}$, and the integers $a_{ \pm}^{i}$ where $I=0, \ldots, D-5$ and $i=1, \ldots, D-3$, and g.c.d. $\left(a_{ \pm}^{i}\right)=1$. The explicit form of the near horizon metric in terms of these parameters is

$$
\begin{aligned}
g_{0}= & \mathrm{e}^{-\lambda}\left(2 \mathrm{~d} u \mathrm{~d} \rho-C^{2} \rho^{2} \mathrm{~d} u^{2}+C^{-2} \mathrm{~d} \theta^{2}\right)+\mathrm{e}^{+\lambda}\left\{\left(c_{+}-c_{-}\right)^{2}\left(\sin ^{2} \theta\right) \Omega^{2}\right. \\
& +(1+\cos \theta)^{2} c_{+}^{2} \sum_{I}\left(\omega_{I}-\frac{s_{I} \cdot a_{+}}{\mu \cdot a_{+}} \Omega\right)^{2}+(1-\cos \theta)^{2} c_{-}^{2} \sum_{I}\left(\omega_{I}-\frac{s_{I} \cdot a_{-}}{\mu \cdot a_{-}} \Omega\right)^{2} \\
& \left.+\frac{c_{ \pm}^{2} \sin ^{2} \theta}{\left(\mu \cdot a_{ \pm}\right)^{2}} \sum_{I<J}\left(\left(s_{I} \cdot a_{ \pm}\right) \omega_{J}-\left(s_{J} \cdot a_{ \pm}\right) \omega_{I}\right)^{2}\right\} .
\end{aligned}
$$

\footnotetext{
${ }^{12}$ Here one must use that the metric is not static, i.e. that not all $k^{i}$ vanish.
} 
Here, $x=\cos \theta$, the sums run over $I, J$ from $0, \ldots, D-5$, the function $\lambda(\theta)$ is given by

$$
\exp [-\lambda(\theta)]=c_{+}^{2}(1+\cos \theta)^{2}+c_{-}^{2}(1-\cos \theta)^{2}+\frac{c_{ \pm}^{2} \sin ^{2} \theta}{\left(\mu \cdot a_{ \pm}\right)^{2}} \sum_{I}\left(s_{I} \cdot a_{ \pm}\right)^{2},
$$

$C$ is given by $C=4 c_{ \pm}^{2}\left[\left(c_{+}-c_{-}\right)\left(\mu \cdot a_{ \pm}\right)\right]^{-1}$, and we have defined the 1 -forms

$$
\begin{aligned}
\Omega(\rho) & =\mu \cdot \mathrm{d} \varphi+4 C \rho \frac{c_{+} c_{-}}{c_{+}-c_{-}} \mathrm{d} u \\
\omega_{I}(\rho) & =s_{I} \cdot \mathrm{d} \varphi+\frac{\rho}{2} C^{2}\left(s_{I} \cdot a_{+}+s_{I} \cdot a_{-}\right) \mathrm{d} u .
\end{aligned}
$$

We are also using the shorthand notations such as $s_{I i} a_{+}^{i}=s_{I} \cdot a_{+}$, or $\mu \cdot \mathrm{d} \varphi=\mu_{i} \mathrm{~d} \varphi^{i}$, etc. The parameters are subject to the constraints $\mu \cdot a_{ \pm} \neq 0$ and

$$
\frac{c_{+}^{2}}{\mu \cdot a_{+}}=\frac{c_{-}^{2}}{\mu \cdot a_{-}}, \quad \frac{c_{+}\left(s_{I} \cdot a_{+}\right)}{\mu \cdot a_{+}}=\frac{c_{-}\left(s_{I} \cdot a_{-}\right)}{\mu \cdot a_{-}}, \quad \pm 1=\left(c_{+}-c_{-}\right) \epsilon^{i j k \ldots m} s_{0 i} s_{1 j} s_{2 k} \cdots \mu_{m}
$$

but they are otherwise free. The coordinates $\varphi^{i}$ are $2 \pi$-periodic, $0 \leq \theta \leq \pi$, and $u, \rho$ are arbitrary. When writing " \pm ", we mean that the formulae hold for both signs.

Remarks 1) The number of free real parameters, after taking into account the constraints $(90)$, is $(D-3)(D-4)$. It is not clear that all near horizon geometries in the above theorem can be matched to known black hole solutions.

2) The possible horizon topologies for $B$ are encoded in the inter vectors $\underline{a}_{+}:=\underline{v}_{h-1}, \underline{a}_{-}:=$ $\underline{v}_{h+1} \in \mathbb{Z}^{D-3}$, see figure 2 .

3) A similar analysis for minimal supergravity in $D=5$ dimensions has been carried out, following the method of [70], in [95]. It would be interesting to carry out a corresponding analysis in 11-dimensional supergravity.

4) A similar result is not available at present for a non-zero cosmological constant, where the type of technique used in the proof does not seem to work.

\section{Other Theories in $D \geq 4$}

Our discussion of uniqueness theorems in both the rotating (stationary) and non-rotating (static) case has been restricted to the vacuum field equations, and to specific boundary conditions at infinity (and also to non-extremal black holes). Also, our discussion of some related other results, such as the sigma-model reduction, and Weyl-Papapetrou form, has been restricted to the vacuum theory. It is clearly of interest to (a) consider other theories, i.e., matter fields and (b) to discuss more general asymptotic boundary conditions. In this section, we give examples of what has been achieved in this direction, or what we think probably could be achieved without too much difficulty. The following list gives an indication of the situation. 


\begin{tabular}{c|c|c} 
Theorem & Theory & Boundary Conditions \\
\hline rigidity thm. & $(26)+$ null energy cond. & asympt. flat, (A)dS \\
topology thm. & dominant energy cond. & any \\
top. censorship & null conv. cond. & asympt. flat,KK,AdS(?) \\
staticity thm. & Einstein-Maxwell & asympt. flat \\
uniqueness stationary & vacuum $+U(1)^{D-3}$ symmetry & asympt. KK \\
& $\exists \sigma$-model formulation $+U(1)^{D-3}$ symmetry & asympt. KK \\
uniqueness static & Einstein-Maxwell-Dilaton & asympt. flat \\
& Einstein+sigma model & asympt. flat \\
& Einstein+form fields & asympt. flat
\end{tabular}

As is apparent from the list, general structural theorems such as the rigidity-, toplogy-, topological censorship theorems can be proved under fairly minimal assumptions, i.e. appropriate energy conditions, that are verified in a wide variety of matter models (however, extremal black holes often pose problems). On the other hand, the uniqueness theorems require more special input. The two main gaps in our understanding here are: 1) Stationary black holes in theories that do not admit a sigma-model reduction of the kind described above, either because (a) lack of symmetry or (b) due to the nature of the Lagrangian. This is a very significant shortcoming, because investigations based on approximations [27, 34] indicate that the required $U(1)^{D-3}$-symmetry is not generic, and because it excludes many theories of interest. 2) For static black holes, the uniqueness proofs work in much greater generality, but one does not know as yet e.g. how to treat asymptotic KK-boundary conditions, and again, extremal black holes are sometimes more difficult to deal with. In the following two subsections, we discuss some of these points in a bit more detail.

\subsection{Rotating stationary black holes}

Let us first indicate in which kinds of higher dimensional gravity theories, other than vacuum Einstein theory, and under what type of symmetry assumption, one might apply the uniqueness arguments presented in sec. 3.7 for rotating black holes. The bottleneck of the argument are the Weyl-Papapetrou form, orbit space theorem, and the existence of a divergence identity of the type of the Mazur- or Bunting identity, see sec. 3.6. The Weyl-Papapetrou form requires $D-2$ commuting Killing fields, together with the vanishing of the right side of eq. (46). This depends in general on the form of the matter stress-tensor, but is OK in general theories of the form (26), plus possibly Chern-Simons type terms. However, already this step does not work e.g. for non-abelian gauge fields. Both the Mazur- and Bunting identity are based on a sigma-model formulation of the dimensionally reduced theory. This also requires $D-2$ commuting Killing fields, but puts much more stringent further constraints. Examples of known sigma-model formulations for the dimensionally reduced theory to 3-dimensions (assuming $U(1)^{D-3}$ symmetry) are given in the following table: 


\begin{tabular}{c|c|c|c} 
Theory & $D$ & Sigma model? & Coset \\
\hline Einstein-Maxwell & 4 & yes & $S U(2,1) / S(U(1) \times U(2))[108]$ \\
Einstein-Maxwell & $\geq 5$ & no & - \\
Einstein-Yang-Mills & $\geq 4$ & no & - \\
Vacuum & $\geq 4$ & yes & $S L(D-2) / S O(D-2)[30,97]$ \\
Vacuum $+\Lambda$ & $\geq 4$ & no & - \\
Minimal Sugra & 5 & yes & $G_{2(+2)} / S O(4)[104,10]$ \\
Sugra & 11 & yes & $E_{8(+8)} / S O(16)[86,105]$ \\
Sugra & 10 & yes & $S O(8,8) / S O(8) \times S O(8))[86]$
\end{tabular}

Further models leading to sigma-models upon dimensional reduction are discussed in [16]. Note that most theories in the list which have a sigma-model formulation are related to supergravity theories. The coset formulation of the reduced Einstein-Maxwell system in $D=4$ is the basis of the classic uniqueness theorem for the charged Kerr black hole already described at the beginning. For Einstein-Maxwell theory in $D \geq 5$ dimensions, there is still a sigma-model formulation if one makes further, by-hand, restrictions on the form of the Maxwell field, and metric, see below.

$D=11$ Supergravity: Maybe to the most interesting case is the case of $D=11$ supergravity. This is based on the exceptional Lie-group ${ }^{13} E_{8(+8)}$ which has a -well-deservedreputation for being complicated. A parameterization of the coset was given e.g. by [105] (for original papers, see $[86,14]$ ); we outline how to get a uniqueness theorem parallel to that given in thm. 10. The details are in [71].

First, the bosonic part of the supergravity Lagrangian in 11 dimensions is

$$
S=\int_{\mathscr{M}} \frac{1}{2} R \star_{11} 1-F \wedge \star_{11} F-\frac{2}{3} B \wedge F \wedge F
$$

where $B$ is a 3 -form potential with field strength $F=\mathrm{d} B$. In parallel with the vacuum case, we assume 8 commuting axial Killing fields $\psi_{1}, \ldots, \psi_{8}$ in addition to the timelike Killing field $t$, i.e. an isometric action of $U(1)^{8} \times \mathbb{R}$ on the spacetime. Additionally, it is required that these vector fields Lie-derive also the potential $B$. Then it can be seen that the orbit space theorem still applies. Furthermore, the metric takes again the Weyl-Papapetrou form, because the right side of (46) vanishes. In particular, we can define the interval structure of the spacetime, with 8-dimensional vectors $\underline{v}_{J} \in \mathbb{Z}^{8}$. We can also define electric type charges by

$$
Q_{\mathrm{E}}[C]:=\int_{C}\left(\star_{11} F+B \wedge F\right)
$$

for any 7-cycle $C \in H_{7}(\mathscr{M}, \mathbb{Z})$. These provide further invariants that are not present in pure gravity. To define the coset, one first has to introduce the potentials analogous to (50). As in the vacuum case, we write the 11-dimensional metric $g$ as in (47), with 1-forms $A^{i}$, and

\footnotetext{
${ }^{13}$ The subscript $(+8)$ indicates a special real form.
} 
with $f_{i j}=g\left(\psi_{i}, \psi_{j}\right)$. Then, writing $B_{i}=i_{\psi_{i}} B, B_{i j}=i_{\psi_{i}} i_{\psi_{j}} B$ etc., one shows that scalar potentials $\varphi^{i j}, \chi_{i}$ can be defined by the following equations:

$$
\begin{aligned}
& F^{i j}-\mathrm{d} A^{k} B_{k}^{i j}=-\operatorname{det} f^{-1} \star_{3}\left(\mathrm{~d} \varphi^{i j}-\frac{1}{36} \mathrm{~d} B_{k l m} B_{n p q} \epsilon^{i j k l m n p q}\right) \\
& \mathrm{d} A_{k}=-\operatorname{det} f^{-1} \star_{3}\left(\mathrm{~d} \chi_{k}-B_{k i j} \mathrm{~d} \varphi^{i j}+\varphi^{i j} \mathrm{~d} B_{k i j}+\frac{1}{54} \epsilon^{i j l m n p q r} B_{i j k} \mathrm{~d} B_{l m n} B_{p q r}\right) .
\end{aligned}
$$

The effective 3-dimensional sigma-model Lagrangian for the model is then given by the same formula (52) as in pure gravity, but with a considerably more elaborate definition of $\Phi$ taking values in the coset $E_{8(+8)} / S O(16)$, which is defined in terms of the scalars $f_{i j}, \chi_{i}, B_{i j k}, \varphi^{i j}$. The precise definition is rather involved and was developed in [105]: The matrix $\Phi$ acts on 3 -tuples of tensors of the form ${ }^{14}\left(z^{M}{ }_{L}, x_{[L M N]}, y^{[L M N]}\right)$, where capital Roman letters run from $1, \ldots, 9$, where $z^{I}{ }_{I}=0$, and where a square bracket denotes anti-symmetrization. Define

$$
V_{I}^{J}=\left(\begin{array}{cc}
e_{i}{ }^{a} & -(\operatorname{det} f)^{\frac{1}{2}} \chi_{i} \\
0 & (\operatorname{det} f)^{\frac{1}{2}}
\end{array}\right)
$$

with $e_{i}{ }^{a} e_{k}{ }^{b} \delta_{a b}=f_{i k}$, and define

$$
\begin{aligned}
& v_{I J K}= \begin{cases}-2 \sqrt{3} B_{i j k} & \text { if } i=I, j=J, k=K \text { all } \leq 8 \\
0 & \text { otherwise. }\end{cases} \\
& w^{I J K}= \begin{cases}-6 \sqrt{3} \varphi^{i j} & \text { if } i=I, j=J \text { all } \leq 8 \text { and } K=9, \\
0 & \text { otherwise } .\end{cases}
\end{aligned}
$$

Next, define

$$
\begin{gathered}
Z= \\
\left(\begin{array}{ccc}
0 & \frac{1}{6}\left(w^{J[M N} \delta_{I}^{L]}-\frac{1}{9} w^{L M N} \delta_{I}^{J}\right) & -\frac{1}{6}\left(v_{I[M N} \delta_{L]}^{J}-\frac{1}{9} v_{L M N} \delta_{I}^{J}\right) \\
-3\left(v_{M[J K} \delta_{I]}^{L}-\frac{1}{9} v_{I J K} \delta_{M}^{L}\right) & 0 & \frac{1}{36 \sqrt{3}} \epsilon_{I L K P Q R L M N} w^{P Q R} \\
3\left(w^{L[J K} \delta_{M}^{I]}-\frac{1}{9} w^{I J K} \delta_{M}^{L}\right) & \frac{1}{36 \sqrt{3}} \epsilon^{I L K P Q R L M N} v_{P Q R} & 0
\end{array}\right)
\end{gathered}
$$

and set $\mathcal{V}_{-}=\exp Z$. Finally, define $\mathcal{V}_{+}$by

$$
\mathcal{V}_{+}=\left(\begin{array}{ccc}
V_{I}{ }^{A} V^{J}{ }_{B} & 0 & 0 \\
0 & V_{[I}{ }^{A} V_{J}^{B} V_{K]}{ }^{C} & 0 \\
0 & 0 & V^{[I}{ }_{A} V^{J}{ }_{B} V^{K]}{ }_{C}
\end{array}\right)
$$

where $V_{I}{ }^{A}$ and $V^{I}{ }_{A}$ are inverses of each other. Then $\Phi$ is given by

$$
\Phi=\mathcal{V} \cdot \tau(\mathcal{V})^{-1}, \quad \mathcal{V}=\mathcal{V}_{-} \mathcal{V}_{+}
$$

\footnotetext{
${ }^{14}$ This is the adjoint representation $\mathbf{2 4 8}$ of $E_{8(+8)}$, which under $S L(9)$ decomposes as $\mathbf{8 0} \oplus \mathbf{8 4} \oplus \overline{\mathbf{8 4}}$.
} 
where $\tau$ is the symmetric space involution corresponding to the involution on the Lie algebra $\mathfrak{e}_{8(+8)} \cong \mathfrak{s} o(16) \oplus \mathfrak{h}$ which acts by +1 on the first summand (the maximal abelian subalgebra) and by -1 on the second. With this definition of $\Phi$, the reduced action again takes precisely the same form (52), up to a different, irrelevant, numerical prefactor in front of the scalar field term.

One can now exploit divergence identities and prove, by the same general strategy as in the vacuum theory, that two non-extremal, single horizon, black hole solutions whose corresponding interval structure $\left\{\underline{v}_{J}, l_{J}\right\}$, angular momenta $J_{i}$, and charges $Q_{\mathrm{E}}[C]$ all coincide, must in fact be the same. To carry out these steps in detail, one must understand precisely how these data affect the boundary conditions on the fields $\Phi_{i}, i=1,2$ of the two solutions under consideration. For this in turn, one must understand how to describe all possible cycles $C \in H_{7}(\mathscr{M}, \mathbb{Z})$ in terms of the data $\left\{\underline{v}_{J}\right\}$, and how the information about the charges and angular momenta translates into information about the boundary conditions of the fields $\Phi_{i}$ for the two solutions under consideration. This is rather complicated indeed, but it can be done [71]. Apart from the technical complications related to the structure of $E_{8(+8)}$, the essential new feature compared to the vacuum theory are the charges $Q_{\mathrm{E}}[C]$ defined relative to the various cycles $C$, which now enter the proof. This issue arises also-but can be understood much more easily-in the case Einstein-Maxwell theory in 5 dimensions, so we will now explain it in that theory.

$D=5$ Einstein-Maxwell theory: As is seen from the above table, unlike minimal supergravity in $D=5$ dimensions, this theory actually does not have a sigma model formulation even in the presence of an isometry group $\mathbb{R} \times U(1)^{2}$ of the spacetime. However, it does have a sigma model formulation if we make a additional, by-hand, simplifying assumptions about the nature of the solutions to be considered. The simplifying assumptions are:

1. About the spacetime metric we assume that one of the axial Killing fields, say $\psi_{1}$, is orthogonal to the other Killing fields, $0=g\left(t, \psi_{1}\right)=g\left(\psi_{1}, \psi_{2}\right)$, and that it is hypersurface orthogonal, $\psi_{1} \wedge \mathrm{d} \psi_{1}=0$.

2. About the Maxwell field we assume that there is a 1-form $\xi$ orthogonal to the Killing fields such that $F=\frac{1}{2} \xi \wedge \psi_{1}$. It can easily be shown that, if the Maxwell field arises from a vector potential $F=\mathrm{d} A$ which is invariant under the Killing fields, then this will be the case if and only if $A$ is proportional to $\psi_{1}$ at each point in $M$. Note, however that we do not assume the existence of such a vector potential here.

Let us first point out the main simplifications which follow from assumptions 1) and 2). The first immediate consequence of 1 ) is that $J_{1}=0$. Secondly, because the Killing field $\psi_{1}$ is demanded to be orthogonal to $\psi_{2}$, if $v_{1} \psi_{1}+v_{2} \psi_{2}=0$ at a point in spacetime, then either $\underline{v}=\left(v_{1}, v_{2}\right)=(0,0)$, or $\underline{v}=(0,1),(1,0)$, or both axial Killing fields vanish. Thus, the interval structure (see sec. 3.4) of any solution satisfying assumption 1) can only be of the following possibilities (i) - (iv): 


\begin{tabular}{|c|c|c|}
\hline & Moduli $l_{J}$ & Vectors $\underline{v}_{J}$ \\
\hline (i) & $\infty, l_{1}, \ldots, l_{p}, \infty$ & $(1,0),(0,1), \ldots(1,0),(0,0),(1,0),(0,1) \ldots,(0,1)$ \\
\hline (ii) & $\infty, l_{1}, \ldots, l_{p}, \infty$ & $(1,0),(0,1), \ldots(0,1),(0,0),(0,1),(1,0) \ldots,(0,1)$ \\
\hline (iii) & $\infty, l_{1}, \ldots, l_{p}, \infty$ & $(1,0),(0,1), \ldots(0,1),(0,0),(1,0),(0,1) \ldots,(0,1)$ \\
\hline (iv) & $\infty, l_{1}, \ldots, l_{p}, \infty$ & $(1,0),(0,1), \ldots(1,0),(0,0),(0,1),(1,0) \ldots,(0,1)$ \\
\hline
\end{tabular}

Thus, the possible interval structures are severely restricted by 1 ). By table 2 , it then follows that the only possible horizon topologies are

$$
B \cong S^{1} \times S^{2} \quad \text { (black ring), } B \cong S^{3} \quad \text { (black hole) }
$$

with the first case realized when the vectors to the left and right of the horizon $\underline{v}_{h-1}, \underline{v}_{h+1}$ are equal [i.e., for the interval structures (i) and (ii)] and the second case realized when they are different [i.e., for the interval structures (iii) and (iv)]. In particular, the Lens-spaces $L(p, q)$ are excluded as possible horizon topologies by 1$)$.

From 2), the electric charge $Q_{\mathrm{E}}[C]=0$ associated with any 2-cycle $C$ vanishes, and the Maxwell field is completely characterized by the 1-form

$$
f=i_{\psi_{1}} F
$$

which is closed by the equations of motion for the Maxwell field, $\mathrm{d} f=0$. We define the twist 1-form by

$$
\omega=\frac{1}{2} \psi_{1} \wedge \psi_{2} \wedge \mathrm{d} \psi_{2}
$$

Again one can show that this is closed $d \omega=0$. It can then be shown that there exist globally defined potentials $\mathrm{d} \alpha=f, \mathrm{~d} \chi=\omega$. If the Maxwell field arises from a globally defined vector potential, $F=\mathrm{d} A$ - which we do not assume - then $\alpha=i_{\psi_{1}} A$.

Using the potentials $\alpha, \chi$, one can now write down the reduced Einstein-Maxwell equations on the orbit space $\hat{M}$, in the form of a pair of sigma model equations [75]: Let $\nu, w, u$ be the functions on $\hat{\mathscr{M}}$ defined through:

$$
e^{2 u}=g\left(\psi_{1}, \psi_{1}\right), \quad e^{-u+2 w}=g\left(\psi_{2}, \psi_{2}\right), \quad e^{-u+2 w+2 \nu}=g(\mathrm{~d} r, \mathrm{~d} r),
$$

with $r$ defined as in eq. (43). Then the complete Einstein-Maxwell equations are equivalent to the following pair of matrix equations on the upper complex half plane $\hat{\mathscr{M}}=\{(r, z) \mid r>0\}$ :

$$
\begin{aligned}
& \mathrm{d} \star_{2}\left(r \Phi_{1}^{-1} \mathrm{~d} \Phi_{1}\right)=0, \\
& \mathrm{~d} \star_{2}\left(r \Phi_{2}^{-1} \mathrm{~d} \Phi_{2}\right)=0,
\end{aligned}
$$

together with an equation for $\nu$, which we do not write down. The matrix fields are defined in terms of $u, w, \alpha, \chi$ by

$$
\Phi_{1}=\left(\begin{array}{cc}
e^{u}+\frac{1}{3} e^{-u} \alpha^{2} & \frac{1}{\sqrt{3}} e^{-u} \alpha \\
\frac{1}{\sqrt{3}} e^{-u} \alpha & e^{-u}
\end{array}\right)
$$


and

$$
\Phi_{2}=\left(\begin{array}{cc}
e^{2 w}+4 \chi^{2} e^{-2 w} & 2 \chi e^{-2 w} \\
2 \chi e^{-2 w} & e^{-2 w}
\end{array}\right)
$$

The first two equations state that the matrix fields $\Phi_{1}$ and $\Phi_{2}$ each satisfy the equations of a 2-dimensional sigma-model. The matrix fields are real, symmetric, positive definite, with determinant equal to 1 on the interior of $\hat{\mathscr{M}}$, so we have as target space two decoupled copies of $S L(2) / S O(2)$. With the aid of this sigma model formulation, one proves [75]:

Theorem 15. Consider two stationary, asymptotically flat, Einstein-Maxwell black hole spacetime of dimension 5, having one time-translation Killing field and two axial Killing fields. We also assume that there are no points with discrete isotropy subgroup under the action of the isometry group in the exterior of the black hole, and we assume that the Killing and Maxwell fields satisfy the assumptions 1) and 2) above. If the two solutions have the same interval structures, the same values of the mass $m$, same angular momentum $J_{2}$, and same magnetic charges $Q_{\mathrm{M}}\left[C_{l}\right]=(2 \pi)^{-1} \int_{C_{l}} F$ for all 2-cycles $C_{l} \in H_{2}(\mathscr{M}, \mathbb{Z})$, then they are isometric.

The proof is very similar to that in the vacuum case, except for the following new consideration: To prove the boundedness of the quantities analogous to $\sigma$ in (62) built from the matrices $\Phi_{1}$ for two given solutions as in the theorem, one also needs to use that

$$
\alpha(z)-\alpha\left(z^{\prime}\right)=\frac{1}{2 \pi} \int_{C} F=\frac{1}{2 \pi} Q_{\mathrm{M}}[C] .
$$

where $z, z^{\prime}$ are from two boundary intervals labeled by the same integer vectors $(1,0)$ or $(0,1)$, for a suitable cycle $C \in H_{2}(\mathscr{M})$. Knowing that the magnetic charges of both solutions are the same then enables one via this identity to show that boundary value $(r=0)$ of the $\alpha$ 's for both solutions are also the same. An argument of this sort-but rather more complicated-also works in 11-dimensional supergravity.

The proof shows that the non-trivial 2-cycles [i.e., basis elements of $H_{2}(\mathscr{M})$ ] in the exterior of the spacetime may in fact be obtained as follows. We know that the real axis $\{r=0\}$ bounding $\hat{\mathscr{M}}=\{(r, z) \mid r>0\}$ is divided into intervals, each labeled with an integer 2-vector $\underline{v}_{J}=(1,0)$ or $\underline{v}_{J}=(0,1)$, see the above table. Now consider all possible curves $\hat{\gamma}_{p}, p=1,2, \ldots$ in $\hat{\mathscr{M}}$ with the property that $\hat{\gamma}_{p}$ starts at $z$ on an interval labeled $(1,0)$, and ends at $z^{\prime}$ on another interval labeled $(1,0)$, with no interval with label $(1,0)$ in between. If we now lift $\hat{\gamma}_{p}$ to a curve $\gamma_{p}$ in $\mathscr{M}$, and act with all isometries generated by $\psi_{1}$ on the image of this curve, then we generate a closed 2 -surface $C_{p}$ in $\mathscr{M}$ which is topologically a 2 -sphere for all $p$, see the following picture. 


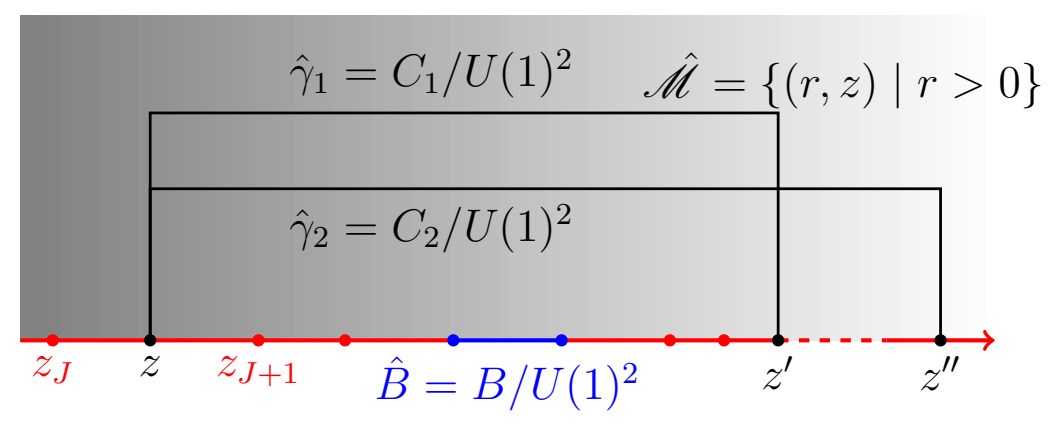

We may repeat this by replacing $\hat{\gamma}_{p}, p=1,2, \ldots$ with a set of curves each starting on an interval labeled $(0,1)$, and ending on another interval labeled $(0,1)$, with no interval with label $(0,1)$ in between. If we again lift these curves to curves in $\mathscr{M}$, and act with all isometries generated by $\psi_{2}$, then we generate a set of topologically inequivalent closed 2-surfaces $\tilde{C}_{q}, q=1,2, \ldots$ in $\mathscr{M}$, each of which is topologically a 2 -sphere. It may be seen that the set of 2-surfaces $\left\{C_{p}, \tilde{C}_{q}\right\}$ forms a basis of $H_{2}(\mathscr{M})$, and also of $H_{2}(\Sigma)$, where the 4-manifold $\Sigma$ is a spatial slice going from infinity to the horizon (so that topologically $\mathscr{M}=\mathbb{R} \times \Sigma)$. In this 4-manifold, we can compute intersection numbers as $C_{p}: \tilde{C}_{q}= \pm 1$ or $=0$, depending on whether the corresponding curves in $\hat{\mathscr{M}}$ intersect or not. The rank of $H_{2}(\Sigma)=H_{2}(\mathscr{M})$ in the cases (i) through (iv) in the above table, and the intersection matrix $Q_{\Sigma}: H_{2}(\Sigma) \times H_{2}(\Sigma) \rightarrow \mathbb{Z}$ is therefore easily computed to be given by

$$
Q_{\Sigma}=\bigoplus^{m}\left(\begin{array}{ll}
0 & 1 \\
1 & 0
\end{array}\right)
$$

where $m$ is related to the number of intervals in the interval structure (compare eq. 1). Only the magnetic charges $Q_{\mathrm{M}}\left[C_{p}\right]$ enter in the proof of the above theorem. The magnetic charges $Q_{\mathrm{M}}\left[\tilde{C}_{q}\right]$ are not needed and in fact vanish, due to assumptions 1) and 2) at the beginning of this section. Thus, for the simplest interval structure $(0,1),(0,0),(1,0)$, there are no nontrivial magnetic charges, and the unique solution within the class studied here is completely specified by $J_{2}, m$. In fact, this unique solution is the Myers-Perry black hole [107], with vanishing Maxwell field.

\subsection{Static black holes}

To keep the discussion simple, we have considered above uniqueness theorems for nonextremal static black holes only within vacuum Einstein gravity, and for asymptotically flat boundary conditions. There are also uniqueness theorems for other theories, and other boundary conditions (although not for KK-type asymptotic conditions in as far as we are aware), as well as for extremal black holes in Einstein-Maxwell theory. We summarize some results in this direction. 
1. Dilatonic black holes: In higher dimensions, we have the exact solutions of dilatonic Einstein-Maxwell theory [55]. One can prove their uniqueness in the same manner here [53]. The same technique also applies to the case of Einstein coupled to sigma-model fields [120].

2. Einstein-form fields: A uniqueness theorem Einstein- $p$-form fields when $p \geqslant(D+1) / 2$ was obtained in [31]. In the proof of this theorem, one uses the same kind of conformal transformation employed in the proof of the Schwarzschild-Tangherlini spacetime above in sec. 3.8 .

3. Extremal black holes in Einstein-Maxwell theory: As already mentioned, in the extremal limit, there are exact solutions for charged multi-black holes. The uniqueness of such solutions has been proven $[24,121]$. See also [122] for related work.

4. Asymptotically de Sitter/anti-de Sitter: There are exact static solutions of Einsteingravity + cosmological constant, the Schwarzschild-(anti-)de Sitter solutions. In $[11,2]$ attempts have been made to generalize the static uniqueness to the asymptotically anti-de Sitter case, but the situation remains open. In particular, recent numerical investigations [9] indicate that AdS-spacetime is unstable to perturbations, and the end state of the dynamical evolution of an instability may well be an unknown, nonstandard black hole spacetime. See also Ref. [89] for perturbative investigations of potential new families of static black holes in these theories.

\subsection{Supersymmetric black holes}

An entirely different approach to the classification of black hole spacetimes, applicable to supergravity theories, is to consider solutions with Killing spinors, rather than Killing vectors. Unlike the Killing vector condition, $\mathscr{L}_{t} g=0$, which takes the same form in any theory, the precise form of the Killing spinor condition depends on the theory under consideration. In 4 dimensional $N=2$ supergravity, this analysis has been completed first by [129], but the method is applicable, in principle, in any dimension in which supergravity theories can exist (i.e. $D \leq 11$ ). Consider for example the case of minimal supergravity in $D=5$ dimensions, with action ${ }^{15}$

$$
S=\int_{\mathscr{M}} \frac{1}{2} R \star_{5} 1+F \wedge \star_{5} F+\frac{4}{3 \sqrt{3}} F \wedge F \wedge A
$$

where $A$ is a one-form with field strength $F=\mathrm{d} A$. The above action must be supplemented in the full supergravity theory by terms involving fermionic partner fields with half integral spin. But at the classical level, it does not make much physical sense to consider solutions in which the fermionic fields are non-trivial, and one thus sets them to zero. The full action with superpartners is invariant under the odd supersymmetry transformation $\delta_{\epsilon}$ depending

\footnotetext{
${ }^{15}$ In this section, the signature is $(+----)$, opposite from the rest of the paper.
} 
linearly on a symplectic Majorana spinor field ${ }^{16} \epsilon^{a}, a=1,2$, which maps the bosonic fields $g, A$ to a combination of fields depending on the fermionic superpartners, and which maps the fermionic superpartners to an expression involving the bosonic fields. Let us demand that a purely bosonic field configuration be "supersymmetric", in the sense that it is annihilated by $\delta_{\epsilon}$. Since the fermionic fields vanish by assumption, we automatically have $\delta_{\epsilon} g=0=\delta_{\epsilon} A$, whereas the condition that $\delta_{\epsilon}$ on the fermionic fields (spin 3/2-partner of the metric) gives zero amounts to ${ }^{17}$

$$
\left(\nabla_{\mu}+\frac{1}{4 \sqrt{3}}\left(\gamma_{\mu \nu \sigma}+g_{\mu \nu} \gamma_{\sigma}\right) F^{\nu \sigma}\right) \epsilon^{a}=0
$$

This is the Killing-spinor condition for $D=5$ minimal supergravity. The existence of a Killing spinor is very restrictive-much more so than demanding merely the existence of a Killing vector. This can be seen e.g. from the fact that, since a supersymmetry transformation "squares to an infinitesimal translation" (i.e. a Lie-derivative), the "square" of a Killing-spinor automatically has to be a Killing vector, and also a symmetry of the other bosonic fields in the theory, i.e. $F$ in our case. More precisely, define

$$
t^{\mu}:=\frac{1}{2} \epsilon_{a b} \bar{\epsilon}^{a} \gamma^{\mu} \epsilon^{b}
$$

where $\epsilon^{a b}$ is the standard 2-dimensional symplectic matrix. Then $t$ is necessarily a time-like, or null, Killing vector field, $\mathscr{L}_{t} g=0$, and one also necessarily has $\mathscr{L}_{t} F=0$. If $(\mathscr{M}, g, A)$ represents an asymptotically flat black hole spacetime, then $t$ is by construction tangent to the generators of the horizon, and because it is timelike or null both inside and outside the horizon, and null on the horizon, we must have $\mathrm{d}(g(t, t))=0$ on the horizon. Using the standard formula for the surface gravity $\mathrm{d}(g(t, t))=-2 \kappa t$ then shows that $\kappa=0$, i.e. the black hole is necessarily extremal. In fact, the existence of a Killing spinor is even more stringent than the above argument suggests, because it implies many more differential relations than just the Killing vector equation. These have been exploited systematically by [48] (and previously by Tod [129] in the case of $N=2$ supergravity in $D=4$ ). [48] proceed by defining the real tensorial quantities $f, X_{1}, X_{2}, X_{3}$ by

$$
f \epsilon^{a b}=\bar{\epsilon}^{a} \epsilon^{b}, \quad\left(\begin{array}{cc}
X_{1}+i X_{2} & i X_{3} \\
-i X_{3} & X_{1}-i X_{2}
\end{array}\right)^{a b}=\bar{\epsilon}^{a} \gamma_{\mu \nu} \epsilon^{b} \mathrm{~d} x^{\mu} \wedge \mathrm{d} x^{\nu} .
$$

The field equations and Killing spinor equation then imply, among other things, the following further relations:

$$
f^{2}=g(t, t), \quad \mathrm{d} f=-\frac{2}{\sqrt{3}} i_{t} F, \quad \mathrm{~d} X_{i}=0, \quad \mathrm{~d} \star_{5} X_{i}=-\frac{2}{\sqrt{3}} F \wedge X_{i} .
$$

\footnotetext{
${ }^{16}$ This is a pair of 4-dimensional complex spinor fields $\epsilon^{a}, a=1,2$, subject to the condition $\bar{\epsilon}^{a}=\left(\epsilon^{a}\right)^{T} C$, with $C$ the charge conjugation endomorphism of the Clifford algebra defined by $C\left(\gamma_{\mu}\right)^{T} C^{-1}=\gamma_{\mu}$. The conjugate spinor is defined by $\bar{\epsilon}^{a} \delta_{a b}=\left(\epsilon^{a}\right)^{*} \beta \epsilon_{a b}$, where $\beta$ is the endomorphism of the Clifford algebra defined by $\beta\left(\gamma_{\mu}\right)^{*} \beta^{-1}=\gamma_{\mu}$, and where $*$ is hermitian adjoint.

${ }^{17} \gamma_{\mu_{1} \ldots \mu_{n}}=\gamma_{\left[\mu_{1}\right.} \cdots \gamma_{\left.\mu_{n}\right]}$ denote the generators of the Clifford algebra $\operatorname{Cliff}(T \mathscr{M})$.
} 
It turns out that, starting from these equations, one can locally determine [48] all possible analytic field configurations with Killing spinor, which can then be analytically continued. In this way, one can obtain, in principle, a classification (which includes black holes, but also other types of spacetimes) of supersymmetric bosonic configurations $(g, A)$. We think that it would be worthwhile to understand in detail the global structure of these solutions. Progress towards this goal has been made in the special case of spherical horizons by [118].

A similar analysis can presumably be carried out in other, more complicated, supergravity theories. Of particular interest would be a similarly complete classification of solutions with various numbers of independent Killing spinors in 11-dimensional supergravity. Progress in this direction has been made e.g. in the papers [50, 49], see also references therein.

Thus, in supersymmetric theories, the classification of black hole solutions via Killingspinors seems a feasible-albeit complicated-task. However, one cannot hope to obtain in this way solutions at non-zero temperature, i.e. non-extremal ones.

\section{Summary and open issues}

In this review, we have tried to give an overview about what is known about black hole uniqueness theorems for higher dimensional, stationary, black hole spacetimes. Along the way, we have described several related general structural results about such black holes such as the topology, rigidity, and staticity theorems, which are also of independent interest. These arguments involve a considerable breadth of mathematical methods, from differential geometry, to topology, to group theory, to ergodic theory, and to PDE theory, which are combined in a non-trivial fashion. As we have indicated, the state of knowledge concerning higher dimensional black holes in general, and uniqueness theorems in particular, is much less satisfactory than in four dimensions, although also in four dimensions there are still many open issues for many theories other than Einstein-Maxwell theory. In general, it seems that our knowledge about static or supersymmetric black holes in higher dimensions is more advanced than for stationary solutions, but there are also still important open issues for static and supersymmetric black holes. As open issues that in our opinion deserve attention we would like to mention:

1. To obtain uniqueness theorems for stationary higher dimensional black holes, one needs to assume more symmetries than seem to be generic on the basis of the investigations [34, 27], and one is at the moment restricted to models in which the dimensionally reduced theories can be cast into the framework of sigma-models with negatively curved target spaces. There are some ideas how to replace the interval structure that seems important in existing theorems [60, 68], but more ideas are needed.

2. To obtain uniqueness theorems for static black holes, one is for the moment restricted to asymptotically flat boundary conditions, excluding thus e.g. the, very interesting and relevant, Kaluza-Klein type boundary conditions. 
3. It would also be important to achieve a full classicfication of supersymmetric black holes in more complicated supergravity theories than have been analyzed so far, in particular in 11-dimensional supergravity.

We feel that input from numerical methods will be needed to get a more complete picture of the landscape of higher dimensional black holes, and to help answer some of these questions in particular.

\section{Acknowledgments}

This work is supported in part (AI) by the Grant-in-Aid for Scientific Research Fund of the JSPS (C)No. 22540299. We would like to thank the referee for his careful reading of this manuscript.

\section{References}

[1] Andersson, L., Mars, M. and Simon, W.: Local existence of dynamical and trapping horizons. Phys. Rev. Lett. 95 (2005) 111102 [gr-qc/0506013].

[2] Anderson, M.T., Chruściel, P.T., and Delay, E.: Nontrivial, static, geodesically complete, vacuum space-times with a negative cosmological constant. JHEP 0210063 (2002)

[3] Amsel, A.J., Horowitz, G.T., Marolf, D., and Roberts, M.M.: Uniqueness of Extremal Kerr and Kerr-Newman Black Holes. Phys. Rev. D81 024033 (2010)

[4] Atiyah, M. and Hitchin, N.: The geometry and dynamics of magnetic monopoles, Princeton University Press, 1988.

[5] Atiyah, M.F., Hitchin, N.J. and Singer, I.M.: Self-duality in four-dimensional Riemannian geometry. Proc. Roy. Soc. London A362 (1978), 425461.

[6] Bardeen, J.M., Carter, B., and Hawking, S.W.: The Four laws of black hole mechanics. Commun. Math. Phys. 31 161-170 (1973)

[7] Bardeen, J.M. and Horowitz, G.T.: The Extreme Kerr throat geometry: A Vacuum analog of AdS(2) x S**2. Phys. Rev. D60 104030 (1999)

[8] Belinski, V. and Verdaguer, E.: Gravitational solitons. Cambridge, UK: Univ. Pr. (2001) $258 \mathrm{p}$

[9] Bizon, P. and Rostworowski, A.: On weakly turbulent instability of anti-de Sitter space. Phys. Rev. Lett. 107, 031102 (2011) [arXiv:1104.3702 [gr-qc]]. 
[10] Bouchareb, A., Clement, G., Chen, C.-M., Gal'tsov, D.V., Scherbluk, N.G., and Wolf, T.: $\mathrm{G}(2)$ generating technique for minimal $\mathrm{D}=5$ supergravity and black rings. Phys. Rev. D 76, 104032 (2007) [Erratum-ibid. D 78, 029901 (2008)] [arXiv:0708.2361 [hep-th]].

[11] Boucher, W., Gibbons, G.W. and Horowitz, G.T.: A Uniqueness Theorem For Anti-De Sitter Space-Time, Phys. Rev. D 30 (1984), 2447.

[12] Bunting, G. L.: Proof of the uniqueness conjecture for black holes. (PhD Thesis, Univ. of New England, Armidale, N.S.W., 1983)

[13] Bunting, G.L. and Masood-ul-Alam, A.K.M.: Gen. Rel. Grav. 19147 (1987)

[14] Breitenlohner, P. and Maison, D, Ann. H. Poincare 46, 216 (1987)

[15] Breitenlohner, P. and Maison, D.: On the Geroch Group. Annales Poincare Phys. Theor. 46 (1987) 215.

[16] Breitenlohner, P., Maison, D., and Gibbons, G.W.: Four-Dimensional Black Holes from Kaluza-Klein Theories. Commun. Math. Phys. 120, 295 (1988).

[17] Carter, B.: Axisymmetric black hole has only two degrees of freedom. Phys. Rev. Lett. 26, 331-333 (1971)

[18] Chen, Y. and Teo, E.: Black holes on gravitational instantons. Nucl. Phys. B 850, 253 (2011) [arXiv:1011.6464 [hep-th]].

[19] Chruściel, P.T.: The classification of static vacuum spacetimes containing an asymptotically flat spacelike hypersurface with compact interior. Class. Quant. Grav. 16, 661-687 (1999)

[20] Chruściel, P. T. and Costa, J.L.: On uniqueness of stationary vacuum black holes. arXiv:0806.0016 [gr-qc].

[21] Chruściel, P. T., Costa, J.L., and Heusler, M.: Stationary Black Holes: Uniqueness and Beyond. Living Rev. Rel. 15 (2012) 7

[22] Chruściel, P. T., Galloway, G. J. and Solis, D.: Topological censorship for Kaluza-Klein space-times. Annales Henri Poincare 10 893-912 (2009)

[23] Chruściel, P.T. and Nguyen, Luc.: A uniqueness theorem for degenerate Kerr-Newman black holes. Annales Henri Poincare 11 585-609 (2010)

[24] Chruściel, P.T., Reall, H.S., and Tod, P.: On non-existence of static vacuum black holes with degenerate components of the event horizon. Class. Quant. Grav. 23 (2006), 549 [arXiv:gr-qc/0512041]. 
[25] Chruściel, P.T., and Tod, P.: The classification of static electro-vacuum space-times containing an asymptotically flat spacelike hypersurface with compact interior. Commun. Math. Phys. 271, 577-589 (2007)

[26] Dafermos, M. and Rodnianski, I.: The Red-shift effect and radiation decay on black hole spacetimes. Commun. Pure Appl. Math. 62 (2009) 859 [gr-qc/0512119].

[27] Dias, O.J.C., Horowitz, G.T., and Santos, J.E.: Gravitational Turbulent Instability of Anti-de Sitter Space. arXiv:1109.1825 [hep-th].

[28] Dias, O.J.C., Figueras, P., Monteiro, R., Reall, H.S., and Santos, J.E.: An instability of higher-dimensional rotating black holes. JHEP 1005, 076 (2010) [arXiv:1001.4527 [hep-th]];

[29] Durkee, M. and Reall, H.S.: Perturbations of near-horizon geometries and instabilities of Myers-Perry black holes. Phys. Rev. D 83, 104044 (2011) [arXiv:1012.4805 [hep-th]].

[30] Ehlers, J. Les Theories Relativistes de la Gravitation, CNRS preprint (1959)

[31] Emparan, R., Ohashi, S. and Shiromizu, T.: No-dipole-hair theorem for higherdimensional static black holes. Phys. Rev. D 82 (2010), 084032 [arXiv:1007.3847 [hepth]].

[32] Emparan, R. and Reall, H.S.: A rotating black ring in five dimensions. Phys. Rev. Lett. 88, 101101 (2002)

[33] Emparan, R. and Reall, H.S.: Black Holes in Higher Dimensions. Living Rev. Rel. 11, $6(2008)$

[34] Emparan, R., Harmark, T., Niarchos, V., and Obers, N.A.: Essentials of Blackfold Dynamics. JHEP 1003, 063 (2010) [arXiv:0910.1601 [hep-th]]; Camps, J. and Emparan, R.: Derivation of the blackfold effective theory. arXiv:1201.3506 [hep-th].

[35] Elvang, H. and Figueras, P.: Black saturn. JHEP 0705050 (2007)

[36] Figueras, P., Kunduri, H.K., Lucietti, J., and Rangamani, M.: Extremal vacuum black holes in higher dimensions. Phys. Rev. D78, 044042 (2008)

[37] Figueras, P. and Lucietti, J.: On the uniqueness of extremal vacuum black holes. Class. Quant. Grav. 27095001 (2010)

[38] Figueras, P., Lucietti, J., and Wiseman, T.: Ricci solitons, Ricci flow, and strongly coupled CFT in the Schwarzschild Unruh or Boulware vacua. Class. Quant. Grav. 28 $215018(2011)$ 
[39] Figueras, P., and Wiseman, T.: Gravity and large black holes in Randall-Sundrum II braneworlds. [arXiv:1105.2558 [hep-th]].

[40] Figueras, P. Murata, K., and Reall, H.S.: Black hole instabilities and local Penrose inequalities. Class. Quant. Grav. 28, 225030 (2011) [arXiv:1107.5785 [gr-qc]].

[41] Fintushel, R.: Classification of Circle Actions on 4-Manifolds. Trans. AMS 242377 (1978)

[42] Freedman, M.H.: The topology of four-dimensional manifolds. J. Diff. Geom. 17357453 (1982)

[43] Friedman, J.L., Schleich, K. and Witt, D.M.: Topological Censorship. Phys. Rev. Lett. 711486 (1993)

[44] Friedrich, H., Racz, I. and Wald, R.M.: On the rigidity theorem for spacetimes with a stationary event horizon or a compact Cauchy horizon. Commun. Math. Phys. 204, 691-707 (1999)

[45] Galloway, G.J., Schleich, K., Witt, D.M., and Woolgar, E.: Topological censorship and higher genus black holes. Phys. Rev. D 60, 104039 (1999)

[46] Galloway, G.J. and Schoen, R.: A generalization of Hawking's black hole topology theorem to higher dimensions. Commun. Math. Phys. 266, 571 (2006)

[47] Galloway, G.J.: Rigidity of marginally trapped surfaces and the topology of black holes. Commun. Anal. Geom. 16, 217 (2008)

[48] Gauntlett, J.P., Gutowski, J.B., Hull, C.M., Pakis, S. and Reall, H.S.: All supersymmetric solutions of minimal supergravity in five- dimensions. Class. Quant. Grav. 20, 4587 (2003) [hep-th/0209114].

[49] Gauntlett, J.P., Gutowski, J.B., and Pakis, S.: The Geometry of $D=11$ null Killing spinors. JHEP 0312, 049 (2003) [hep-th/0311112].

[50] Gauntlett, J.P. and Pakis, S.: The Geometry of $D=11$ killing spinors. JHEP 0304, 039 (2003) [hep-th/0212008].

[51] Geroch, R.P.: A Method for generating new solutions of Einstein's equation. 2. J. Math. Phys. 13, 394 (1972).

[52] Gibbons G.W., Ida, D., and Shiromizu, T.: Uniqueness and non-uniqueness of static black holes in higher dimensions. Phys. Rev. Lett. 89, 041101 (2002) 
[53] Gibbons G.W., Ida, D., and Shiromizu, T.: Uniqueness of (dilatonic) charged black holes and black p-branes in higher dimensions. Phys. Rev. D 66, 044010 (2002) [arXiv:hep-th/0206136]

[54] Gibbons, G.W., Ida, D., and Shiromizu, T.: Uniqueness and non-uniqueness of static vacuum black holes in higher dimensions. Prog. Theor. Phys. Suppl. 148, 284 (2002) [arXiv:gr-qc/0203004].

[55] Gibbons, G.W. and Maeda, K.-i.: Black Holes And Membranes In Higher Dimensional Theories With Dilaton Fields. Nucl. Phys. B 298741 (1988)

[56] Gregory, R. and Laflamme, R.: Black strings and p-branes are unstable. Phys. Rev. Lett. 70, 2837 (1993) [hep-th/9301052].

[57] Gibbons, G.W., Hawking, S.W., Horowitz, G.T., and Perry, M.J.: Positive Mass Theorems For Black Holes. Commun. Math. Phys. 88, 295 (1983).

[58] Hajicek, P.: Three remarks on axisymmetric stationary horizons. Commun. Math. Phys. 36, 305-320 (1974)

[59] Harmark, T.: Stationary and axisymmetric solutions of higher-dimensional general relativity. Phys. Rev. D 70, 124002 (2004)

[60] Harmark, T.: Domain structure of black hole space-times. Phys. Rev. D 80, 024019 (2009)

[61] Harmark, T. and Olesen, P.: Structure of stationary and axisymmetric metrics. Phys. Rev. D 72, 124017 (2005)

[62] Hawking, S.W.: Black holes in general relativity. Commun. Math. Phys. 25, 152-166 (1972)

[63] Hawking, S.W. and Ellis, G.F.R.: The large scale structure of space-time Cambridge: Cambridge University Press, 1973

[64] Helfgott, C., Oz, Y., and Yanay, Y.: On the topology of black hole event horizons in higher dimensions JHEP 0602, 025 (2006)

[65] Helgason, S.: "Differential Geometry on Symmetric Spaces," Academic Press 2000

[66] Heusler, M.: Black hole uniqueness theorems Cambridge University Press, Cambridge, (1996)

[67] Hollands, S., Ishibashi, A., and Marolf, D.: Comparison between various notions of conserved charges in asymptotically AdS-spacetimes. Class. Quant. Grav. 22, 2881 (2005) [hep-th/0503045]. 
[68] Hollands, S., Holland, J. and Ishibashi, A.: Further Restrictions on the Topology of Stationary Black Holes in Five Dimensions. arXiv:1002.0490

[69] Hollands, S. and Ishibashi, A.: On the 'Stationary Implies Axisymmetric' Theorem for Extremal Black Holes in Higher Dimensions. Commun. Math. Phys. 291, 403-441 (2009)

[70] Hollands, S. and Ishibashi, A.: All vacuum near horizon geometries in arbitrary dimensions. Annales Henri Poincaré 10, 1537 (2010)

[71] Hollands, S.: Black hole uniqueness theorems and new thermodynamic identities in eleven dimensional supergravity. arXiv:1204.3421 [gr-qc].

[72] Hollands, S., Ishibashi, A. and Wald, R.M.: A Higher dimensional stationary rotating black hole must be axisymmetric. Commun. Math. Phys. 271, 699-722 (2007)

[73] Hollands, S. and Yazadjiev, S.: Uniqueness Theorem for 5-Dimentional Black Holes with Two Axial Killing Fields. Commun. Math. Phys. 283, 749-768 (2008)

[74] Hollands, S. and Yazadjiev, S.: A Uniqueness theorem for stationary Kaluza-Klein black holes. Commun. Math. Phys. 302 (2011) 631 [arXiv:0812.3036 [gr-qc]].

[75] Hollands, S. and Yazadjiev, S.: A Uniqueness theorem for 5-dimensional EinsteinMaxwell black holes. Class. Quant. Grav. 25 (2008) 095010 [arXiv:0711.1722 [gr-qc]].

[76] Hollands, S. and Wald, R.M.: Stability of Black Holes and Black Branes. arXiv:1201.0463 [gr-qc]

[77] Hwang, S.: Rigidity Theorem for Ricci Flat Metrics. Geometriae Dedicata 71 5-17 (1998)

[78] Ida, D., Ishibashi, A., and Shiromizu, T.: Topology and Uniqueness of Higher Dimensional Black Holes. Prog. Theor. Phys. Suppl. 189 52-92 (2011)

[79] Iguchi, H. and Mishima, T.: Black diring and infinite nonuniqueness. Phys. Rev. D 75 $064018(2007)$

[80] Ishibashi, A. and Kodama, H.: Stability of higher-dimensional Schwarzschild black holes. Prog. Theor. Phys. 110, 901-919 (2003)

[81] Izumi, K.: Orthogonal black di-ring solution. Prog. Theor. Phys. 119, 757 (2008) [arXiv:0712.0902 [hep-th]].

[82] Ionescu, A.D. and Klainerman, S.: Uniqueness Result for Ill-Posed Characteristic Problems in Curved Space-Times. Commun. Math. Phys. 285, 875-900 (2009) 
[83] Ionescu, A.D. and Klainerman, S.: On the uniqueness of smooth, stationary black holes in vaccum Invent. Math. 175, 35-102 (2009)

[84] Israel, W.: Event horizons in static vacuum space-times. Phys. Rev., 164, 1776-1779 (1967)

[85] Jost, J.: "Riemannian Geometry and Geometric Analysis," Springer 2002

[86] Julia, B.: "Group Disintegrations," Nuffield Gravity Workshop, Cambridge (1980)

[87] Kerr, R.P.: Gravitational field of a spinning mass as an example of algebraically special metrics. Phys. Rev. Lett. 11 237-238 (1963)

[88] Kobayashi, S. and Nomizu, K.: Foundations of Differential Geometry (Interscience Publishers, New York, 1969), Vo. II, Sec. VII, Theorem 5.1.

[89] Kodama, H.: Perturbative uniqueness of black holes near the static limit in arbitrary dimensions. Prog. Theor. Phys. 112 (2004) 249 [hep-th/0403239].

[90] Kodama, H. and Ishibashi, A.: A master equation for gravitational perturbations of maximally symmetric black holes in higher dimensions. Prog. Theor. Phys. 110, $701-722(2003)$

[91] Kunduri, H.K., Lucietti, J., and Reall, H.S.: Near-horizon symmetries of extremal black holes. Class. Quant. Grav. 24, 4169 (2007)

[92] Kunduri, H.K. and Lucietti, J.: A Classification of near-horizon geometries of extremal vacuum black holes. J. Math. Phys. 50082502 (2009)

[93] Kunduri, H.K. and Lucietti, J.: Static near-horizon geometries in five dimensions. Class. Quant. Grav. 26 (2009), 245010.

[94] H. K. Kunduri and J. Lucietti, "An infinite class of extremal horizons in higher dimensions," Commun. Math. Phys. 303, 31 (2011) [arXiv:1002.4656 [hep-th]].

[95] H. K. Kunduri and J. Lucietti, "Constructing near-horizon geometries in supergravities with hidden symmetry," JHEP 1107, 107 (2011) [arXiv:1104.2260 [hep-th]].

[96] Lewandowski, J. and Pawlowski, T.: Extremal isolated horizons: A Local uniqueness theorem. Class. Quant. Grav. 20 587-606 (2003)

[97] Maison, D.: Ehlers-Harrison-Type Transformations for Jordan's Extended Theory of Gravitation. Gen. Relativ. Gravit. 10, 717 (1979)

[98] Mars, M.: A Space-time characterization of the Kerr metric. Class. Quant. Grav. 16, 2507 (1999) [gr-qc/9904070], Simon, W.: Gen. Relativ. Grav. 16465 (1984) 
[99] M. Mars, "Stability of MOTS in totally geodesic null horizons," Class. Quant. Grav. 29, 145019 (2012) [arXiv:1205.1724 [gr-qc]].

[100] Masood-ul-Alam, A.K.M.: Uniqueness proof of static charged dilaton black hole. Class. Quant. Grav. 10 2649-2656 (1993)

[101] Mazur, P.O.: A global identity for nonlinear $\sigma$-models. J. Phys. A, 100, 341-344 (1984)

[102] Moncrief, V. and Isenberg, J.: Symmetries of cosmological Cauchy horizons. Commun. Math. Phys. 89, 387-413 (1983)

[103] Moncrief, V. and Isenberg, J.: Symmetries of Higher-Dimensional Black Holes. Class. Quantum Grav. 25, 195015 (2008)

[104] Mizoguchi, S. and Ohta, N.: More on the similarity between D = 5 simple supergravity and M theory. Phys. Lett. B 441, 123 (1998) [hep-th/9807111].

[105] Mizoguchi, S. 'y.: E(10) symmetry in one-dimensional supergravity. Nucl. Phys. B 528, 238 (1998) [hep-th/9703160].

[106] Morisawa, Y. and Ida, D.: A boundary value problem for the five-dimensional stationary rotating black holes. Phys. Rev. D 69, 124005 (2004)

[107] Myers, R.C. and Perry, M.J.: Black holes in higher dimensional space-times. Annals Phys. 172, 304 (1986)

[108] Kramer, D. and Neugebauer, G.: An exact stationary solution of the einstein-maxwell equation. (in german). Annalen Phys. 24, 59 (1969).

[109] Newman, E.T., Couch, E., Chinnapared, K., Exton, A., Prakash, A., and Torrence, R.: Metric of a Rotating Charged Mass. J. Math. Phys., 6, 918-919 (1965)

[110] Orlik, P. and Raymond, F.: Actions of the torus on 4-manifolds I. Transactions of the AMS 152, (1972)

[111] Orlik, P. and Raymond, F.: Actions of the torus on 4-manifolds II. Topology 13 89-112 (1974)

[112] Penrose, R.: "Techniques of Differential Topology in Relativity" Regional Conference Series in Applied Math. 7, SIAM, Philadelphia (1972)

[113] Pomeransky, A.A. and Sen'kov, R.A.: Black ring with two angular momenta. [arXiv: hep-th/0612005]

[114] Racz, I.: On further generalization of the rigidity theorem for spacetimes with a stationary event horizon or a compact Cauchy horizon. Class. Quant. Grav. 17, 153 (2000) 
[115] Racz, I.: A simple proof of the recent generalizations of Hawking's black hole topology theorem. Class. Quant. Grav. 25, 162001 (2008)

[116] Racz, I. and Wald, R.M.: Extensions of spacetimes with Killing horizons. Class. Quantum Grav. 9, 2643-2656 (1992)

[117] Racz, I. and Wald, R.M.: Global extensions of spacetimes describing asymptotic final states of black holes. Class. Quantum Grav. 13, 539-552 (1996)

[118] H. S. Reall, "Higher dimensional black holes and supersymmetry," Phys. Rev. D 68 (2003) 024024 [Erratum-ibid. D 70 (2004) 089902] [hep-th/0211290].

[119] Robinson, D.C.: Uniqueness of the Kerr black hole. Phys. Rev. Lett. 34, 905-906 (1975)

[120] Rogatko, M.: Uniqueness theorem for static black hole solutions of sigma models in higher dimensions. Class. Quant. Grav. 19, L151 (2002)

[121] Rogatko, M.: Uniqueness theorem of static degenerate and non-degenerate charged black holes in higher dimensions. Phys. Rev. D 67, 084025 (2003)

[122] Rogatko, M.: Staticity theorem for higher dimensional generalized Einstein-Maxwell system. Phys. Rev. D 71, 024031 (2005)

[123] Rogatko, M.: Extrema of mass, first law of black hole mechanics and staticity theorem in Einstein-Maxwell axion dilaton gravity. Phys. Rev. D 58, 044011 (1998) [hepth/9807012].

[124] Ruback, P.: A new uniqueness theorem for charged black holes. Class. Quant. Grav. 5, L155-L159 (1988)

[125] Schoen, R. and Yau, S.T.: On the Proof of the positive mass conjecture in general relativity. Commun. Math. Phys. 65(1979), 45; R. Schoen, in Topics in calculus of variations (Montecatini Terme, 1987), Lecture Notes in Mathematics Vol. 1365 (Springer, New York,1989).

[126] Sudarsky, D. and Wald, R.M.: Extrema of mass, stationarity, and staticity, and solutions to the Einstein Yang-Mills equations. Phys. Rev. D 46, 1453-1474 (1992)

[127] Tangherlini, F.R.: Schwarzschild field in n dimensions and the dimensionality of space problem. Nuovo Cim. 27636 (1963)

[128] Teukolsky, S.A.: Rotating Black Holes: Separable Wave Equations for Gravitational and Electromagnetic Perturbations. Phys. Rev. Lett. 291114 (1972)

[129] Tod, K.P.: All Metrics Admitting Supercovariantly Constant Spinors. Phys. Lett. B 121, 241 (1983). 
[130] Wald, R.M.: General Relativity. Chicago: University of Chicago Press (1984)

[131] Wald, R.M.: The Thermodynamics of Black Holes. Living Reviews in Relativity 4, 2001-6 (2001) [gr-qc/9912119]

[132] Walters, P.: An Introduction to Ergodic Theory. Springer-Verlag New York, 1982

[133] Weinstein, G.: On the Dirichlet problem for harmonic maps with prescribed singularities. Duke Math. J. 77 (1995) No.1, 135-165

[134] Witten, E.: A Simple Proof Of The Positive Energy Theorem. Commun. Math. Phys. 80381 (1981)

[135] Zerilli, F.J.: Effective potential for even parity Regge-Wheeler gravitational perturbation equations. Phys. Rev. Lett. 24, 737 (1970). 